\title{
6. NSW Hospitals
}

\section{Onset and augmentation of labour}

Table 33 shows onset or augmentation of labour for Local Health Districts, and Table 34 for individual hospitals by

maternity service level where at least 200 mothers gave birth in 2009.

Table 33. Onset and augmentation of labour by Local Health District of hospital, NSW 2009

\begin{tabular}{|c|c|c|c|c|c|c|c|c|c|c|c|c|c|c|c|c|c|c|}
\hline \multirow{3}{*}{$\begin{array}{l}\text { Local Health } \\
\text { District }\end{array}$} & \multicolumn{18}{|c|}{ Onset and augmentation of labour } \\
\hline & \multicolumn{2}{|c|}{ Spontaneous } & \multicolumn{2}{|c|}{$\begin{array}{l}\text { Spontaneous } \\
\text { augmented } \\
\text { with ARM }\end{array}$} & \multicolumn{2}{|c|}{$\begin{array}{l}\text { Spontaneous } \\
\text { augmented } \\
\text { with oxytocics/ } \\
\text { prostagland\# }\end{array}$} & \multicolumn{2}{|c|}{ No labour } & \multicolumn{2}{|c|}{$\begin{array}{l}\text { Induced } \\
\text { oxytocics/ } \\
\text { prosta- } \\
\text { glandins }\end{array}$} & \multicolumn{2}{|c|}{$\begin{array}{c}\text { Induced } \\
- \text { ARM only }\end{array}$} & \multicolumn{2}{|c|}{$\begin{array}{c}\text { Induced - } \\
\text { ARM + } \\
\text { oxytocics/ } \\
\text { prostaglandins }\end{array}$} & \multicolumn{2}{|c|}{$\begin{array}{l}\text { Induced } \\
\text { - other\#\# }\end{array}$} & \multicolumn{2}{|c|}{ TOTAL } \\
\hline & No. & $\%$ & No. & $\%$ & No. & $\%$ & No. & $\%$ & No. & $\%$ & No. & $\%$ & No. & $\%$ & No. & $\%$ & No. & $\%$ \\
\hline Sydney & 3042 & 45.3 & 131 & 2.0 & 1079 & 16.1 & 1075 & 16.0 & 570 & 8.5 & 84 & 1.3 & 730 & 10.9 & 4 & 0.1 & 6715 & 100.0 \\
\hline $\begin{array}{l}\text { South Western } \\
\text { Sydney }\end{array}$ & 4620 & 44.6 & 1003 & 9.7 & 1003 & 9.7 & 1471 & 14.2 & 885 & 8.5 & 110 & 1.1 & 1230 & 11.9 & 35 & 0.3 & 10357 & 100.0 \\
\hline $\begin{array}{l}\text { South Eastern } \\
\text { Sydney }\end{array}$ & 3427 & 43.5 & 546 & 6.9 & 715 & 9.1 & 1175 & 14.9 & 812 & 10.3 & 129 & 1.6 & 1055 & 13.4 & 14 & 0.2 & 7873 & 100.0 \\
\hline $\begin{array}{l}\text { Illawarra } \\
\text { Shoalhaven }\end{array}$ & 1414 & 42.9 & 243 & 7.4 & 202 & 6.1 & 424 & 12.9 & 298 & 9.0 & 84 & 2.6 & 622 & 18.9 & 6 & 0.2 & 3293 & 100.0 \\
\hline Western Sydney & 3811 & 41.8 & 632 & 6.9 & 774 & 8.5 & 1271 & 13.9 & 980 & 10.7 & 101 & 1.1 & 1550 & 17.0 & 5 & 0.1 & 9124 & 100.0 \\
\hline $\begin{array}{l}\text { Nepean Blue } \\
\text { Mountains }\end{array}$ & 2219 & 45.6 & 299 & 6.1 & 242 & 5.0 & 781 & 16.0 & 447 & 9.2 & 85 & 1.7 & 790 & 16.2 & 8 & 0.2 & 4871 & 100.0 \\
\hline Northern Sydney & 1997 & 41.6 & 306 & 6.4 & 499 & 10.4 & 754 & 15.7 & 506 & 10.5 & 85 & 1.8 & 651 & 13.6 & 1 & 0.0 & 4799 & 100.0 \\
\hline Central Coast & 1191 & 42.5 & 145 & 5.2 & 253 & 9.0 & 492 & 17.6 & 289 & 10.3 & 39 & 1.4 & 390 & 13.9 & 3 & 0.1 & 2802 & 100.0 \\
\hline $\begin{array}{l}\text { Hunter New } \\
\text { England }\end{array}$ & 4242 & 48.4 & 377 & 4.3 & 559 & 6.4 & 1094 & 12.5 & 940 & 10.7 & 217 & 2.5 & 1284 & 14.7 & 44 & 0.5 & 8757 & 100.0 \\
\hline Northern NSW & 1795 & 48.4 & 342 & 9.2 & 313 & 8.4 & 492 & 13.3 & 357 & 9.6 & 61 & 1.6 & 342 & 9.2 & 10 & 0.3 & 3712 & 100.0 \\
\hline Mid North Coast & 978 & 44.1 & 286 & 12.9 & 190 & 8.6 & 310 & 14.0 & 190 & 8.6 & 45 & 2.0 & 217 & 9.8 & 3 & 0.1 & 2219 & 100.0 \\
\hline Southern NSW & 719 & 51.0 & 154 & 10.9 & 112 & 7.9 & 177 & 12.5 & 126 & 8.9 & 26 & 1.8 & 97 & 6.9 & 0 & 0.0 & 1411 & 100.0 \\
\hline Murrumbidgee & 937 & 47.4 & 183 & 9.3 & 95 & 4.8 & 306 & 15.5 & 222 & 11.2 & 64 & 3.2 & 167 & 8.4 & 3 & 0.2 & 1977 & 100.0 \\
\hline Western NSW & 1490 & 43.6 & 403 & 11.8 & 213 & 6.2 & 511 & 15.0 & 251 & 7.3 & 107 & 3.1 & 437 & 12.8 & 4 & 0.1 & 3416 & 100.0 \\
\hline Far West & 119 & 50.0 & 21 & 8.8 & 17 & 7.1 & 36 & 15.1 & 35 & 14.7 & 2 & 0.8 & 8 & 3.4 & 0 & 0.0 & 238 & 100.0 \\
\hline Private Hospitals & 6533 & 28.0 & 1767 & 7.6 & 2116 & 9.1 & 6280 & 26.9 & 1772 & 7.6 & 471 & 2.0 & 4334 & 18.6 & 41 & 0.2 & 23314 & 100.0 \\
\hline TOTAL* & 38697 & 40.7 & 6840 & 7.2 & 8382 & 8.8 & 16649 & 17.5 & 8680 & 9.1 & 1710 & 1.8 & 13904 & 14.6 & 181 & 0.2 & 95043 & 100.0 \\
\hline
\end{tabular}


Table 34. Onset and augmentation of labour by hospital and maternity service level, NSW 2009\#

\begin{tabular}{|c|c|c|c|c|c|c|c|c|c|c|c|c|c|c|c|c|c|c|}
\hline \multirow{3}{*}{$\begin{array}{l}\text { Maternity service level- } \\
\text { hospital }\end{array}$} & \multicolumn{18}{|c|}{ Onset and augmentation of labour } \\
\hline & \multicolumn{2}{|c|}{ Spontaneous } & \multicolumn{2}{|c|}{$\begin{array}{l}\text { Spontaneous } \\
\text { augmented } \\
\text { with ARM }\end{array}$} & \multicolumn{2}{|c|}{$\begin{array}{l}\text { Spontaneous } \\
\text { augmented } \\
\text { with } \\
\text { oxytocics/ } \\
\text { prosta- } \\
\text { glandins\#\# }\end{array}$} & \multicolumn{2}{|c|}{ No labour } & \multicolumn{2}{|c|}{$\begin{array}{l}\text { Induced } \\
\text { oxytocics/ } \\
\text { prosta- } \\
\text { glandins }\end{array}$} & \multicolumn{2}{|c|}{$\begin{array}{c}\text { Induced } \\
\text { - ARM only }\end{array}$} & \multicolumn{2}{|c|}{$\begin{array}{l}\text { Induced - } \\
\text { ARM + } \\
\text { oxytocics/ } \\
\text { prosta- } \\
\text { glandins }\end{array}$} & \multicolumn{2}{|c|}{$\begin{array}{l}\text { Induced } \\
\text { - other\#\#\# }\end{array}$} & \multicolumn{2}{|c|}{ TOTAL } \\
\hline & No. & $\%$ & No. & $\%$ & No. & $\%$ & No. & $\%$ & No. & $\%$ & No. & $\%$ & No. & $\%$ & No. & $\%$ & No. & $\%$ \\
\hline \multicolumn{19}{|l|}{ Level 6} \\
\hline Royal Prince Alfred & 2249 & 44.8 & 0 & 0.0 & 841 & 16.7 & 845 & 16.8 & 447 & 8.9 & 73 & 1.5 & 566 & 11.3 & 1 & 0.0 & 5022 & 100.0 \\
\hline Royal North Shore & 748 & 36.2 & 133 & 6.4 & 247 & 12.0 & 376 & 18.2 & 211 & 10.2 & 32 & 1.5 & 319 & 15.4 & 0 & 0.0 & 2066 & 100.0 \\
\hline Royal Hospital for Women & 1757 & 43.0 & 301 & 7.4 & 293 & 7.2 & 712 & 17.4 & 408 & 10.0 & 83 & 2.0 & 522 & 12.8 & 11 & 0.3 & 4087 & 100.0 \\
\hline Liverpool & 1328 & 43.6 & 312 & 10.2 & 308 & 10.1 & 472 & 15.5 & 261 & 8.6 & 27 & 0.9 & 330 & 10.8 & 10 & 0.3 & 3048 & 100.0 \\
\hline Nepean & 1648 & 46.1 & 218 & 6.1 & 182 & 5.1 & 573 & 16.0 & 304 & 8.5 & 47 & 1.3 & 596 & 16.7 & 8 & 0.2 & 3576 & 100.0 \\
\hline Westmead & 1695 & 37.0 & 367 & 8.0 & 431 & 9.4 & 734 & 16.0 & 533 & 11.6 & 58 & 1.3 & 768 & 16.7 & 1 & 0.0 & 4587 & 100.0 \\
\hline John Hunter & 1751 & 47.2 & 163 & 4.4 & 296 & 8.0 & 413 & 11.1 & 343 & 9.2 & 88 & 2.4 & 627 & 16.9 & 30 & 0.8 & 3711 & 100.0 \\
\hline TOTAL & 11176 & 42.8 & 1494 & 5.7 & 2598 & 10.0 & 4125 & 15.8 & 2507 & 9.6 & 408 & 1.6 & 3728 & 14.3 & 61 & 0.2 & 26097 & 100.0 \\
\hline Level 5 & & & & & & & & & & & & & & & & & & \\
\hline Gosford & 978 & 38.1 & 125 & 4.9 & 253 & 9.9 & 492 & 19.2 & 289 & 11.3 & 38 & 1.5 & 390 & 15.2 & 3 & 0.1 & 2568 & 100.0 \\
\hline St. George & 1073 & 42.9 & 158 & 6.3 & 282 & 11.3 & 309 & 12.3 & 290 & 11.6 & 26 & 1.0 & 364 & 14.5 & 2 & 0.1 & 2504 & 100.0 \\
\hline Blacktown & 1443 & 45.1 & 173 & 5.4 & 207 & 6.5 & 399 & 12.5 & 307 & 9.6 & 36 & 1.1 & 628 & 19.6 & 4 & 0.1 & 3197 & 100.0 \\
\hline Lismore Base & 670 & 50.8 & 95 & 7.2 & 79 & 6.0 & 202 & 15.3 & 135 & 10.2 & 26 & 2.0 & 108 & 8.2 & 4 & 0.3 & 1319 & 100.0 \\
\hline Tweed Heads & 637 & 42.6 & 150 & 10.0 & 177 & 11.8 & 172 & 11.5 & 124 & 8.3 & 31 & 2.1 & 200 & 13.4 & 4 & 0.3 & 1495 & 100.0 \\
\hline Port Macquarie Base & 369 & 47.1 & 66 & 8.4 & 80 & 10.2 & 101 & 12.9 & 67 & 8.6 & 7 & 0.9 & 93 & 11.9 & 0 & 0.0 & 783 & 100.0 \\
\hline Wollongong & 965 & 41.4 & 180 & 7.7 & 156 & 6.7 & 256 & 11.0 & 203 & 8.7 & 51 & 2.2 & 512 & 22.0 & 6 & 0.3 & 2329 & 100.0 \\
\hline Maitland & 736 & 48.2 & 43 & 2.8 & 106 & 6.9 & 214 & 14.0 & 189 & 12.4 & 23 & 1.5 & 209 & 13.7 & 6 & 0.4 & 1526 & 100.0 \\
\hline Wagga Wagga Base & 381 & 50.4 & 38 & 5.0 & 30 & 4.0 & 121 & 16.0 & 77 & 10.2 & 21 & 2.8 & 86 & 11.4 & 2 & 0.3 & 756 & 100.0 \\
\hline TOTAL & 7252 & 44.0 & 1028 & 6.2 & 1370 & 8.3 & 2266 & 13.8 & 1681 & 10.2 & 259 & 1.6 & 2590 & 15.7 & 31 & 0.2 & 16477 & 100.0 \\
\hline Level 4 & & & & & & & & & & & & & & & & & & \\
\hline Canterbury & 793 & 46.8 & 131 & 7.7 & 238 & 14.1 & 230 & 13.6 & 123 & 7.3 & 11 & 0.6 & 164 & 9.7 & 3 & 0.2 & 1693 & 100.0 \\
\hline Hornsby & 526 & 45.4 & 81 & 7.0 & 109 & 9.4 & 158 & 13.6 & 116 & 10.0 & 21 & 1.8 & 146 & 12.6 & 1 & 0.1 & 1158 & 100.0 \\
\hline Manly & 480 & 42.1 & 67 & 5.9 & 132 & 11.6 & 166 & 14.6 & 135 & 11.9 & 25 & 2.2 & 134 & 11.8 & 0 & 0.0 & 1139 & 100.0 \\
\hline Mona Vale & 138 & 43.8 & 10 & 3.2 & 11 & 3.5 & 54 & 17.1 & 44 & 14.0 & 6 & 1.9 & 52 & 16.5 & 0 & 0.0 & 315 & 100.0 \\
\hline Sutherland & 597 & 46.6 & 87 & 6.8 & 140 & 10.9 & 154 & 12.0 & 114 & 8.9 & 20 & 1.6 & 169 & 13.2 & 1 & 0.1 & 1282 & 100.0 \\
\hline Auburn & 673 & 50.2 & 92 & 6.9 & 136 & 10.1 & 138 & 10.3 & 140 & 10.4 & 7 & 0.5 & 154 & 11.5 & 0 & 0.0 & 1340 & 100.0 \\
\hline Fairfield & 896 & 47.6 & 232 & 12.3 & 216 & 11.5 & 224 & 11.9 & 120 & 6.4 & 7 & 0.4 & 179 & 9.5 & 7 & 0.4 & 1881 & 100.0 \\
\hline Campbelltown & 1232 & 45.7 & 237 & 8.8 & 167 & 6.2 & 420 & 15.6 & 260 & 9.7 & 47 & 1.7 & 317 & 11.8 & 13 & 0.5 & 2693 & 100.0 \\
\hline Bankstown-Lidcombe & 931 & 42.9 & 167 & 7.7 & 243 & 11.2 & 274 & 12.6 & 205 & 9.4 & 22 & 1.0 & 324 & 14.9 & 5 & 0.2 & 2171 & 100.0 \\
\hline Hawkesbury & 386 & 44.8 & 45 & 5.2 & 28 & 3.3 & 132 & 15.3 & 98 & 11.4 & 31 & 3.6 & 141 & 16.4 & 0 & 0.0 & 861 & 100.0 \\
\hline Coffs Harbour & 414 & 38.4 & 171 & 15.8 & 94 & 8.7 & 180 & 16.7 & 78 & 7.2 & 32 & 3.0 & 107 & 9.9 & 3 & 0.3 & 1079 & 100.0 \\
\hline Grafton Base & 199 & 40.4 & 51 & 10.4 & 50 & 10.2 & 70 & 14.2 & 88 & 17.9 & 1 & 0.2 & 31 & 6.3 & 2 & 0.4 & 492 & 100.0 \\
\hline Tamworth Base & 367 & 44.0 & 41 & 4.9 & 52 & 6.2 & 120 & 14.4 & 101 & 12.1 & 13 & 1.6 & 134 & 16.0 & 7 & 0.8 & 835 & 100.0 \\
\hline Manning Base & 383 & 50.9 & 34 & 4.5 & 25 & 3.3 & 108 & 14.3 & 84 & 11.2 & 27 & 3.6 & 92 & 12.2 & 0 & 0.0 & 753 & 100.0 \\
\hline Dubbo Base & 454 & 37.9 & 185 & 15.4 & 90 & 7.5 & 163 & 13.6 & 68 & 5.7 & 51 & 4.3 & 188 & 15.7 & 0 & 0.0 & 1199 & 100.0 \\
\hline Orange Base & 324 & 36.5 & 96 & 10.8 & 41 & 4.6 & 169 & 19.1 & 58 & 6.5 & 36 & 4.1 & 160 & 18.0 & 3 & 0.3 & 887 & 100.0 \\
\hline Goulburn Base & 127 & 42.1 & 34 & 11.3 & 60 & 19.9 & 44 & 14.6 & 20 & 6.6 & 0 & 0.0 & 17 & 5.6 & 0 & 0.0 & 302 & 100.0 \\
\hline Shoalhaven & 415 & 47.5 & 56 & 6.4 & 38 & 4.4 & 160 & 18.3 & 75 & 8.6 & 30 & 3.4 & 99 & 11.3 & 0 & 0.0 & 873 & 100.0 \\
\hline Griffith Base & 206 & 50.2 & 38 & 9.3 & 26 & 6.3 & 58 & 14.1 & 38 & 9.3 & 22 & 5.4 & 22 & 5.4 & 0 & 0.0 & 410 & 100.0 \\
\hline TOTAL & 9541 & 44.7 & 1855 & 8.7 & 1896 & 8.9 & 3022 & 14.1 & 1965 & 9.2 & 409 & 1.9 & 2630 & 12.3 & 45 & 0.2 & 21363 & 100.0 \\
\hline Level 3 & & & & & & & & & & & & & & & & & & \\
\hline Blue Mountains & 121 & 49.4 & 14 & 5.7 & 10 & 4.1 & 45 & 18.4 & 17 & 6.9 & 6 & 2.4 & 32 & 13.1 & 0 & 0.0 & 245 & 100.0 \\
\hline Kempsey & 153 & 50.0 & 40 & 13.1 & 16 & 5.2 & 29 & 9.5 & 45 & 14.7 & 6 & 2.0 & 17 & 5.6 & 0 & 0.0 & 306 & 100.0 \\
\hline Murwillumbah & 119 & 57.2 & 18 & 8.7 & 7 & 3.4 & 48 & 23.1 & 10 & 4.8 & 3 & 1.4 & 3 & 1.4 & 0 & 0.0 & 208 & 100.0 \\
\hline Armidale & 232 & 53.0 & 16 & 3.7 & 16 & 3.7 & 60 & 13.7 & 61 & 13.9 & 19 & 4.3 & 33 & 7.5 & 1 & 0.2 & 438 & 100.0 \\
\hline Inverell & 51 & 20.0 & 3 & 1.2 & 27 & 10.6 & 62 & 24.3 & 55 & 21.6 & 4 & 1.6 & 53 & 20.8 & 0 & 0.0 & 255 & 100.0 \\
\hline Mudgee & 120 & 52.9 & 15 & 6.6 & 14 & 6.2 & 32 & 14.1 & 24 & 10.6 & 3 & 1.3 & 19 & 8.4 & 0 & 0.0 & 227 & 100.0 \\
\hline Bathurst Base & 307 & 54.8 & 53 & 9.5 & 35 & 6.3 & 77 & 13.8 & 55 & 9.8 & 7 & 1.3 & 25 & 4.5 & 1 & 0.2 & 560 & 100.0 \\
\hline Bega & 152 & 54.1 & 37 & 13.2 & 14 & 5.0 & 24 & 8.5 & 27 & 9.6 & 11 & 3.9 & 16 & 5.7 & 0 & 0.0 & 281 & 100.0 \\
\hline Moruya & 154 & 47.4 & 32 & 9.8 & 13 & 4.0 & 57 & 17.5 & 38 & 11.7 & 12 & 3.7 & 19 & 5.8 & 0 & 0.0 & 325 & 100.0 \\
\hline Queanbeyan & 180 & 58.3 & 37 & 12.0 & 14 & 4.5 & 28 & 9.1 & 29 & 9.4 & 3 & 1.0 & 18 & 5.8 & 0 & 0.0 & 309 & 100.0 \\
\hline Bowral & 232 & 41.2 & 55 & 9.8 & 69 & 12.3 & 81 & 14.4 & 39 & 6.9 & 7 & 1.2 & 80 & 14.2 & 0 & 0.0 & 563 & 100.0 \\
\hline
\end{tabular}


Table 34. (Continued)

\begin{tabular}{|c|c|c|c|c|c|c|c|c|c|c|c|c|c|c|c|c|c|c|}
\hline \multirow{3}{*}{$\begin{array}{l}\text { Maternity service level- } \\
\text { hospital }\end{array}$} & \multicolumn{18}{|c|}{ Onset and augmentation of labour } \\
\hline & \multicolumn{2}{|c|}{ Spontaneous } & \multicolumn{2}{|c|}{$\begin{array}{l}\text { Spontaneous } \\
\text { augmented } \\
\text { with ARM }\end{array}$} & \multicolumn{2}{|c|}{$\begin{array}{l}\text { Spontaneous } \\
\text { augmented } \\
\text { with } \\
\text { oxytocics/ } \\
\text { prosta- } \\
\text { glandins\#\# }\end{array}$} & \multicolumn{2}{|c|}{ No labour } & \multicolumn{2}{|c|}{$\begin{array}{l}\text { Induced } \\
\text { oxytocics/ } \\
\text { prosta- } \\
\text { glandins }\end{array}$} & \multicolumn{2}{|c|}{$\begin{array}{l}\text { Induced } \\
\text { - ARM only }\end{array}$} & \multicolumn{2}{|c|}{$\begin{array}{l}\text { Induced - } \\
\text { ARM + } \\
\text { oxytocics/ } \\
\text { prosta- } \\
\text { glandins }\end{array}$} & \multicolumn{2}{|c|}{$\begin{array}{l}\text { Induced } \\
\text { - other\#\#\# }\end{array}$} & \multicolumn{2}{|c|}{ TOTAL } \\
\hline & No. & $\%$ & No. & $\%$ & No. & $\%$ & No. & $\%$ & No. & $\%$ & No. & $\%$ & No. & $\%$ & No. & $\%$ & No. & $\%$ \\
\hline Broken Hill Base & 119 & 50.0 & 21 & 8.8 & 17 & 7.1 & 36 & 15.1 & 35 & 14.7 & 2 & 0.8 & 8 & 3.4 & 0 & 0.0 & 238 & 100.0 \\
\hline Other hospitals & 1237 & 48.7 & 264 & 10.4 & 131 & 5.2 & 342 & 13.5 & 267 & 10.5 & 60 & 2.4 & 238 & 9.4 & 1 & 0.0 & 2540 & 100.0 \\
\hline TOTAL & 3177 & 48.9 & 605 & 9.3 & 383 & 5.9 & 921 & 14.2 & 702 & 10.8 & 143 & 2.2 & 561 & 8.6 & 3 & 0.0 & 6495 & 100.0 \\
\hline \multicolumn{19}{|l|}{ Level 2} \\
\hline Wyong & 213 & 91.0 & 20 & 8.5 & 0 & 0.0 & 0 & 0.0 & 0 & 0.0 & 1 & 0.4 & 0 & 0.0 & 0 & 0.0 & 234 & 100.0 \\
\hline Other hospitals & 603 & 70.6 & 65 & 7.6 & 18 & 2.1 & 35 & 4.1 & 53 & 6.2 & 19 & 2.2 & 61 & 7.1 & 0 & 0.0 & 854 & 100.0 \\
\hline TOTAL & 816 & 75.0 & 85 & 7.8 & 18 & 1.7 & 35 & 3.2 & 53 & 4.9 & 20 & 1.8 & 61 & 5.6 & 0 & 0.0 & 1088 & 100.0 \\
\hline \multicolumn{19}{|l|}{ Private } \\
\hline Mater, North Sydney & 728 & 29.1 & 198 & 7.9 & 185 & 7.4 & 826 & 33.1 & 211 & 8.4 & 83 & 3.3 & 265 & 10.6 & 3 & 0.1 & 2499 & 100.0 \\
\hline North Shore Private & 634 & 24.1 & 224 & 8.5 & 321 & 12.2 & 794 & 30.2 & 123 & 4.7 & 85 & 3.2 & 435 & 16.6 & 10 & 0.4 & 2626 & 100.0 \\
\hline Sydney Adventist & 1106 & 49.2 & 0 & 0.0 & 0 & 0.0 & 496 & 22.1 & 124 & 5.5 & 26 & 1.2 & 494 & 22.0 & 2 & 0.1 & 2248 & 100.0 \\
\hline North Gosford Private & 194 & 23.2 & 46 & 5.5 & 66 & 7.9 & 287 & 34.3 & 81 & 9.7 & 20 & 2.4 & 141 & 16.9 & 1 & 0.1 & 836 & 100.0 \\
\hline Hurstville Private & 239 & 24.8 & 82 & 8.5 & 137 & 14.2 & 244 & 25.3 & 93 & 9.7 & 11 & 1.1 & 156 & 16.2 & 1 & 0.1 & 963 & 100.0 \\
\hline Kareena Private & 129 & 18.9 & 30 & 4.4 & 55 & 8.1 & 241 & 35.3 & 34 & 5.0 & 11 & 1.6 & 182 & 26.6 & 1 & 0.1 & 683 & 100.0 \\
\hline St. George Private & 410 & 22.6 & 148 & 8.1 & 237 & 13.0 & 491 & 27.0 & 158 & 8.7 & 33 & 1.8 & 336 & 18.5 & 4 & 0.2 & 1817 & 100.0 \\
\hline Prince of Wales Private & 554 & 24.4 & 282 & 12.4 & 198 & 8.7 & 708 & 31.2 & 201 & 8.9 & 49 & 2.2 & 275 & 12.1 & 1 & 0.0 & 2268 & 100.0 \\
\hline Norwest Private & 269 & 22.0 & 100 & 8.2 & 137 & 11.2 & 201 & 16.4 & 76 & 6.2 & 9 & 0.7 & 431 & 35.2 & 2 & 0.2 & 1225 & 100.0 \\
\hline Sydney Southwest Private & 301 & 28.5 & 60 & 5.7 & 124 & 11.7 & 238 & 22.5 & 97 & 9.2 & 26 & 2.5 & 209 & 19.8 & 2 & 0.2 & 1057 & 100.0 \\
\hline Nepean Private & 269 & 28.9 & 74 & 7.9 & 50 & 5.4 & 237 & 25.4 & 101 & 10.8 & 14 & 1.5 & 186 & 20.0 & 1 & 0.1 & 932 & 100.0 \\
\hline Westmead Private & 545 & 23.0 & 295 & 12.5 & 343 & 14.5 & 540 & 22.8 & 198 & 8.4 & 23 & 1.0 & 419 & 17.7 & 5 & 0.2 & 2368 & 100.0 \\
\hline Figtree Private & 174 & 17.2 & 80 & 7.9 & 88 & 8.7 & 258 & 25.5 & 65 & 6.4 & 35 & 3.5 & 310 & 30.6 & 3 & 0.3 & 1013 & 100.0 \\
\hline Newcastle Private & 683 & 36.3 & 96 & 5.1 & 108 & 5.7 & 451 & 24.0 & 85 & 4.5 & 36 & 1.9 & 419 & 22.3 & 5 & 0.3 & 1883 & 100.0 \\
\hline Calvary, Wagga Wagga & 235 & 37.5 & 28 & 4.5 & 41 & 6.5 & 175 & 27.9 & 102 & 16.3 & 9 & 1.4 & 37 & 5.9 & 0 & 0.0 & 627 & 100.0 \\
\hline Other hospitals & 63 & 23.4 & 24 & 8.9 & 26 & 9.7 & 93 & 34.6 & 23 & 8.6 & 1 & 0.4 & 39 & 14.5 & 0 & 0.0 & 269 & 100.0 \\
\hline TOTAL & 6533 & 28.0 & 1767 & 7.6 & 2116 & 9.1 & 6280 & 26.9 & 1772 & 7.6 & 471 & 2.0 & 4334 & 18.6 & 41 & 0.2 & 23314 & 100.0 \\
\hline TOTAL NSW** & 38697 & 40.7 & 6840 & 7.2 & 8382 & 8.8 & 16649 & 17.5 & 8680 & 9.1 & 1710 & 1.8 & 13904 & 14.6 & 181 & 0.2 & 95043 & 100.0 \\
\hline
\end{tabular}




\section{Type of birth}

Table 35 shows type of birth for Local Health Districts, and Table 36 for individual hospitals by maternity service level where at least 200 mothers gave birth in 2009.

\section{Table 35. Type of birth by Local Health District of hospital, NSW 2009}

\begin{tabular}{|c|c|c|c|c|c|c|c|c|c|c|c|c|c|c|}
\hline \multirow[t]{3}{*}{ Local Health District } & \multicolumn{14}{|c|}{ Type of birth } \\
\hline & \multicolumn{2}{|c|}{$\begin{array}{l}\text { Normal } \\
\text { vaginal }\end{array}$} & \multicolumn{2}{|c|}{ Forceps } & \multicolumn{2}{|c|}{$\begin{array}{l}\text { Vacuum } \\
\text { extraction }\end{array}$} & \multicolumn{2}{|c|}{$\begin{array}{l}\text { Vaginal } \\
\text { breech }\end{array}$} & \multicolumn{2}{|c|}{$\begin{array}{l}\text { Elective } \\
\text { caesarean } \\
\text { section }\end{array}$} & \multicolumn{2}{|c|}{$\begin{array}{c}\text { Emergency } \\
\text { caesarean } \\
\text { section\# }\end{array}$} & \multicolumn{2}{|c|}{ TOTAL } \\
\hline & No. & $\%$ & No. & $\%$ & No. & $\%$ & No. & $\%$ & No. & $\%$ & No. & $\%$ & No. & $\%$ \\
\hline Sydney & 3800 & 56.6 & 331 & 4.9 & 542 & 8.1 & 27 & 0.4 & 1075 & 16.0 & 940 & 14.0 & 6715 & 100.0 \\
\hline South Western Sydney & 7049 & 68.1 & 133 & 1.3 & 733 & 7.1 & 45 & 0.4 & 1471 & 14.2 & 926 & 8.9 & 10357 & 100.0 \\
\hline South Eastern Sydney & 4546 & 57.7 & 395 & 5.0 & 701 & 8.9 & 27 & 0.3 & 1175 & 14.9 & 1029 & 13.1 & 7873 & 100.0 \\
\hline Illawarra Shoalhaven & 2183 & 66.3 & 62 & 1.9 & 225 & 6.8 & 5 & 0.2 & 424 & 12.9 & 394 & 12.0 & 3293 & 100.0 \\
\hline Western Sydney & 5612 & 61.5 & 353 & 3.9 & 682 & 7.5 & 59 & 0.6 & 1271 & 13.9 & 1147 & 12.6 & 9124 & 100.0 \\
\hline Nepean Blue Mountains & 2926 & 60.1 & 95 & 2.0 & 351 & 7.2 & 22 & 0.5 & 781 & 16.0 & 696 & 14.3 & 4871 & 100.0 \\
\hline Northern Sydney & 2714 & 56.6 & 272 & 5.7 & 319 & 6.6 & 15 & 0.3 & 754 & 15.7 & 725 & 15.1 & 4799 & 100.0 \\
\hline Central Coast & 1583 & 56.5 & 121 & 4.3 & 165 & 5.9 & 10 & 0.4 & 492 & 17.6 & 431 & 15.4 & 2802 & 100.0 \\
\hline Hunter New England & 5773 & 65.9 & 295 & 3.4 & 444 & 5.1 & 68 & 0.8 & 1094 & 12.5 & 1083 & 12.4 & 8757 & 100.0 \\
\hline Northern NSW & 2525 & 68.0 & 97 & 2.6 & 146 & 3.9 & 19 & 0.5 & 492 & 13.3 & 433 & 11.7 & 3712 & 100.0 \\
\hline Mid North Coast & 1460 & 65.8 & 52 & 2.3 & 147 & 6.6 & 8 & 0.4 & 310 & 14.0 & 242 & 10.9 & 2219 & 100.0 \\
\hline Southern NSW & 952 & 67.5 & 71 & 5.0 & 68 & 4.8 & 0 & 0.0 & 177 & 12.5 & 143 & 10.1 & 1411 & 100.0 \\
\hline Murrumbidgee & 1279 & 64.7 & 37 & 1.9 & 118 & 6.0 & 6 & 0.3 & 306 & 15.5 & 231 & 11.7 & 1977 & 100.0 \\
\hline Western NSW & 2253 & 66.0 & 94 & 2.8 & 140 & 4.1 & 7 & 0.2 & 511 & 15.0 & 411 & 12.0 & 3416 & 100.0 \\
\hline Far West & 140 & 58.8 & 8 & 3.4 & 6 & 2.5 & 0 & 0.0 & 36 & 15.1 & 48 & 20.2 & 238 & 100.0 \\
\hline Private Hospitals & 10400 & 44.6 & 967 & 4.1 & 2445 & 10.5 & 36 & 0.2 & 6280 & 26.9 & 3186 & 13.7 & 23314 & 100.0 \\
\hline TOTAL* & 55359 & 58.2 & 3383 & 3.6 & 7232 & 7.6 & 355 & 0.4 & 16649 & 17.5 & 12065 & 12.7 & 95043 & 100.0 \\
\hline
\end{tabular}

Table 36. Type of birth by hospital and maternity service level, NSW, 2009\#

\begin{tabular}{|c|c|c|c|c|c|c|c|c|c|c|c|c|c|c|}
\hline \multirow{3}{*}{$\begin{array}{l}\text { Maternity service } \\
\text { level-hospital }\end{array}$} & & & \multicolumn{12}{|c|}{ Type of birth } \\
\hline & \multicolumn{2}{|c|}{$\begin{array}{l}\text { Normal } \\
\text { vaginal }\end{array}$} & \multicolumn{2}{|c|}{ Forceps } & \multicolumn{2}{|c|}{$\begin{array}{l}\text { Vacuum } \\
\text { extraction }\end{array}$} & \multicolumn{2}{|c|}{$\begin{array}{l}\text { Vaginal } \\
\text { breech }\end{array}$} & \multicolumn{2}{|c|}{$\begin{array}{l}\text { Elective } \\
\text { caesarean } \\
\text { section }\end{array}$} & \multicolumn{2}{|c|}{$\begin{array}{l}\text { Emergency } \\
\text { caesarean } \\
\text { section\#\# }\end{array}$} & \multicolumn{2}{|c|}{ TOTAL } \\
\hline & No. & $\%$ & No. & $\%$ & No. & $\%$ & No. & $\%$ & No. & $\%$ & No. & $\%$ & No. & $\%$ \\
\hline \multicolumn{15}{|l|}{ Level 6} \\
\hline Royal Prince Alfred & 2720 & 54.2 & 283 & 5.6 & 411 & 8.2 & 27 & 0.5 & 845 & 16.8 & 736 & 14.7 & 5022 & 100.0 \\
\hline Royal North Shore & 1053 & 51.0 & 176 & 8.5 & 74 & 3.6 & 10 & 0.5 & 376 & 18.2 & 377 & 18.2 & 2066 & 100.0 \\
\hline Royal Hospital for Women & 2141 & 52.4 & 308 & 7.5 & 310 & 7.6 & 18 & 0.4 & 712 & 17.4 & 598 & 14.6 & 4087 & 100.0 \\
\hline Liverpool & 2028 & 66.5 & 57 & 1.9 & 167 & 5.5 & 22 & 0.7 & 472 & 15.5 & 302 & 9.9 & 3048 & 100.0 \\
\hline Nepean & 2135 & 59.7 & 60 & 1.7 & 291 & 8.1 & 19 & 0.5 & 573 & 16.0 & 498 & 13.9 & 3576 & 100.0 \\
\hline Westmead & 2626 & 57.2 & 218 & 4.8 & 365 & 8.0 & 44 & 1.0 & 734 & 16.0 & 600 & 13.1 & 4587 & 100.0 \\
\hline John Hunter & 2378 & 64.1 & 194 & 5.2 & 153 & 4.1 & 52 & 1.4 & 413 & 11.1 & 521 & 14.0 & 3711 & 100.0 \\
\hline TOTAL & 15081 & 57.8 & 1296 & 5.0 & 1771 & 6.8 & 192 & 0.7 & 4125 & 15.8 & 3632 & 13.9 & 26097 & 100.0 \\
\hline \multicolumn{15}{|l|}{ Level 5} \\
\hline Gosford & 1350 & 52.6 & 121 & 4.7 & 165 & 6.4 & 10 & 0.4 & 492 & 19.2 & 430 & 16.7 & 2568 & 100.0 \\
\hline St. George & 1566 & 62.5 & 77 & 3.1 & 238 & 9.5 & 6 & 0.2 & 309 & 12.3 & 308 & 12.3 & 2504 & 100.0 \\
\hline Blacktown & 2011 & 62.9 & 112 & 3.5 & 241 & 7.5 & 12 & 0.4 & 399 & 12.5 & 422 & 13.2 & 3197 & 100.0 \\
\hline Lismore Base & 846 & 64.1 & 51 & 3.9 & 40 & 3.0 & 9 & 0.7 & 202 & 15.3 & 171 & 13.0 & 1319 & 100.0 \\
\hline Tweed Heads & 1024 & 68.5 & 30 & 2.0 & 73 & 4.9 & 9 & 0.6 & 172 & 11.5 & 187 & 12.5 & 1495 & 100.0 \\
\hline Port Macquarie Base & 502 & 64.1 & 23 & 2.9 & 60 & 7.7 & 2 & 0.3 & 101 & 12.9 & 95 & 12.1 & 783 & 100.0 \\
\hline Wollongong & 1580 & 67.8 & 25 & 1.1 & 185 & 7.9 & 1 & 0.0 & 256 & 11.0 & 282 & 12.1 & 2329 & 100.0 \\
\hline Maitland & 953 & 62.5 & 35 & 2.3 & 89 & 5.8 & 9 & 0.6 & 214 & 14.0 & 226 & 14.8 & 1526 & 100.0 \\
\hline Wagga Wagga Base & 460 & 60.8 & 15 & 2.0 & 48 & 6.3 & 4 & 0.5 & 121 & 16.0 & 108 & 14.3 & 756 & 100.0 \\
\hline TOTAL & 10292 & 62.5 & 489 & 3.0 & 1139 & 6.9 & 62 & 0.4 & 2266 & 13.8 & 2229 & 13.5 & 16477 & 100.0 \\
\hline \multicolumn{15}{|l|}{ Level 4} \\
\hline Canterbury & 1080 & 63.8 & 48 & 2.8 & 131 & 7.7 & 0 & 0.0 & 230 & 13.6 & 204 & 12.0 & 1693 & 100.0 \\
\hline Hornsby & 703 & 60.7 & 67 & 5.8 & 73 & 6.3 & 2 & 0.2 & 158 & 13.6 & 155 & 13.4 & 1158 & 100.0 \\
\hline Manly & 655 & 57.5 & 25 & 2.2 & 131 & 11.5 & 2 & 0.2 & 166 & 14.6 & 160 & 14.0 & 1139 & 100.0 \\
\hline
\end{tabular}


Table 36. (Continued)

\begin{tabular}{|c|c|c|c|c|c|c|c|c|c|c|c|c|c|c|}
\hline \multirow{3}{*}{$\begin{array}{l}\text { Maternity service } \\
\text { level-hospital }\end{array}$} & & & \multicolumn{12}{|c|}{ Type of birth } \\
\hline & \multicolumn{2}{|c|}{$\begin{array}{l}\text { Normal } \\
\text { vaginal }\end{array}$} & \multicolumn{2}{|c|}{ Forceps } & \multicolumn{2}{|c|}{$\begin{array}{l}\text { Vacuum } \\
\text { extraction }\end{array}$} & \multicolumn{2}{|c|}{$\begin{array}{l}\text { Vaginal } \\
\text { breech }\end{array}$} & \multicolumn{2}{|c|}{$\begin{array}{l}\text { Elective } \\
\text { caesarean } \\
\text { section }\end{array}$} & \multicolumn{2}{|c|}{$\begin{array}{l}\text { Emergency } \\
\text { caesarean } \\
\text { section\#\# }\end{array}$} & \multicolumn{2}{|c|}{ TOTAL } \\
\hline & No. & $\%$ & No. & $\%$ & No. & $\%$ & No. & $\%$ & No. & $\%$ & No. & $\%$ & No. & $\%$ \\
\hline Mona Vale & 182 & 57.8 & 4 & 1.3 & 41 & 13.0 & 1 & 0.3 & 54 & 17.1 & 33 & 10.5 & 315 & 100.0 \\
\hline Sutherland & 839 & 65.4 & 10 & 0.8 & 153 & 11.9 & 3 & 0.2 & 154 & 12.0 & 123 & 9.6 & 1282 & 100.0 \\
\hline Auburn & 975 & 72.8 & 23 & 1.7 & 76 & 5.7 & 3 & 0.2 & 138 & 10.3 & 125 & 9.3 & 1340 & 100.0 \\
\hline Fairfield & 1373 & 73.0 & 15 & 0.8 & 126 & 6.7 & 5 & 0.3 & 224 & 11.9 & 138 & 7.3 & 1881 & 100.0 \\
\hline Campbelltown & 1807 & 67.1 & 14 & 0.5 & 194 & 7.2 & 7 & 0.3 & 420 & 15.6 & 251 & 9.3 & 2693 & 100.0 \\
\hline Bankstown-Lidcombe & 1496 & 68.9 & 14 & 0.6 & 201 & 9.3 & 9 & 0.4 & 274 & 12.6 & 177 & 8.2 & 2171 & 100.0 \\
\hline Hawkesbury & 515 & 59.8 & 30 & 3.5 & 43 & 5.0 & 2 & 0.2 & 132 & 15.3 & 139 & 16.1 & 861 & 100.0 \\
\hline Coffs Harbour & 665 & 61.6 & 28 & 2.6 & 76 & 7.0 & 3 & 0.3 & 180 & 16.7 & 127 & 11.8 & 1079 & 100.0 \\
\hline Grafton Base & 318 & 64.6 & 10 & 2.0 & 25 & 5.1 & 0 & 0.0 & 70 & 14.2 & 69 & 14.0 & 492 & 100.0 \\
\hline Tamworth Base & 512 & 61.3 & 41 & 4.9 & 70 & 8.4 & 0 & 0.0 & 120 & 14.4 & 92 & 11.0 & 835 & 100.0 \\
\hline Manning Base & 493 & 65.5 & 4 & 0.5 & 56 & 7.4 & 1 & 0.1 & 108 & 14.3 & 91 & 12.1 & 753 & 100.0 \\
\hline Dubbo Base & 831 & 69.3 & 40 & 3.3 & 41 & 3.4 & 3 & 0.3 & 163 & 13.6 & 121 & 10.1 & 1199 & 100.0 \\
\hline Orange Base & 512 & 57.7 & 28 & 3.2 & 57 & 6.4 & 1 & 0.1 & 169 & 19.1 & 120 & 13.5 & 887 & 100.0 \\
\hline Goulburn Base & 178 & 58.9 & 32 & 10.6 & 14 & 4.6 & 0 & 0.0 & 44 & 14.6 & 34 & 11.3 & 302 & 100.0 \\
\hline Shoalhaven & 541 & 62.0 & 33 & 3.8 & 27 & 3.1 & 4 & 0.5 & 160 & 18.3 & 108 & 12.4 & 873 & 100.0 \\
\hline Griffith Base & 275 & 67.1 & 14 & 3.4 & 14 & 3.4 & 0 & 0 & 58 & 14.1 & 49 & 12.0 & 410 & 100.0 \\
\hline TOTAL & 13950 & 65.3 & 480 & 2.2 & 1549 & 7.3 & 46 & 0.2 & 3022 & 14.1 & 2316 & 10.8 & 21363 & 100.0 \\
\hline \multicolumn{15}{|l|}{ Level 3} \\
\hline Blue Mountains & 152 & 62.0 & 3 & 1.2 & 15 & 6.1 & 0 & 0.0 & 45 & 18.4 & 30 & 12.2 & 245 & 100.0 \\
\hline Kempsey & 245 & 80.1 & 1 & 0.3 & 9 & 2.9 & 2 & 0.7 & 29 & 9.5 & 20 & 6.5 & 306 & 100.0 \\
\hline Murwillumbah & 149 & 71.6 & 1 & 0.5 & 4 & 1.9 & 0 & 0.0 & 48 & 23.1 & 6 & 2.9 & 208 & 100.0 \\
\hline Armidale & 307 & 70.1 & 3 & 0.7 & 18 & 4.1 & 2 & 0.5 & 60 & 13.7 & 48 & 11.0 & 438 & 100.0 \\
\hline Inverell & 155 & 60.8 & 16 & 6.3 & 3 & 1.2 & 1 & 0.4 & 62 & 24.3 & 18 & 7.1 & 255 & 100.0 \\
\hline Mudgee & 162 & 71.4 & 1 & 0.4 & 14 & 6.2 & 2 & 0.9 & 32 & 14.1 & 16 & 7.0 & 227 & 100.0 \\
\hline Bathurst Base & 355 & 63.4 & 23 & 4.1 & 14 & 2.5 & 1 & 0.2 & 77 & 13.8 & 90 & 16.1 & 560 & 100.0 \\
\hline Bega & 210 & 74.7 & 5 & 1.8 & 13 & 4.6 & 0 & 0.0 & 24 & 8.5 & 29 & 10.3 & 281 & 100.0 \\
\hline Moruya & 202 & 62.2 & 18 & 5.5 & 12 & 3.7 & 0 & 0.0 & 57 & 17.5 & 36 & 11.1 & 325 & 100.0 \\
\hline Queanbeyan & 219 & 70.9 & 14 & 4.5 & 21 & 6.8 & 0 & 0.0 & 28 & 9.1 & 27 & 8.7 & 309 & 100.0 \\
\hline Bowral & 344 & 61.1 & 33 & 5.9 & 45 & 8.0 & 2 & 0.4 & 81 & 14.4 & 58 & 10.3 & 563 & 100.0 \\
\hline Broken Hill Base & 140 & 58.8 & 8 & 3.4 & 6 & 2.5 & 0 & 0.0 & 36 & 15.1 & 48 & 20.2 & 238 & 100.0 \\
\hline Other hospitals & 1797 & 70.7 & 19 & 0.7 & 121 & 4.8 & 6 & 0.2 & 342 & 13.5 & 255 & 10.0 & 2540 & 100.0 \\
\hline TOTAL & 4437 & 68.3 & 145 & 2.2 & 295 & 4.5 & 16 & 0.2 & 921 & 14.2 & 681 & 10.5 & 6495 & 100.0 \\
\hline \multicolumn{15}{|l|}{ Level 2} \\
\hline Wyong & 233 & 99.6 & 0 & 0.0 & 0 & 0.0 & 0 & 0.0 & 0 & 0.0 & 1 & 0.4 & 234 & 100.0 \\
\hline Other hospitals & 759 & 88.9 & 6 & 0.7 & 32 & 3.7 & 2 & 0.2 & 35 & 4.1 & 20 & 2.3 & 854 & 100.0 \\
\hline TOTAL & 992 & 91.2 & 6 & 0.6 & 32 & 2.9 & 2 & 0.2 & 35 & 3.2 & 21 & 1.9 & 1088 & 100.0 \\
\hline \multicolumn{15}{|l|}{ Private } \\
\hline Mater, North Sydney & 899 & 36.0 & 83 & 3.3 & 322 & 12.9 & 1 & 0.0 & 826 & 33.1 & 368 & 14.7 & 2499 & 100.0 \\
\hline North Shore Private & 1128 & 43.0 & 57 & 2.2 & 264 & 10.1 & 5 & 0.2 & 794 & 30.2 & 378 & 14.4 & 2626 & 100.0 \\
\hline Sydney Adventist & 1169 & 52.0 & 99 & 4.4 & 182 & 8.1 & 3 & 0.1 & 496 & 22.1 & 299 & 13.3 & 2248 & 100.0 \\
\hline North Gosford Private & 343 & 41.0 & 11 & 1.3 & 89 & 10.6 & 1 & 0.1 & 287 & 34.3 & 105 & 12.6 & 836 & 100.0 \\
\hline Hurstville Private & 419 & 43.5 & 17 & 1.8 & 117 & 12.1 & 0 & 0 & 244 & 25.3 & 166 & 17.2 & 963 & 100.0 \\
\hline Kareena Private & 252 & 36.9 & 48 & 7.0 & 74 & 10.8 & 1 & 0.1 & 241 & 35.3 & 67 & 9.8 & 683 & 100.0 \\
\hline St. George Private & 682 & 37.5 & 100 & 5.5 & 213 & 11.7 & 1 & 0.1 & 491 & 27.0 & 330 & 18.2 & 1817 & 100.0 \\
\hline Prince of Wales Private & 841 & 37.1 & 101 & 4.5 & 235 & 10.4 & 3 & 0.1 & 708 & 31.2 & 380 & 16.8 & 2268 & 100.0 \\
\hline Norwest Private & 653 & 53.3 & 93 & 7.6 & 133 & 10.9 & 4 & 0.3 & 201 & 16.4 & 141 & 11.5 & 1225 & 100.0 \\
\hline Sydney Southwest Private & 575 & 54.4 & 35 & 3.3 & 116 & 11.0 & 0 & 0 & 238 & 22.5 & 93 & 8.8 & 1057 & 100.0 \\
\hline Nepean Private & 466 & 50.0 & 38 & 4.1 & 66 & 7.1 & 2 & 0.2 & 237 & 25.4 & 123 & 13.2 & 932 & 100.0 \\
\hline Westmead Private & 1162 & 49.1 & 166 & 7.0 & 196 & 8.3 & 3 & 0.1 & 540 & 22.8 & 301 & 12.7 & 2368 & 100.0 \\
\hline Figtree Private & 458 & 45.2 & 15 & 1.5 & 137 & 13.5 & 6 & 0.6 & 258 & 25.5 & 139 & 13.7 & 1013 & 100.0 \\
\hline Newcastle Private & 960 & 51.0 & 48 & 2.5 & 209 & 11.1 & 6 & 0.3 & 451 & 24.0 & 209 & 11.1 & 1883 & 100.0 \\
\hline Calvary, Wagga Wagga & 283 & 45.1 & 38 & 6.1 & 67 & 10.7 & 0 & 0 & 175 & 27.9 & 64 & 10.2 & 627 & 100.0 \\
\hline Other hospitals & 110 & 40.9 & 18 & 6.7 & 25 & 9.3 & 0 & 0 & 93 & 34.6 & 23 & 8.6 & 269 & 100.0 \\
\hline TOTAL & 10400 & 44.6 & 967 & 4.1 & 2445 & 10.5 & 36 & 0.2 & 6280 & 26.9 & 3186 & 13.7 & 23314 & 100.0 \\
\hline TOTAL NSW** & 55359 & 58.2 & 3383 & 3.6 & 7232 & 7.6 & 355 & 0.4 & 16649 & 17.5 & 12065 & 12.7 & 95043 & 100.0 \\
\hline
\end{tabular}




\section{Pain relief}

Table 37 shows type of pain relief provided to women during labour or delivery for Local Health Districts, and Table 38 for individual hospitals by maternity service level where at least 200 mothers gave birth in 2009.
In addition to the types of pain relief shown in the tables, in 2009 a further 23,492 (24.7\%) women were reported to have received local anaesthetic to the perineum, and 747 (0.8\%) received a pudendal block.

Table 37. Pain relief by Local Health District of hospital, NSW 2009

\begin{tabular}{|c|c|c|c|c|c|c|c|c|c|c|c|c|}
\hline \multirow[t]{3}{*}{ Local Health District } & \multicolumn{12}{|c|}{ Pain relief } \\
\hline & \multicolumn{2}{|c|}{$\begin{array}{l}\text { Epidural } \\
\text { - spinal\#\# }\end{array}$} & \multicolumn{2}{|c|}{$\begin{array}{c}\text { General } \\
\text { anaesthetic }\end{array}$} & \multicolumn{2}{|c|}{ IM narcotics } & \multicolumn{2}{|c|}{ Nitrous oxide } & \multicolumn{2}{|c|}{ Nil } & \multicolumn{2}{|c|}{ TOTAL\# } \\
\hline & No. & $\%$ & No. & $\%$ & No. & $\%$ & No. & $\%$ & No. & $\%$ & No. & $\%$ \\
\hline Sydney & 3264 & 48.6 & 288 & 4.3 & 650 & 9.7 & 2739 & 40.8 & 838 & 12.5 & 6715 & 100.0 \\
\hline South Western Sydney & 2915 & 28.1 & 546 & 5.3 & 2867 & 27.7 & 4998 & 48.3 & 1491 & 14.4 & 10357 & 100.0 \\
\hline South Eastern Sydney & 3828 & 48.6 & 190 & 2.4 & 1003 & 12.7 & 3338 & 42.4 & 877 & 11.1 & 7873 & 100.0 \\
\hline Illawarra Shoalhaven & 1202 & 36.5 & 111 & 3.4 & 499 & 15.2 & 1744 & 53.0 & 413 & 12.5 & 3293 & 100.0 \\
\hline Western Sydney & 3746 & 41.1 & 421 & 4.6 & 1355 & 14.9 & 4312 & 47.3 & 675 & 7.4 & 9124 & 100.0 \\
\hline Nepean Blue Mountains & 2082 & 42.7 & 313 & 6.4 & 920 & 18.9 & 2499 & 51.3 & 386 & 7.9 & 4871 & 100.0 \\
\hline Northern Sydney & 2346 & 48.9 & 149 & 3.1 & 714 & 14.9 & 2000 & 41.7 & 427 & 8.9 & 4799 & 100.0 \\
\hline Central Coast & 1234 & 44.0 & 127 & 4.5 & 555 & 19.8 & 1223 & 43.6 & 339 & 12.1 & 2802 & 100.0 \\
\hline Hunter New England & 2752 & 31.4 & 492 & 5.6 & 1837 & 21.0 & 4087 & 46.7 & 1137 & 13.0 & 8757 & 100.0 \\
\hline Northern NSW & 1326 & 35.7 & 102 & 2.7 & 618 & 16.6 & 1780 & 48.0 & 733 & 19.7 & 3712 & 100.0 \\
\hline Mid North Coast & 761 & 34.3 & 59 & 2.7 & 502 & 22.6 & 1106 & 49.8 & 412 & 18.6 & 2219 & 100.0 \\
\hline Southern NSW & 377 & 26.7 & 55 & 3.9 & 252 & 17.9 & 771 & 54.6 & 309 & 21.9 & 1411 & 100.0 \\
\hline Murrumbidgee & 635 & 32.1 & 51 & 2.6 & 602 & 30.5 & 1042 & 52.7 & 317 & 16.0 & 1977 & 100.0 \\
\hline Western NSW & 1124 & 32.9 & 178 & 5.2 & 784 & 23.0 & 1712 & 50.1 & 580 & 17.0 & 3416 & 100.0 \\
\hline Far West & 95 & 39.9 & 11 & 4.6 & 43 & 18.1 & 126 & 52.9 & 31 & 13.0 & 238 & 100.0 \\
\hline Private Hospitals & 15933 & 68.3 & 733 & 3.1 & 2072 & 8.9 & 7546 & 32.4 & 994 & 4.3 & 23314 & 100.0 \\
\hline TOTAL* & 43620 & 45.9 & 3826 & 4.0 & 15273 & 16.1 & 41023 & 43.2 & 10113 & 10.6 & 95043 & 100.0 \\
\hline
\end{tabular}

Table 38. Pain relief by hospital and maternity service level, NSW, 2009\#

\begin{tabular}{|c|c|c|c|c|c|c|c|c|c|c|c|c|}
\hline \multirow{3}{*}{$\begin{array}{l}\text { Maternity service } \\
\text { level-hospital }\end{array}$} & \multicolumn{12}{|c|}{ Pain relief } \\
\hline & \multicolumn{2}{|c|}{$\begin{array}{l}\text { Epidural - } \\
\text { spinal* }\end{array}$} & \multicolumn{2}{|c|}{$\begin{array}{c}\text { General } \\
\text { anaesthetic }\end{array}$} & \multicolumn{2}{|c|}{ IM narcotics } & \multicolumn{2}{|c|}{ Nitrous oxide } & \multicolumn{2}{|c|}{ Nil } & \multicolumn{2}{|c|}{ TOTAL\#\# } \\
\hline & No. & $\%$ & No. & $\%$ & No. & $\%$ & No. & $\%$ & No. & $\%$ & No. & $\%$ \\
\hline \multicolumn{13}{|l|}{ Level 6} \\
\hline Royal Prince Alfred & 2640 & 52.6 & 211 & 4.2 & 466 & 9.3 & 1949 & 38.8 & 636 & 12.7 & 5022 & 100.0 \\
\hline Royal North Shore & 1156 & 56.0 & 76 & 3.7 & 229 & 11.1 & 904 & 43.8 & 99 & 4.8 & 2066 & 100.0 \\
\hline Royal Hospital for Women & 2253 & 55.1 & 80 & 2.0 & 575 & 14.1 & 1492 & 36.5 & 411 & 10.1 & 4087 & 100.0 \\
\hline Liverpool & 1133 & 37.2 & 177 & 5.8 & 1122 & 36.8 & 1175 & 38.5 & 280 & 9.2 & 3048 & 100.0 \\
\hline Nepean & 1586 & 44.4 & 253 & 7.1 & 671 & 18.8 & 1900 & 53.1 & 237 & 6.6 & 3576 & 100.0 \\
\hline Westmead & 2237 & 48.8 & 241 & 5.3 & 621 & 13.5 & 2058 & 44.9 & 151 & 3.3 & 4587 & 100.0 \\
\hline John Hunter & 1284 & 34.6 & 250 & 6.7 & 968 & 26.1 & 1726 & 46.5 & 390 & 10.5 & 3711 & 100.0 \\
\hline TOTAL & 12289 & 47.1 & 1288 & 4.9 & 4652 & 17.8 & 11204 & 42.9 & 2204 & 8.4 & 26097 & 100.0 \\
\hline \multicolumn{13}{|l|}{ Level 5} \\
\hline Gosford & 1234 & 48.1 & 124 & 4.8 & 534 & 20.8 & 1144 & 44.5 & 260 & 10.1 & 2568 & 100.0 \\
\hline St. George & 1067 & 42.6 & 71 & 2.8 & 293 & 11.7 & 1175 & 46.9 & 326 & 13.0 & 2504 & 100.0 \\
\hline Blacktown & 1179 & 36.9 & 130 & 4.1 & 541 & 16.9 & 1553 & 48.6 & 288 & 9.0 & 3197 & 100.0 \\
\hline Lismore Base & 572 & 43.4 & 38 & 2.9 & 197 & 14.9 & 594 & 45.0 & 171 & 13.0 & 1319 & 100.0 \\
\hline Tweed Heads & 534 & 35.7 & 39 & 2.6 & 287 & 19.2 & 772 & 51.6 & 310 & 20.7 & 1495 & 100.0 \\
\hline Port Macquarie Base & 298 & 38.1 & 20 & 2.6 & 211 & 26.9 & 389 & 49.7 & 128 & 16.3 & 783 & 100.0 \\
\hline Wollongong & 859 & 36.9 & 72 & 3.1 & 236 & 10.1 & 1297 & 55.7 & 285 & 12.2 & 2329 & 100.0 \\
\hline Maitland & 520 & 34.1 & 78 & 5.1 & 196 & 12.8 & 739 & 48.4 & 210 & 13.8 & 1526 & 100.0 \\
\hline Wagga Wagga Base & 302 & 39.9 & 16 & 2.1 & 189 & 25.0 & 336 & 44.4 & 137 & 18.1 & 756 & 100.0 \\
\hline TOTAL & 6565 & 39.8 & 588 & 3.6 & 2684 & 16.3 & 7999 & 48.5 & 2115 & 12.8 & 16477 & 100.0 \\
\hline \multicolumn{13}{|l|}{ Level 4} \\
\hline Canterbury & 624 & 36.9 & 77 & 4.5 & 184 & 10.9 & 790 & 46.7 & 202 & 11.9 & 1693 & 100.0 \\
\hline Hornsby & 530 & 45.8 & 36 & 3.1 & 189 & 16.3 & 539 & 46.5 & 83 & 7.2 & 1158 & 100.0 \\
\hline Manly & 523 & 45.9 & 28 & 2.5 & 190 & 16.7 & 401 & 35.2 & 150 & 13.2 & 1139 & 100.0 \\
\hline
\end{tabular}




\begin{tabular}{|c|c|c|c|c|c|c|c|c|c|c|c|c|}
\hline \multirow{3}{*}{$\begin{array}{l}\text { Maternity service } \\
\text { level-hospital }\end{array}$} & \multicolumn{12}{|c|}{ Pain relief } \\
\hline & \multicolumn{2}{|c|}{$\begin{array}{l}\text { Epidural - } \\
\text { spinal* }\end{array}$} & \multicolumn{2}{|c|}{$\begin{array}{c}\text { General } \\
\text { anaesthetic }\end{array}$} & \multicolumn{2}{|c|}{ IM narcotics } & \multicolumn{2}{|c|}{ Nitrous oxide } & \multicolumn{2}{|c|}{ Nil } & \multicolumn{2}{|c|}{ TOTAL\#\# } \\
\hline & No. & $\%$ & No. & $\%$ & No. & $\%$ & No. & $\%$ & No. & $\%$ & No. & $\%$ \\
\hline Mona Vale & 137 & 43.5 & 8 & 2.5 & 102 & 32.4 & 122 & 38.7 & 35 & 11.1 & 315 & 100.0 \\
\hline Sutherland & 508 & 39.6 & 39 & 3.0 & 135 & 10.5 & 671 & 52.3 & 140 & 10.9 & 1282 & 100.0 \\
\hline Auburn & 330 & 24.6 & 50 & 3.7 & 193 & 14.4 & 701 & 52.3 & 236 & 17.6 & 1340 & 100.0 \\
\hline Fairfield & 259 & 13.8 & 143 & 7.6 & 429 & 22.8 & 955 & 50.8 & 333 & 17.7 & 1881 & 100.0 \\
\hline Campbelltown & 747 & 27.7 & 128 & 4.8 & 632 & 23.5 & 1374 & 51.0 & 524 & 19.5 & 2693 & 100.0 \\
\hline Bankstown-Lidcombe & 580 & 26.7 & 81 & 3.7 & 485 & 22.3 & 1230 & 56.7 & 271 & 12.5 & 2171 & 100.0 \\
\hline Hawkesbury & 327 & 38.0 & 45 & 5.2 & 174 & 20.2 & 409 & 47.5 & 86 & 10.0 & 861 & 100.0 \\
\hline Coffs Harbour & 399 & 37.0 & 31 & 2.9 & 180 & 16.7 & 523 & 48.5 & 188 & 17.4 & 1079 & 100.0 \\
\hline Grafton Base & 157 & 31.9 & 23 & 4.7 & 92 & 18.7 & 271 & 55.1 & 94 & 19.1 & 492 & 100.0 \\
\hline Tamworth Base & 371 & 44.4 & 37 & 4.4 & 52 & 6.2 & 422 & 50.5 & 84 & 10.1 & 835 & 100.0 \\
\hline Manning Base & 198 & 26.3 & 53 & 7.0 & 228 & 30.3 & 377 & 50.1 & 97 & 12.9 & 753 & 100.0 \\
\hline Dubbo Base & 399 & 33.3 & 59 & 4.9 & 359 & 29.9 & 610 & 50.9 & 172 & 14.3 & 1199 & 100.0 \\
\hline Orange Base & 360 & 40.6 & 55 & 6.2 & 182 & 20.5 & 442 & 49.8 & 102 & 11.5 & 887 & 100.0 \\
\hline Goulburn Base & 91 & 30.1 & 19 & 6.3 & 59 & 19.5 & 206 & 68.2 & 18 & 6.0 & 302 & 100.0 \\
\hline Shoalhaven & 325 & 37.2 & 38 & 4.4 & 227 & 26.0 & 391 & 44.8 & 120 & 13.7 & 873 & 100.0 \\
\hline Griffith Base & 131 & 32.0 & 13 & 3.2 & 198 & 48.3 & 238 & 58.0 & 48 & 11.7 & 410 & 100.0 \\
\hline TOTAL & 6996 & 32.7 & 963 & 4.5 & 4290 & 20.1 & 10672 & 50.0 & 2983 & 14.0 & 21363 & 100.0 \\
\hline \multicolumn{13}{|l|}{ Level 3} \\
\hline Blue Mountains & 89 & 36.3 & 13 & 5.3 & 34 & 13.9 & 98 & 40.0 & 42 & 17.1 & 245 & 100.0 \\
\hline Kempsey & 64 & 20.9 & 8 & 2.6 & 104 & 34.0 & 174 & 56.9 & 73 & 23.9 & 306 & 100.0 \\
\hline Murwillumbah & 63 & 30.3 & 2 & 1.0 & 35 & 16.8 & 83 & 39.9 & 44 & 21.2 & 208 & 100.0 \\
\hline Armidale & 122 & 27.9 & 15 & 3.4 & 94 & 21.5 & 210 & 47.9 & 47 & 10.7 & 438 & 100.0 \\
\hline Inverell & 81 & 31.8 & 1 & 0.4 & 60 & 23.5 & 96 & 37.6 & 43 & 16.9 & 255 & 100.0 \\
\hline Mudgee & 46 & 20.3 & 5 & 2.2 & 62 & 27.3 & 142 & 62.6 & 33 & 14.5 & 227 & 100.0 \\
\hline Bathurst Base & 193 & 34.5 & 25 & 4.5 & 61 & 10.9 & 265 & 47.3 & 127 & 22.7 & 560 & 100.0 \\
\hline Bega & 62 & 22.1 & 8 & 2.8 & 67 & 23.8 & 176 & 62.6 & 50 & 17.8 & 281 & 100.0 \\
\hline Moruya & 103 & 31.7 & 17 & 5.2 & 68 & 20.9 & 151 & 46.5 & 73 & 22.5 & 325 & 100.0 \\
\hline Queanbeyan & 67 & 21.7 & 9 & 2.9 & 45 & 14.6 & 136 & 44.0 & 113 & 36.6 & 309 & 100.0 \\
\hline Bowral & 196 & 34.8 & 17 & 3.0 & 199 & 35.3 & 264 & 46.9 & 82 & 14.6 & 563 & 100.0 \\
\hline Broken Hill Base & 95 & 39.9 & 11 & 4.6 & 43 & 18.1 & 126 & 52.9 & 31 & 13.0 & 238 & 100.0 \\
\hline Other hospitals & 584 & 23.0 & 114 & 4.5 & 563 & 22.2 & 1275 & 50.2 & 513 & 20.2 & 2540 & 100.0 \\
\hline TOTAL & 1765 & 27.2 & 245 & 3.8 & 1435 & 22.1 & 3196 & 49.2 & 1271 & 19.6 & 6495 & 100.0 \\
\hline \multicolumn{13}{|l|}{ Level 2} \\
\hline Wyong & 0 & 0.0 & 3 & 1.3 & 21 & 9.0 & 79 & 33.8 & 79 & 33.8 & 234 & 100.0 \\
\hline Other hospitals & 72 & 8.4 & 6 & 0.7 & 114 & 13.3 & 319 & 37.4 & 281 & 32.9 & 854 & 100.0 \\
\hline TOTAL & 72 & 6.6 & 9 & 0.8 & 135 & 12.4 & 398 & 36.6 & 360 & 33.1 & 1088 & 100.0 \\
\hline \multicolumn{13}{|l|}{ Private } \\
\hline Mater, North Sydney & 1801 & 72.1 & 36 & 1.4 & 234 & 9.4 & 821 & 32.9 & 97 & 3.9 & 2499 & 100.0 \\
\hline North Shore Private & 1907 & 72.6 & 36 & 1.4 & 113 & 4.3 & 752 & 28.6 & 75 & 2.9 & 2626 & 100.0 \\
\hline Sydney Adventist & 1564 & 69.6 & 30 & 1.3 & 187 & 8.3 & 716 & 31.9 & 62 & 2.8 & 2248 & 100.0 \\
\hline North Gosford Private & 462 & 55.3 & 74 & 8.9 & 68 & 8.1 & 242 & 28.9 & 95 & 11.4 & 836 & 100.0 \\
\hline Hurstville Private & 740 & 76.8 & 17 & 1.8 & 66 & 6.9 & 228 & 23.7 & 41 & 4.3 & 963 & 100.0 \\
\hline Kareena Private & 577 & 84.5 & 8 & 1.2 & 23 & 3.4 & 99 & 14.5 & 29 & 4.2 & 683 & 100.0 \\
\hline St. George Private & 1490 & 82.0 & 40 & 2.2 & 94 & 5.2 & 444 & 24.4 & 63 & 3.5 & 1817 & 100.0 \\
\hline Prince of Wales Private & 1667 & 73.5 & 282 & 12.4 & 51 & 2.2 & 541 & 23.9 & 94 & 4.1 & 2268 & 100.0 \\
\hline Norwest Private & 789 & 64.4 & 47 & 3.8 & 96 & 7.8 & 339 & 27.7 & 71 & 5.8 & 1225 & 100.0 \\
\hline Sydney Southwest Private & 514 & 48.6 & 15 & 1.4 & 333 & 31.5 & 549 & 51.9 & 37 & 3.5 & 1057 & 100.0 \\
\hline Nepean Private & 499 & 53.5 & 22 & 2.4 & 193 & 20.7 & 482 & 51.7 & 57 & 6.1 & 932 & 100.0 \\
\hline Westmead Private & 1528 & 64.5 & 56 & 2.4 & 173 & 7.3 & 925 & 39.1 & 83 & 3.5 & 2368 & 100.0 \\
\hline Figtree Private & 709 & 70.0 & 22 & 2.2 & 27 & 2.7 & 313 & 30.9 & 42 & 4.1 & 1013 & 100.0 \\
\hline Newcastle Private & 1159 & 61.6 & 24 & 1.3 & 274 & 14.6 & 782 & 41.5 & 61 & 3.2 & 1883 & 100.0 \\
\hline Calvary, Wagga Wagga & 364 & 58.1 & 12 & 1.9 & 129 & 20.6 & 215 & 34.3 & 65 & 10.4 & 627 & 100.0 \\
\hline Other hospitals & 163 & 60.6 & 12 & 4.5 & 11 & 4.1 & 98 & 36.4 & 22 & 8.2 & 269 & 100.0 \\
\hline TOTAL & 15933 & 68.3 & 733 & 3.1 & 2072 & 8.9 & 7546 & 32.4 & 994 & 4.3 & 23314 & 100.0 \\
\hline TOTAL NSW* * & 43620 & 45.9 & 3826 & 4.0 & 15269 & 16.1 & 41017 & 43.2 & 10101 & 10.6 & 95043 & 100.0 \\
\hline
\end{tabular}




\section{Perineal status}

Table 39 shows the perineal status in vaginal births for Local Health Districts, and Table 40 for individual hospitals by maternity service level where at least 200 mothers gave birth in 2009.
In 2009, there were 1,560 cases of third degree tear and 116 cases of fourth degree tear reported among women who gave birth vaginally in 2009 , including tears associated with an episiotomy.

\section{Table 39. Perineal status among vaginal births by Local Health District of hospital, NSW 2009}

\begin{tabular}{|c|c|c|c|c|c|c|c|c|c|c|c|c|c|c|c|c|c|c|}
\hline \multirow[t]{3}{*}{ Local Health District } & \multicolumn{18}{|c|}{ Perineal status } \\
\hline & \multicolumn{2}{|c|}{ Intact } & \multicolumn{2}{|c|}{$\begin{array}{l}\text { 1st degree } \\
\text { tear/ graze }\end{array}$} & \multicolumn{2}{|c|}{$\begin{array}{c}\text { 2nd degree } \\
\text { tear }\end{array}$} & \multicolumn{2}{|c|}{$\begin{array}{l}\text { 3rd or 4th } \\
\text { degree tear }\end{array}$} & \multicolumn{2}{|c|}{ Episiotomy } & \multicolumn{2}{|c|}{$\begin{array}{l}\text { Combined } \\
\text { tear and } \\
\text { episiotomy }\end{array}$} & \multicolumn{2}{|c|}{ Other } & \multicolumn{2}{|c|}{ Not stated } & \multicolumn{2}{|c|}{ TOTAL } \\
\hline & No. & $\%$ & No. & $\%$ & No. & $\%$ & No. & $\%$ & No. & $\%$ & No. & $\%$ & No. & $\%$ & No. & $\%$ & No. & $\%$ \\
\hline Sydney & 836 & 17.8 & 1178 & 25.1 & 1750 & 37.2 & 120 & 2.6 & 524 & 11.1 & 111 & 2.4 & 181 & 3.9 & 0 & 0.0 & 4700 & 100.0 \\
\hline South Western Sydney & 2263 & 28.4 & 1875 & 23.6 & 2029 & 25.5 & 121 & 1.5 & 1184 & 14.9 & 149 & 1.9 & 339 & 4.3 & 0 & 0.0 & 7960 & 100.0 \\
\hline South Eastern Sydney & 1157 & 20.4 & 1657 & 29.2 & 1610 & 28.4 & 155 & 2.7 & 759 & 13.4 & 262 & 4.6 & 69 & 1.2 & 0 & 0.0 & 5669 & 100.0 \\
\hline Illawarra Shoalhaven & 743 & 30.0 & 903 & 36.5 & 539 & 21.8 & 30 & 1.2 & 159 & 6.4 & 64 & 2.6 & 37 & 1.5 & 0 & 0.0 & 2475 & 100.0 \\
\hline Western Sydney & 1458 & 21.7 & 2012 & 30.0 & 1702 & 25.4 & 80 & 1.2 & 1023 & 15.3 & 341 & 5.1 & 90 & 1.3 & 0 & 0.0 & 6706 & 100.0 \\
\hline Nepean Blue Mountains & 852 & 25.1 & 1276 & 37.6 & 719 & 21.2 & 49 & 1.4 & 325 & 9.6 & 133 & 3.9 & 40 & 1.2 & 0 & 0.0 & 3394 & 100.0 \\
\hline Northern Sydney & 601 & 18.1 & 1128 & 34.0 & 918 & 27.7 & 73 & 2.2 & 362 & 10.9 & 212 & 6.4 & 26 & 0.8 & 0 & 0.0 & 3320 & 100.0 \\
\hline Central Coast & 397 & 21.1 & 805 & 42.8 & 419 & 22.3 & 31 & 1.6 & 103 & 5.5 & 93 & 4.9 & 31 & 1.6 & 0 & 0.0 & 1879 & 100.0 \\
\hline Hunter New England & 1637 & 24.9 & 2709 & 41.2 & 1334 & 20.3 & 144 & 2.2 & 468 & 7.1 & 204 & 3.1 & 84 & 1.3 & 0 & 0.0 & 6580 & 100.0 \\
\hline Northern NSW & 895 & 32.1 & 779 & 28.0 & 742 & 26.6 & 52 & 1.9 & 220 & 7.9 & 30 & 1.1 & 69 & 2.5 & 0 & 0.0 & 2787 & 100.0 \\
\hline Mid North Coast & 633 & 38.0 & 366 & 22.0 & 434 & 26.0 & 30 & 1.8 & 124 & 7.4 & 40 & 2.4 & 40 & 2.4 & 0 & 0.0 & 1667 & 100.0 \\
\hline Southern NSW & 430 & 39.4 & 247 & 22.6 & 238 & 21.8 & 11 & 1.0 & 125 & 11.5 & 18 & 1.6 & 22 & 2.0 & 0 & 0.0 & 1091 & 100.0 \\
\hline Murrumbidgee & 578 & 40.1 & 426 & 29.6 & 244 & 16.9 & 18 & 1.3 & 131 & 9.1 & 25 & 1.7 & 18 & 1.3 & 0 & 0.0 & 1440 & 100.0 \\
\hline Western NSW & 901 & 36.1 & 701 & 28.1 & 525 & 21.1 & 36 & 1.4 & 193 & 7.7 & 72 & 2.9 & 65 & 2.6 & 1 & 0.0 & 2494 & 100.0 \\
\hline Far West & 75 & 48.7 & 38 & 24.7 & 21 & 13.6 & 1 & 0.6 & 17 & 11.0 & 0 & 0.0 & 2 & 1.3 & 0 & 0.0 & 154 & 100.0 \\
\hline Private Hospitals & 2739 & 19.8 & 2724 & 19.7 & 4285 & 30.9 & 122 & 0.9 & 3417 & 24.7 & 285 & 2.1 & 276 & 2.0 & 0 & 0.0 & 13848 & 100.0 \\
\hline TOTAL* & 16301 & 24.6 & 18857 & 28.4 & 17528 & 26.4 & 1074 & 1.6 & 9134 & 13.8 & 2040 & 3.1 & 1392 & 2.1 & 3 & 0.0 & 66329 & 100.0 \\
\hline
\end{tabular}

Table 40. Perineal status among vaginal births by hospital and maternity service level, NSW, 2009\#

\begin{tabular}{|c|c|c|c|c|c|c|c|c|c|c|c|c|c|c|c|c|c|c|}
\hline \multirow{3}{*}{$\begin{array}{l}\text { Maternity service } \\
\text { level-hospital }\end{array}$} & \multicolumn{18}{|c|}{ Perineal status } \\
\hline & \multicolumn{2}{|c|}{ Intact } & \multicolumn{2}{|c|}{$\begin{array}{l}\text { 1st degree } \\
\text { tear/ graze }\end{array}$} & \multicolumn{2}{|c|}{$\begin{array}{c}\text { 2nd degree } \\
\text { tear }\end{array}$} & \multicolumn{2}{|c|}{$\begin{array}{l}\text { 3rd or } 4 \text { th } \\
\text { degree tear }\end{array}$} & \multicolumn{2}{|c|}{ Episiotomy } & \multicolumn{2}{|c|}{$\begin{array}{l}\text { Combined } \\
\text { tear and } \\
\text { episiotomy }\end{array}$} & \multicolumn{2}{|c|}{ Other } & \multicolumn{2}{|c|}{ Not stated } & \multicolumn{2}{|c|}{ TOTAL } \\
\hline & No. & $\%$ & No. & $\%$ & No. & $\%$ & No. & $\%$ & No. & $\%$ & No. & $\%$ & No. & $\%$ & No. & $\%$ & No. & $\%$ \\
\hline \multicolumn{19}{|l|}{ Level 6} \\
\hline Royal Prince Alfred & 528 & 15.3 & 914 & 26.6 & 1342 & 39.0 & 105 & 3.1 & 356 & 10.3 & 80 & 2.3 & 116 & 3.4 & 0 & 0.0 & 3441 & 100.0 \\
\hline Royal North Shore & 189 & 14.4 & 399 & 30.4 & 363 & 27.6 & 33 & 2.5 & 201 & 15.3 & 117 & 8.9 & 11 & 0.8 & 0 & 0.0 & 1313 & 100.0 \\
\hline $\begin{array}{l}\text { Royal Hospital for } \\
\text { Women }\end{array}$ & 472 & 17.0 & 812 & 29.2 & 775 & 27.9 & 73 & 2.6 & 485 & 17.5 & 119 & 4.3 & 41 & 1.5 & 0 & 0.0 & 2777 & 100.0 \\
\hline Liverpool & 603 & 26.5 & 514 & 22.6 & 540 & 23.7 & 39 & 1.7 & 377 & 16.6 & 56 & 2.5 & 145 & 6.4 & 0 & 0.0 & 2274 & 100.0 \\
\hline Nepean & 595 & 23.8 & 957 & 38.2 & 535 & 21.4 & 40 & 1.6 & 250 & 10.0 & 98 & 3.9 & 30 & 1.2 & 0 & 0.0 & 2505 & 100.0 \\
\hline Westmead & 666 & 20.5 & 806 & 24.8 & 883 & 27.1 & 33 & 1.0 & 609 & 18.7 & 192 & 5.9 & 64 & 2.0 & 0 & 0.0 & 3253 & 100.0 \\
\hline John Hunter & 558 & 20.1 & 1212 & 43.6 & 580 & 20.9 & 86 & 3.1 & 201 & 7.2 & 113 & 4.1 & 27 & 1.0 & 0 & 0.0 & 2777 & 100.0 \\
\hline TOTAL & 3611 & 19.7 & 5614 & 30.6 & 5018 & 27.4 & 409 & 2.2 & 2479 & 13.5 & 775 & 4.2 & 434 & 2.4 & 0 & 0.0 & 18340 & 100.0 \\
\hline \multicolumn{19}{|l|}{ Level 5} \\
\hline Gosford & 343 & 20.8 & 688 & 41.8 & 367 & 22.3 & 30 & 1.8 & 100 & 6.1 & 90 & 5.5 & 28 & 1.7 & 0 & 0.0 & 1646 & 100.0 \\
\hline St. George & 429 & 22.7 & 520 & 27.6 & 561 & 29.7 & 67 & 3.6 & 181 & 9.6 & 114 & 6.0 & 15 & 0.8 & 0 & 0.0 & 1887 & 100.0 \\
\hline Blacktown & 436 & 18.4 & 866 & 36.4 & 605 & 25.5 & 39 & 1.6 & 305 & 12.8 & 112 & 4.7 & 13 & 0.5 & 0 & 0.0 & 2376 & 100.0 \\
\hline Lismore Base & 261 & 27.6 & 293 & 31.0 & 277 & 29.3 & 10 & 1.1 & 82 & 8.7 & 14 & 1.5 & 9 & 1.0 & 0 & 0.0 & 946 & 100.0 \\
\hline Tweed Heads & 342 & 30.1 & 296 & 26.1 & 327 & 28.8 & 35 & 3.1 & 83 & 7.3 & 12 & 1.1 & 41 & 3.6 & 0 & 0.0 & 1136 & 100.0 \\
\hline Port Macquarie Base & 250 & 42.6 & 112 & 19.1 & 159 & 27.1 & 11 & 1.9 & 37 & 6.3 & 16 & 2.7 & 2 & 0.3 & 0 & 0.0 & 587 & 100.0 \\
\hline Wollongong & 530 & 29.6 & 626 & 35.0 & 431 & 24.1 & 22 & 1.2 & 108 & 6.0 & 49 & 2.7 & 25 & 1.4 & 0 & 0.0 & 1791 & 100.0 \\
\hline Maitland & 246 & 22.7 & 453 & 41.7 & 242 & 22.3 & 39 & 3.6 & 55 & 5.1 & 33 & 3.0 & 18 & 1.7 & 0 & 0.0 & 1086 & 100.0 \\
\hline Wagga Wagga Base & 187 & 35.5 & 186 & 35.3 & 89 & 16.9 & 5 & 0.9 & 44 & 8.3 & 11 & 2.1 & 5 & 0.9 & 0 & 0.0 & 527 & 100.0 \\
\hline TOTAL & 3024 & 25.2 & 4040 & 33.7 & 3058 & 25.5 & 258 & 2.2 & 995 & 8.3 & 451 & 3.8 & 156 & 1.3 & 0 & 0.0 & 11982 & 100.0 \\
\hline \multicolumn{19}{|l|}{ Level 4} \\
\hline Canterbury & 308 & 24.5 & 264 & 21.0 & 408 & 32.4 & 15 & 1.2 & 168 & 13.3 & 31 & 2.5 & 65 & 5.2 & 0 & 0.0 & 1259 & 100.0 \\
\hline Hornsby & 154 & 18.2 & 303 & 35.9 & 245 & 29.0 & 20 & 2.4 & 87 & 10.3 & 28 & 3.3 & 8 & 0.9 & 0 & 0.0 & 845 & 100.0 \\
\hline Manly & 163 & 20.0 & 284 & 34.9 & 224 & 27.6 & 16 & 2.0 & 62 & 7.6 & 58 & 7.1 & 6 & 0.7 & 0 & 0.0 & 813 & 100.0 \\
\hline
\end{tabular}


Table 40. (Continued)

\begin{tabular}{|c|c|c|c|c|c|c|c|c|c|c|c|c|c|c|c|c|c|c|}
\hline \multirow{3}{*}{$\begin{array}{l}\text { Maternity service } \\
\text { level-hospital }\end{array}$} & \multicolumn{18}{|c|}{ Perineal status } \\
\hline & \multicolumn{2}{|l|}{ Intact } & \multicolumn{2}{|c|}{$\begin{array}{l}\text { 1st degree } \\
\text { tear/ graze }\end{array}$} & \multicolumn{2}{|c|}{$\begin{array}{c}\text { 2nd degree } \\
\text { tear }\end{array}$} & \multicolumn{2}{|c|}{$\begin{array}{l}\text { 3rd or } 4 \text { th } \\
\text { degree tear }\end{array}$} & \multicolumn{2}{|c|}{ Episiotomy } & \multicolumn{2}{|c|}{$\begin{array}{l}\text { Combined } \\
\text { tear and } \\
\text { episiotomy }\end{array}$} & \multicolumn{2}{|c|}{ Other } & \multicolumn{2}{|c|}{ Not stated } & \multicolumn{2}{|c|}{ TOTAL } \\
\hline & No. & $\%$ & No. & $\%$ & No. & $\%$ & No. & $\%$ & No. & $\%$ & No. & $\%$ & No. & $\%$ & No. & $\%$ & No. & $\%$ \\
\hline Mona Vale & 56 & 24.6 & 86 & 37.7 & 61 & 26.8 & 3 & 1.3 & 12 & 5.3 & 9 & 3.9 & 1 & 0.4 & 0 & 0.0 & 228 & 100.0 \\
\hline Sutherland & 256 & 25.5 & 325 & 32.3 & 274 & 27.3 & 15 & 1.5 & 93 & 9.3 & 29 & 2.9 & 13 & 1.3 & 0 & 0.0 & 1005 & 100.0 \\
\hline Auburn & 356 & 33.1 & 340 & 31.6 & 214 & 19.9 & 8 & 0.7 & 109 & 10.1 & 37 & 3.4 & 13 & 1.2 & 0 & 0.0 & 1077 & 100.0 \\
\hline Fairfield & 452 & 29.8 & 364 & 24.0 & 407 & 26.8 & 23 & 1.5 & 230 & 15.1 & 17 & 1.1 & 26 & 1.7 & 0 & 0.0 & 1519 & 100.0 \\
\hline Campbelltown & 601 & 29.7 & 521 & 25.8 & 543 & 26.9 & 29 & 1.4 & 234 & 11.6 & 18 & 0.9 & 76 & 3.8 & 0 & 0.0 & 2022 & 100.0 \\
\hline Bankstown-Lidcombe & 463 & 26.9 & 359 & 20.9 & 437 & 25.4 & 26 & 1.5 & 297 & 17.3 & 54 & 3.1 & 84 & 4.9 & 0 & 0.0 & 1720 & 100.0 \\
\hline Hawkesbury & 154 & 26.1 & 232 & 39.3 & 111 & 18.8 & 7 & 1.2 & 52 & 8.8 & 30 & 5.1 & 4 & 0.7 & 0 & 0.0 & 590 & 100.0 \\
\hline Coffs Harbour & 237 & 30.7 & 182 & 23.6 & 201 & 26.0 & 19 & 2.5 & 78 & 10.1 & 21 & 2.7 & 34 & 4.4 & 0 & 0.0 & 772 & 100.0 \\
\hline Grafton Base & 147 & 41.6 & 84 & 23.8 & 69 & 19.5 & 6 & 1.7 & 32 & 9.1 & 0 & 0 & 15 & 4.2 & 0 & 0.0 & 353 & 100.0 \\
\hline Tamworth Base & 135 & 21.7 & 274 & 44.0 & 117 & 18.8 & 9 & 1.4 & 66 & 10.6 & 19 & 3.0 & 3 & 0.5 & 0 & 0.0 & 623 & 100.0 \\
\hline Manning Base & 185 & 33.4 & 203 & 36.6 & 116 & 20.9 & 1 & 0.2 & 28 & 5.1 & 10 & 1.8 & 11 & 2.0 & 0 & 0.0 & 554 & 100.0 \\
\hline Dubbo Base & 340 & 37.2 & 232 & 25.4 & 171 & 18.7 & 14 & 1.5 & 95 & 10.4 & 27 & 3.0 & 35 & 3.8 & 1 & 0.1 & 915 & 100.0 \\
\hline Orange Base & 171 & 28.6 & 198 & 33.1 & 142 & 23.7 & 9 & 1.5 & 41 & 6.9 & 30 & 5.0 & 7 & 1.2 & 0 & 0.0 & 598 & 100.0 \\
\hline Goulburn Base & 70 & 31.3 & 25 & 11.2 & 36 & 16.1 & 4 & 1.8 & 80 & 35.7 & 5 & 2.2 & 4 & 1.8 & 0 & 0.0 & 224 & 100.0 \\
\hline Shoalhaven & 180 & 29.8 & 254 & 42.0 & 92 & 15.2 & 6 & 1.0 & 49 & 8.1 & 13 & 2.1 & 11 & 1.8 & 0 & 0.0 & 605 & 100.0 \\
\hline Griffith Base & 116 & 38.3 & 108 & 35.6 & 41 & 13.5 & 10 & 3.3 & 22 & 7.3 & 3 & 1.0 & 3 & 1.0 & 0 & 0.0 & 303 & 100.0 \\
\hline TOTAL & 4544 & 28.4 & 4638 & 28.9 & 3909 & 24.4 & 240 & 1.5 & 1835 & 11.5 & 439 & 2.7 & 419 & 2.6 & 1 & 0.0 & 16025 & 100.0 \\
\hline Level 3 & & & & & & & & & & & & & & & & & & \\
\hline Blue Mountains & 48 & 28.2 & 61 & 35.9 & 46 & 27.1 & 2 & 1.2 & 8 & 4.7 & 3 & 1.8 & 2 & 1.2 & 0 & 0.0 & 170 & 100.0 \\
\hline Kempsey & 121 & 47.1 & 57 & 22.2 & 65 & 25.3 & 0 & 0.0 & 9 & 3.5 & 3 & 1.2 & 2 & 0.8 & 0 & 0.0 & 257 & 100.0 \\
\hline Murwillumbah & 71 & 46.1 & 38 & 24.7 & 30 & 19.5 & 1 & 0.6 & 9 & 5.8 & 1 & 0.6 & 4 & 2.6 & 0 & 0.0 & 154 & 100.0 \\
\hline Armidale & 114 & 34.5 & 111 & 33.6 & 62 & 18.8 & 1 & 0.3 & 28 & 8.5 & 11 & 3.3 & 3 & 0.9 & 0 & 0.0 & 330 & 100.0 \\
\hline Inverell & 64 & 36.6 & 47 & 26.9 & 16 & 9.1 & 0 & 0.0 & 36 & 20.6 & 5 & 2.9 & 7 & 4.0 & 0 & 0.0 & 175 & 100.0 \\
\hline Mudgee & 78 & 43.6 & 46 & 25.7 & 43 & 24.0 & 0 & 0.0 & 6 & 3.4 & 5 & 2.8 & 1 & 0.6 & 0 & 0.0 & 179 & 100.0 \\
\hline Bathurst Base & 139 & 35.4 & 124 & 31.6 & 65 & 16.5 & 7 & 1.8 & 41 & 10.4 & 3 & 0.8 & 14 & 3.6 & 0 & 0.0 & 393 & 100.0 \\
\hline Bega & 102 & 44.7 & 50 & 21.9 & 51 & 22.4 & 1 & 0.4 & 14 & 6.1 & 4 & 1.8 & 6 & 2.6 & 0 & 0.0 & 228 & 100.0 \\
\hline Moruya & 98 & 42.2 & 54 & 23.3 & 54 & 23.3 & 2 & 0.9 & 17 & 7.3 & 1 & 0.4 & 6 & 2.6 & 0 & 0.0 & 232 & 100.0 \\
\hline Queanbeyan & 87 & 34.3 & 82 & 32.3 & 59 & 23.2 & 2 & 0.8 & 10 & 3.9 & 8 & 3.1 & 6 & 2.4 & 0 & 0.0 & 254 & 100.0 \\
\hline Bowral & 143 & 33.7 & 117 & 27.6 & 102 & 24.1 & 4 & 0.9 & 46 & 10.8 & 4 & 0.9 & 8 & 1.9 & 0 & 0.0 & 424 & 100.0 \\
\hline Broken Hill Base & 75 & 48.7 & 38 & 24.7 & 21 & 13.6 & 1 & 0.6 & 17 & 11.0 & 0 & 0 & 2 & 1.3 & 0 & 0.0 & 154 & 100.0 \\
\hline Other hospitals & 778 & 40.0 & 534 & 27.5 & 407 & 20.9 & 13 & 0.7 & 148 & 7.6 & 29 & 1.5 & 34 & 1.7 & 0 & 0.0 & 1943 & 100.0 \\
\hline TOTAL & 1918 & 39.2 & 1359 & 27.8 & 1021 & 20.9 & 34 & 0.7 & 389 & 8.0 & 77 & 1.6 & 95 & 1.9 & 0 & 0.0 & 4893 & 100.0 \\
\hline Level 2 & & & & & & & & & & & & & & & & & & \\
\hline Wyong & 54 & 23.2 & 117 & 50.2 & 52 & 22.3 & 1 & 0.4 & 3 & 1.3 & 3 & 1.3 & 3 & 1.3 & 0 & 0.0 & 233 & 100.0 \\
\hline Other hospitals & 276 & 34.5 & 321 & 40.2 & 164 & 20.5 & 9 & 1.1 & 14 & 1.8 & 9 & 1.1 & 6 & 0.8 & 0 & 0.0 & 799 & 100.0 \\
\hline TOTAL & 330 & 32.0 & 438 & 42.4 & 216 & 20.9 & 10 & 1.0 & 17 & 1.6 & 12 & 1.2 & 9 & 0.9 & 0 & 0.0 & 1032 & 100.0 \\
\hline Private & & & & & & & & & & & & & & & & & & \\
\hline Mater, North Sydney & 184 & 14.1 & 236 & 18.1 & 354 & 27.1 & 11 & 0.8 & 397 & 30.4 & 69 & 5.3 & 54 & 4.1 & 0 & 0.0 & 1305 & 100.0 \\
\hline North Shore Private & 251 & 17.3 & 306 & 21.0 & 496 & 34.1 & 13 & 0.9 & 373 & 25.7 & 5 & 0.3 & 10 & 0.7 & 0 & 0.0 & 1454 & 100.0 \\
\hline Sydney Adventist & 259 & 17.8 & 394 & 27.1 & 443 & 30.5 & 13 & 0.9 & 316 & 21.7 & 23 & 1.6 & 5 & 0.3 & 0 & 0.0 & 1453 & 100.0 \\
\hline North Gosford Private & 143 & 32.2 & 99 & 22.3 & 115 & 25.9 & 3 & 0.7 & 65 & 14.6 & 8 & 1.8 & 11 & 2.5 & 0 & 0.0 & 444 & 100.0 \\
\hline Hurstville Private & 84 & 15.2 & 114 & 20.6 & 142 & 25.7 & 6 & 1.1 & 164 & 29.7 & 26 & 4.7 & 17 & 3.1 & 0 & 0.0 & 553 & 100.0 \\
\hline Kareena Private & 76 & 20.3 & 83 & 22.1 & 129 & 34.4 & 5 & 1.3 & 76 & 20.3 & 3 & 0.8 & 3 & 0.8 & 0 & 0.0 & 375 & 100.0 \\
\hline St. George Private & 235 & 23.6 & 213 & 21.4 & 318 & 31.9 & 17 & 1.7 & 194 & 19.5 & 4 & 0.4 & 15 & 1.5 & 0 & 0.0 & 996 & 100.0 \\
\hline $\begin{array}{l}\text { Prince of Wales } \\
\text { Private }\end{array}$ & 245 & 20.8 & 242 & 20.5 & 310 & 26.3 & 2 & 0.2 & 277 & 23.5 & 55 & 4.7 & 49 & 4.2 & 0 & 0.0 & 1180 & 100.0 \\
\hline Norwest Private & 211 & 23.9 & 154 & 17.4 & 328 & 37.1 & 6 & 0.7 & 147 & 16.6 & 16 & 1.8 & 21 & 2.4 & 0 & 0.0 & 883 & 100.0 \\
\hline $\begin{array}{l}\text { Sydney Southwest } \\
\text { Private }\end{array}$ & 141 & 19.4 & 128 & 17.6 & 167 & 23.0 & 6 & 0.8 & 235 & 32.4 & 21 & 2.9 & 28 & 3.9 & 0 & 0.0 & 726 & 100.0 \\
\hline Nepean Private & 119 & 20.8 & 43 & 7.5 & 185 & 32.3 & 4 & 0.7 & 183 & 32.0 & 7 & 1.2 & 31 & 5.4 & 0 & 0.0 & 572 & 100.0 \\
\hline Westmead Private & 270 & 17.7 & 295 & 19.3 & 452 & 29.6 & 3 & 0.2 & 493 & 32.3 & 4 & 0.3 & 10 & 0.7 & 0 & 0.0 & 1527 & 100.0 \\
\hline Figtree Private & 129 & 20.9 & 96 & 15.6 & 191 & 31.0 & 3 & 0.5 & 195 & 31.7 & 1 & 0.2 & 1 & 0.2 & 0 & 0.0 & 616 & 100.0 \\
\hline Newcastle Private & 303 & 24.8 & 240 & 19.6 & 455 & 37.2 & 18 & 1.5 & 196 & 16.0 & 2 & 0.2 & 9 & 0.7 & 0 & 0.0 & 1223 & 100.0 \\
\hline $\begin{array}{l}\text { Calvary, Wagga } \\
\text { Wagga }\end{array}$ & 63 & 16.2 & 55 & 14.2 & 158 & 40.7 & 12 & 3.1 & 60 & 15.5 & 33 & 8.5 & 7 & 1.8 & 0 & 0.0 & 388 & 100.0 \\
\hline Other hospitals & 26 & 17.0 & 26 & 17.0 & 42 & 27.5 & 0 & 0 & 46 & 30.1 & 8 & 5.2 & 5 & 3.3 & 0 & 0.0 & 153 & 100.0 \\
\hline TOTAL & 2739 & 19.8 & 2724 & 19.7 & 4285 & 30.9 & 122 & 0.9 & 3417 & 24.7 & 285 & 2.1 & 276 & 2.0 & 0 & 0.0 & 13848 & 100.0 \\
\hline TOTAL NSW* & 16301 & 24.6 & 18857 & 28.4 & 17528 & 26.4 & 1074 & 1.6 & 9134 & 13.8 & 2040 & 3.1 & 1392 & 2.1 & 3 & 0.0 & 66329 & 100.0 \\
\hline
\end{tabular}




\section{Birth weight}

Table 41 shows the birth weight of babies for Local Health service level where at least 200 mothers gave birth in 2009 . Districts, and Table 42 for individual hospitals by maternity

Table 41. Births by birth weight and Local Health District of hospital, NSW 2009

\begin{tabular}{|c|c|c|c|c|c|c|c|c|c|c|c|c|}
\hline \multirow[t]{3}{*}{ Local Health District } & \multicolumn{12}{|c|}{ Birth weight (grams) } \\
\hline & \multicolumn{2}{|c|}{ Less than 1000} & \multicolumn{2}{|c|}{ 1000-1499 } & \multicolumn{2}{|c|}{$1500-2499$} & \multicolumn{2}{|c|}{$2500+$} & \multicolumn{2}{|c|}{ Not stated } & \multicolumn{2}{|c|}{ TOTAL } \\
\hline & No. & $\%$ & No. & $\%$ & No. & $\%$ & No. & $\%$ & No. & $\%$ & No. & $\%$ \\
\hline Sydney & 71 & 1.0 & 57 & 0.8 & 362 & 5.3 & 6314 & 92.7 & 9 & 0.1 & 6813 & 100.0 \\
\hline South Western Sydney & 93 & 0.9 & 76 & 0.7 & 551 & 5.2 & 9781 & 93.1 & 8 & 0.1 & 10509 & 100.0 \\
\hline South Eastern Sydney & 83 & 1.0 & 71 & 0.9 & 462 & 5.7 & 7416 & 92.2 & 9 & 0.1 & 8041 & 100.0 \\
\hline Illawarra Shoalhaven & 18 & 0.5 & 8 & 0.2 & 173 & 5.2 & 3142 & 94.0 & 3 & 0.1 & 3344 & 100.0 \\
\hline Western Sydney & 99 & 1.1 & 79 & 0.8 & 540 & 5.8 & 8575 & 92.2 & 11 & 0.1 & 9304 & 100.0 \\
\hline Nepean Blue Mountains & 50 & 1.0 & 43 & 0.9 & 308 & 6.2 & 4555 & 91.9 & 1 & 0.0 & 4957 & 100.0 \\
\hline Northern Sydney & 53 & 1.1 & 43 & 0.9 & 209 & 4.3 & 4548 & 93.7 & 1 & 0.0 & 4854 & 100.0 \\
\hline Central Coast & 13 & 0.5 & 5 & 0.2 & 127 & 4.5 & 2678 & 94.7 & 5 & 0.2 & 2828 & 100.0 \\
\hline Hunter New England & 89 & 1.0 & 74 & 0.8 & 550 & 6.2 & 8164 & 91.9 & 9 & 0.1 & 8886 & 100.0 \\
\hline Northern NSW & 19 & 0.5 & 8 & 0.2 & 173 & 4.6 & 3556 & 94.6 & 1 & 0.0 & 3757 & 100.0 \\
\hline Mid North Coast & 14 & 0.6 & 3 & 0.1 & 91 & 4.1 & 2131 & 95.1 & 2 & 0.1 & 2241 & 100.0 \\
\hline Southern NSW & 1 & 0.1 & 3 & 0.2 & 39 & 2.8 & 1368 & 97.0 & 0 & 0.0 & 1411 & 100.0 \\
\hline Murrumbidgee & 8 & 0.4 & 6 & 0.3 & 84 & 4.2 & 1896 & 95.1 & 0 & 0.0 & 1994 & 100.0 \\
\hline Western NSW & 6 & 0.2 & 11 & 0.3 & 161 & 4.7 & 3280 & 94.7 & 4 & 0.1 & 3462 & 100.0 \\
\hline Far West & 1 & 0.4 & 0 & 0.0 & 15 & 6.2 & 226 & 93.4 & 0 & 0.0 & 242 & 100.0 \\
\hline Private Hospitals & 43 & 0.2 & 14 & 0.1 & 777 & 3.3 & 22787 & 96.4 & 9 & 0.0 & 23630 & 100.0 \\
\hline Other & 0 & 0.0 & 0 & 0.0 & 0 & 0.0 & 164 & 98.8 & 2 & 1.2 & 166 & 100.0 \\
\hline TOTAL* & 661 & 0.7 & 501 & 0.5 & 4622 & 4.8 & 90581 & 93.9 & 74 & 0.1 & 96439 & 100.0 \\
\hline
\end{tabular}

Table 42. Births by birth weight, hospital and maternity service level, NSW 2009\#

\begin{tabular}{|c|c|c|c|c|c|c|c|c|c|c|c|c|}
\hline \multirow[t]{3}{*}{ Maternity service level-hospital } & \multicolumn{12}{|c|}{ Birth weight (grams) } \\
\hline & \multicolumn{2}{|c|}{ Less than 1000} & \multicolumn{2}{|c|}{ 1000-1499 } & \multicolumn{2}{|c|}{$1500-2499$} & \multicolumn{2}{|c|}{$2500+$} & \multicolumn{2}{|c|}{ Not stated } & \multicolumn{2}{|c|}{ TOTAL } \\
\hline & No. & $\%$ & No. & $\%$ & No. & $\%$ & No. & $\%$ & No. & $\%$ & No. & $\%$ \\
\hline \multicolumn{13}{|l|}{ Level 6} \\
\hline Royal Prince Alfred & 65 & 1.3 & 56 & 1.1 & 302 & 5.9 & 4681 & 91.6 & 7 & 0.1 & 5111 & 100.0 \\
\hline Royal North Shore & 48 & 2.3 & 42 & 2.0 & 130 & 6.2 & 1885 & 89.5 & 1 & 0.0 & 2106 & 100.0 \\
\hline Royal Hospital for Women & 64 & 1.5 & 61 & 1.5 & 281 & 6.7 & 3792 & 90.2 & 5 & 0.1 & 4203 & 100.0 \\
\hline Liverpool & 63 & 2.0 & 69 & 2.2 & 270 & 8.6 & 2739 & 87.2 & 0 & 0.0 & 3141 & 100.0 \\
\hline Nepean & 47 & 1.3 & 43 & 1.2 & 259 & 7.1 & 3303 & 90.4 & 1 & 0.0 & 3653 & 100.0 \\
\hline Westmead & 80 & 1.7 & 75 & 1.6 & 349 & 7.4 & 4210 & 89.1 & 10 & 0.2 & 4724 & 100.0 \\
\hline John Hunter & 73 & 1.9 & 64 & 1.7 & 317 & 8.4 & 3332 & 87.9 & 6 & 0.2 & 3792 & 100.0 \\
\hline TOTAL & 440 & 1.6 & 410 & 1.5 & 1908 & 7.1 & 23942 & 89.6 & 30 & 0.1 & 26730 & 100.0 \\
\hline \multicolumn{13}{|l|}{ Level 5} \\
\hline Gosford & 13 & 0.5 & 5 & 0.2 & 126 & 4.9 & 2445 & 94.3 & 5 & 0.2 & 2594 & 100.0 \\
\hline St. George & 15 & 0.6 & 9 & 0.4 & 146 & 5.7 & 2382 & 93.2 & 4 & 0.2 & 2556 & 100.0 \\
\hline Blacktown & 15 & 0.5 & 4 & 0.1 & 153 & 4.7 & 3064 & 94.7 & 0 & 0.0 & 3236 & 100.0 \\
\hline Lismore Base & 6 & 0.4 & 5 & 0.4 & 65 & 4.8 & 1265 & 94.3 & 0 & 0.0 & 1341 & 100.0 \\
\hline Tweed Heads & 11 & 0.7 & 3 & 0.2 & 72 & 4.8 & 1428 & 94.3 & 1 & 0.1 & 1515 & 100.0 \\
\hline Port Macquarie Base & 8 & 1.0 & 1 & 0.1 & 39 & 4.9 & 748 & 93.9 & 1 & 0.1 & 797 & 100.0 \\
\hline Wollongong & 8 & 0.3 & 5 & 0.2 & 137 & 5.8 & 2213 & 93.5 & 3 & 0.1 & 2366 & 100.0 \\
\hline Maitland & 4 & 0.3 & 2 & 0.1 & 73 & 4.7 & 1466 & 94.8 & 1 & 0.1 & 1546 & 100.0 \\
\hline Wagga Wagga Base & 7 & 0.9 & 5 & 0.7 & 39 & 5.1 & 714 & 93.3 & 0 & 0.0 & 765 & 100.0 \\
\hline TOTAL & 87 & 0.5 & 39 & 0.2 & 850 & 5.1 & 15725 & 94.1 & 15 & 0.1 & 16716 & 100.0 \\
\hline \multicolumn{13}{|l|}{ Level 4} \\
\hline Canterbury & 6 & 0.4 & 1 & 0.1 & 60 & 3.5 & 1633 & 95.9 & 2 & 0.1 & 1702 & 100.0 \\
\hline Hornsby & 3 & 0.3 & 0 & 0.0 & 39 & 3.3 & 1125 & 96.4 & 0 & 0.0 & 1167 & 100.0 \\
\hline Manly & 1 & 0.1 & 1 & 0.1 & 28 & 2.4 & 1114 & 97.4 & 0 & 0.0 & 1144 & 100.0 \\
\hline
\end{tabular}


Table 42. (Continued)

\begin{tabular}{|c|c|c|c|c|c|c|c|c|c|c|c|c|}
\hline \multirow[t]{3}{*}{ Maternity service level-hospital } & \multicolumn{12}{|c|}{ Birth weight (grams) } \\
\hline & \multicolumn{2}{|c|}{ Less than 1000} & \multicolumn{2}{|c|}{$1000-1499$} & \multicolumn{2}{|c|}{$1500-2499$} & \multicolumn{2}{|c|}{$2500+$} & \multicolumn{2}{|c|}{ Not stated } & \multicolumn{2}{|c|}{ TOTAL } \\
\hline & No. & $\%$ & No. & $\%$ & No. & $\%$ & No. & $\%$ & No. & $\%$ & No. & $\%$ \\
\hline Mona Vale & 1 & 0.3 & 0 & 0.0 & 11 & 3.5 & 304 & 96.2 & 0 & 0.0 & 316 & 100.0 \\
\hline Sutherland & 4 & 0.3 & 1 & 0.1 & 35 & 2.7 & 1242 & 96.9 & 0 & 0.0 & 1282 & 100.0 \\
\hline Auburn & 4 & 0.3 & 0 & 0.0 & 38 & 2.8 & 1301 & 96.8 & 1 & 0.1 & 1344 & 100.0 \\
\hline Fairfield & 6 & 0.3 & 3 & 0.2 & 75 & 4.0 & 1809 & 95.5 & 1 & 0.1 & 1894 & 100.0 \\
\hline Campbelltown & 10 & 0.4 & 2 & 0.1 & 118 & 4.4 & 2577 & 95.1 & 3 & 0.1 & 2710 & 100.0 \\
\hline Bankstown-Lidcombe & 14 & 0.6 & 2 & 0.1 & 76 & 3.5 & 2098 & 95.6 & 4 & 0.2 & 2194 & 100.0 \\
\hline Hawkesbury & 3 & 0.3 & 0 & 0.0 & 28 & 3.2 & 837 & 96.4 & 0 & 0.0 & 868 & 100.0 \\
\hline Coffs Harbour & 5 & 0.5 & 2 & 0.2 & 37 & 3.4 & 1041 & 95.9 & 1 & 0.1 & 1086 & 100.0 \\
\hline Grafton Base & 2 & 0.4 & 0 & 0.0 & 28 & 5.7 & 464 & 93.9 & 0 & 0.0 & 494 & 100.0 \\
\hline Tamworth Base & 3 & 0.4 & 5 & 0.6 & 54 & 6.4 & 782 & 92.7 & 0 & 0.0 & 844 & 100.0 \\
\hline Manning Base & 3 & 0.4 & 0 & 0.0 & 47 & 6.2 & 709 & 93.3 & 1 & 0.1 & 760 & 100.0 \\
\hline Dubbo Base & 3 & 0.2 & 5 & 0.4 & 64 & 5.2 & 1149 & 94.1 & 0 & 0.0 & 1221 & 100.0 \\
\hline Orange Base & 1 & 0.1 & 3 & 0.3 & 58 & 6.4 & 838 & 92.8 & 3 & 0.3 & 903 & 100.0 \\
\hline Goulburn Base & 0 & 0.0 & 1 & 0.3 & 10 & 3.3 & 291 & 96.4 & 0 & 0.0 & 302 & 100.0 \\
\hline Shoalhaven & 10 & 1.1 & 3 & 0.3 & 34 & 3.8 & 840 & 94.7 & 0 & 0.0 & 887 & 100.0 \\
\hline Griffith Base & 1 & 0.2 & 0 & 0.0 & 19 & 4.6 & 395 & 95.2 & 0 & 0.0 & 415 & 100.0 \\
\hline TOTAL & 80 & 0.4 & 29 & 0.1 & 859 & 4.0 & 20549 & 95.4 & 16 & 0.1 & 21533 & 100.0 \\
\hline \multicolumn{13}{|l|}{ Level 3} \\
\hline Blue Mountains & 0 & 0.0 & 0 & 0.0 & 6 & 2.4 & 240 & 97.6 & 0 & 0.0 & 246 & 100.0 \\
\hline Kempsey & 1 & 0.3 & 0 & 0.0 & 11 & 3.6 & 295 & 96.1 & 0 & 0.0 & 307 & 100.0 \\
\hline Murwillumbah & 0 & 0.0 & 0 & 0.0 & 8 & 3.8 & 201 & 96.2 & 0 & 0.0 & 209 & 100.0 \\
\hline Armidale & 2 & 0.4 & 1 & 0.2 & 27 & 6.0 & 417 & 93.3 & 0 & 0.0 & 447 & 100.0 \\
\hline Inverell & 0 & 0.0 & 2 & 0.8 & 9 & 3.5 & 245 & 95.3 & 1 & 0.4 & 257 & 100.0 \\
\hline Mudgee & 0 & 0.0 & 0 & 0.0 & 4 & 1.7 & 225 & 98.3 & 0 & 0.0 & 229 & 100.0 \\
\hline Bathurst Base & 2 & 0.4 & 2 & 0.4 & 24 & 4.2 & 538 & 95.1 & 0 & 0.0 & 566 & 100.0 \\
\hline Bega & 1 & 0.4 & 1 & 0.4 & 9 & 3.2 & 270 & 96.1 & 0 & 0.0 & 281 & 100.0 \\
\hline Moruya & 0 & 0.0 & 1 & 0.3 & 10 & 3.1 & 314 & 96.6 & 0 & 0.0 & 325 & 100.0 \\
\hline Queanbeyan & 0 & 0.0 & 0 & 0.0 & 3 & 1.0 & 306 & 99.0 & 0 & 0.0 & 309 & 100.0 \\
\hline Bowral & 0 & 0.0 & 0 & 0.0 & 12 & 2.1 & 557 & 97.9 & 0 & 0.0 & 569 & 100.0 \\
\hline Broken Hill Base & 1 & 0.4 & 0 & 0.0 & 15 & 6.2 & 226 & 93.4 & 0 & 0.0 & 242 & 100.0 \\
\hline Other hospitals & 2 & 0.1 & 2 & 0.1 & 72 & 2.8 & 2469 & 97.0 & 0 & 0.0 & 2545 & 100.0 \\
\hline TOTAL & 9 & 0.1 & 9 & 0.1 & 210 & 3.2 & 6303 & 96.5 & 1 & 0.0 & 6532 & 100.0 \\
\hline \multicolumn{13}{|l|}{ Level 2} \\
\hline Wyong & 0 & 0.0 & 0 & 0.0 & 1 & 0.4 & 233 & 99.6 & 0 & 0.0 & 234 & 100.0 \\
\hline Other hospitals & 2 & 0.2 & 0 & 0.0 & 15 & 1.8 & 837 & 98.0 & 0 & 0.0 & 854 & 100.0 \\
\hline TOTAL & 2 & 0.2 & 0 & 0.0 & 16 & 1.5 & 1070 & 98.3 & 0 & 0.0 & 1088 & 100.0 \\
\hline \multicolumn{13}{|l|}{ Private } \\
\hline Mater, North Sydney & 1 & 0.0 & 0 & 0.0 & 84 & 3.3 & 2449 & 96.6 & 2 & 0.1 & 2536 & 100.0 \\
\hline North Shore Private & 5 & 0.2 & 7 & 0.3 & 118 & 4.4 & 2541 & 95.1 & 0 & 0.0 & 2671 & 100.0 \\
\hline Sydney Adventist & 6 & 0.3 & 2 & 0.1 & 78 & 3.4 & 2198 & 96.2 & 2 & 0.1 & 2286 & 100.0 \\
\hline North Gosford Private & 2 & 0.2 & 0 & 0.0 & 27 & 3.2 & 819 & 96.6 & 0 & 0.0 & 848 & 100.0 \\
\hline Hurstville Private & 1 & 0.1 & 1 & 0.1 & 45 & 4.6 & 928 & 95.2 & 0 & 0.0 & 975 & 100.0 \\
\hline Kareena Private & 2 & 0.3 & 0 & 0.0 & 23 & 3.3 & 664 & 96.4 & 0 & 0.0 & 689 & 100.0 \\
\hline St. George Private & 1 & 0.1 & 0 & 0.0 & 71 & 3.8 & 1772 & 96.0 & 1 & 0.1 & 1845 & 100.0 \\
\hline Prince of Wales Private & 1 & 0.0 & 0 & 0.0 & 59 & 2.6 & 2231 & 97.4 & 0 & 0.0 & 2291 & 100.0 \\
\hline Norwest Private & 6 & 0.5 & 1 & 0.1 & 43 & 3.5 & 1189 & 95.8 & 2 & 0.2 & 1241 & 100.0 \\
\hline Sydney Southwest Private & 0 & 0.0 & 0 & 0.0 & 23 & 2.2 & 1046 & 97.8 & 0 & 0.0 & 1069 & 100.0 \\
\hline Nepean Private & 0 & 0.0 & 1 & 0.1 & 19 & 2.0 & 920 & 97.9 & 0 & 0.0 & 940 & 100.0 \\
\hline Westmead Private & 7 & 0.3 & 2 & 0.1 & 89 & 3.7 & 2295 & 95.9 & 0 & 0.0 & 2393 & 100.0 \\
\hline Figtree Private & 5 & 0.5 & 0 & 0.0 & 27 & 2.6 & 1000 & 96.8 & 1 & 0.1 & 1033 & 100.0 \\
\hline Newcastle Private & 4 & 0.2 & 0 & 0.0 & 53 & 2.8 & 1845 & 97.0 & 1 & 0.1 & 1903 & 100.0 \\
\hline Calvary, Wagga Wagga & 2 & 0.3 & 0 & 0.0 & 15 & 2.3 & 622 & 97.3 & 0 & 0.0 & 639 & 100.0 \\
\hline Other hospitals & 0 & 0.0 & 0 & 0.0 & 3 & 1.1 & 268 & 98.9 & 0 & 0.0 & 271 & 100.0 \\
\hline TOTAL & 43 & 0.2 & 14 & 0.1 & 777 & 3.3 & 22787 & 96.4 & 9 & 0.0 & 23630 & 100.0 \\
\hline TOTAL NSW * & 661 & 0.7 & 501 & 0.5 & 4622 & 4.8 & 90581 & 93.9 & 74 & 0.1 & 96439 & 100.0 \\
\hline
\end{tabular}

Source: NSW Perinatal Data Collection (HOIST). Centre for Epidemiology and Research, NSW Ministry of Health.

\# Hospitals with more than 200 births are identified individually. Maternity service levels are described on page 13 of the Methods chapter.

* Total NSW includes births at home assisted by independent midwives and births at level 1 hospitals. 


\section{Gestational age}

Table 43 shows the gestational age of babies for Local Health service level where at least 200 mothers gave birth in 2009.

Districts, and Table 44 for individual hospitals by maternity

Table 43. Births by gestational age and Local Health District of hospital, NSW 2009

\begin{tabular}{|c|c|c|c|c|c|c|c|c|c|c|c|c|}
\hline \multirow[t]{3}{*}{ Local Health District } & \multicolumn{12}{|c|}{ Gestational age (weeks) } \\
\hline & \multicolumn{2}{|c|}{$<32$} & \multicolumn{2}{|c|}{$32-33$} & \multicolumn{2}{|c|}{$34-36$} & \multicolumn{2}{|c|}{$37+$} & \multicolumn{2}{|c|}{ Not stated } & \multicolumn{2}{|c|}{ TOTAL } \\
\hline & No. & $\%$ & No. & $\%$ & No. & $\%$ & No. & $\%$ & No. & $\%$ & No. & $\%$ \\
\hline Sydney & 150 & 2.2 & 90 & 1.3 & 320 & 4.7 & 6253 & 91.8 & 0 & 0.0 & 6813 & 100.0 \\
\hline South Western Sydney & 190 & 1.8 & 94 & 0.9 & 516 & 4.9 & 9708 & 92.4 & 1 & 0.0 & 10509 & 100.0 \\
\hline South Eastern Sydney & 190 & 2.4 & 100 & 1.2 & 450 & 5.6 & 7299 & 90.8 & 2 & 0.0 & 8041 & 100.0 \\
\hline Illawarra Shoalhaven & 32 & 1.0 & 39 & 1.2 & 215 & 6.4 & 3058 & 91.4 & 0 & 0.0 & 3344 & 100.0 \\
\hline Western Sydney & 197 & 2.1 & 102 & 1.1 & 485 & 5.2 & 8520 & 91.6 & 0 & 0.0 & 9304 & 100.0 \\
\hline Nepean Blue Mountains & 122 & 2.5 & 73 & 1.5 & 284 & 5.7 & 4477 & 90.3 & 1 & 0.0 & 4957 & 100.0 \\
\hline Northern Sydney & 103 & 2.1 & 43 & 0.9 & 201 & 4.1 & 4506 & 92.8 & 1 & 0.0 & 4854 & 100.0 \\
\hline Central Coast & 24 & 0.8 & 16 & 0.6 & 150 & 5.3 & 2638 & 93.3 & 0 & 0.0 & 2828 & 100.0 \\
\hline Hunter New England & 205 & 2.3 & 113 & 1.3 & 575 & 6.5 & 7993 & 90.0 & 0 & 0.0 & 8886 & 100.0 \\
\hline Northern NSW & 29 & 0.8 & 23 & 0.6 & 171 & 4.6 & 3534 & 94.1 & 0 & 0.0 & 3757 & 100.0 \\
\hline Mid North Coast & 21 & 0.9 & 5 & 0.2 & 104 & 4.6 & 2111 & 94.2 & 0 & 0.0 & 2241 & 100.0 \\
\hline Southern NSW & 4 & 0.3 & 4 & 0.3 & 43 & 3.0 & 1360 & 96.4 & 0 & 0.0 & 1411 & 100.0 \\
\hline Murrumbidgee & 17 & 0.9 & 7 & 0.4 & 78 & 3.9 & 1892 & 94.9 & 0 & 0.0 & 1994 & 100.0 \\
\hline Western NSW & 15 & 0.4 & 15 & 0.4 & 172 & 5.0 & 3260 & 94.2 & 0 & 0.0 & 3462 & 100.0 \\
\hline Far West & 0 & 0.0 & 0 & 0.0 & 21 & 8.7 & 221 & 91.3 & 0 & 0.0 & 242 & 100.0 \\
\hline Private Hospitals & 66 & 0.3 & 102 & 0.4 & 1085 & 4.6 & 22375 & 94.7 & 2 & 0.0 & 23630 & 100.0 \\
\hline TOTAL* & 1365 & 1.4 & 826 & 0.9 & 4871 & 5.1 & 89369 & 92.7 & 8 & 0.0 & 96439 & 100.0 \\
\hline
\end{tabular}

Table 44. Births by gestational age, hospital and maternity service level, NSW 2009\#

\begin{tabular}{|c|c|c|c|c|c|c|c|c|c|c|c|c|}
\hline \multirow[t]{3}{*}{ Maternity service level-hospital } & \multicolumn{12}{|c|}{ Gestational age (weeks) } \\
\hline & \multicolumn{2}{|c|}{$<32$} & \multicolumn{2}{|c|}{$32-33$} & \multicolumn{2}{|c|}{$34-36$} & \multicolumn{2}{|c|}{$37+$} & \multicolumn{2}{|c|}{ Not stated } & \multicolumn{2}{|c|}{ TOTAL } \\
\hline & No. & $\%$ & No. & $\%$ & No. & $\%$ & No. & $\%$ & No. & $\%$ & No. & $\%$ \\
\hline \multicolumn{13}{|l|}{ Level 6} \\
\hline Royal Prince Alfred & 140 & 2.7 & 89 & 1.7 & 261 & 5.1 & 4621 & 90.4 & 0 & 0.0 & 5111 & 100.0 \\
\hline Royal North Shore & 97 & 4.6 & 39 & 1.9 & 105 & 5.0 & 1864 & 88.5 & 1 & 0.0 & 2106 & 100.0 \\
\hline Royal Hospital for Women & 151 & 3.6 & 71 & 1.7 & 279 & 6.6 & 3701 & 88.1 & 1 & 0.0 & 4203 & 100.0 \\
\hline Liverpool & 143 & 4.6 & 76 & 2.4 & 216 & 6.9 & 2706 & 86.2 & 0 & 0.0 & 3141 & 100.0 \\
\hline Nepean & 119 & 3.3 & 67 & 1.8 & 222 & 6.1 & 3245 & 88.8 & 0 & 0.0 & 3653 & 100.0 \\
\hline Westmead & 170 & 3.6 & 86 & 1.8 & 283 & 6.0 & 4185 & 88.6 & 0 & 0.0 & 4724 & 100.0 \\
\hline John Hunter & 167 & 4.4 & 89 & 2.3 & 295 & 7.8 & 3241 & 85.5 & 0 & 0.0 & 3792 & 100.0 \\
\hline TOTAL & 987 & 3.7 & 517 & 1.9 & 1661 & 6.2 & 23563 & 88.2 & 2 & 0.0 & 26730 & 100.0 \\
\hline \multicolumn{13}{|l|}{ Level 5} \\
\hline Gosford & 24 & 0.9 & 16 & 0.6 & 150 & 5.8 & 2404 & 92.7 & 0 & 0.0 & 2594 & 100.0 \\
\hline St. George & 32 & 1.3 & 25 & 1.0 & 124 & 4.9 & 2374 & 92.9 & 1 & 0.0 & 2556 & 100.0 \\
\hline Blacktown & 21 & 0.6 & 15 & 0.5 & 159 & 4.9 & 3041 & 94.0 & 0 & 0.0 & 3236 & 100.0 \\
\hline Lismore Base & 13 & 1.0 & 6 & 0.4 & 80 & 6.0 & 1242 & 92.6 & 0 & 0.0 & 1341 & 100.0 \\
\hline Tweed Heads & 14 & 0.9 & 9 & 0.6 & 67 & 4.4 & 1425 & 94.1 & 0 & 0.0 & 1515 & 100.0 \\
\hline Port Macquarie Base & 10 & 1.3 & 2 & 0.3 & 41 & 5.1 & 744 & 93.4 & 0 & 0.0 & 797 & 100.0 \\
\hline Wollongong & 19 & 0.8 & 31 & 1.3 & 171 & 7.2 & 2145 & 90.7 & 0 & 0.0 & 2366 & 100.0 \\
\hline Maitland & 6 & 0.4 & 5 & 0.3 & 106 & 6.9 & 1429 & 92.4 & 0 & 0.0 & 1546 & 100.0 \\
\hline Wagga Wagga Base & 12 & 1.6 & 6 & 0.8 & 47 & 6.1 & 700 & 91.5 & 0 & 0.0 & 765 & 100.0 \\
\hline TOTAL & 151 & 0.9 & 115 & 0.7 & 945 & 5.7 & 15504 & 92.7 & 1 & 0.0 & 16716 & 100.0 \\
\hline \multicolumn{13}{|l|}{ Level 4} \\
\hline Canterbury & 10 & 0.6 & 1 & 0.1 & 59 & 3.5 & 1632 & 95.9 & 0 & 0.0 & 1702 & 100.0 \\
\hline Hornsby & 3 & 0.3 & 3 & 0.3 & 47 & 4.0 & 1114 & 95.5 & 0 & 0.0 & 1167 & 100.0 \\
\hline Manly & 2 & 0.2 & 0 & 0.0 & 34 & 3.0 & 1108 & 96.9 & 0 & 0.0 & 1144 & 100.0 \\
\hline
\end{tabular}


Table 44. (Continued)

\begin{tabular}{|c|c|c|c|c|c|c|c|c|c|c|c|c|}
\hline \multirow[t]{3}{*}{ Maternity service level-hospital } & \multicolumn{12}{|c|}{ Gestational age (weeks) } \\
\hline & \multicolumn{2}{|c|}{$<32$} & \multicolumn{2}{|c|}{$32-33$} & \multicolumn{2}{|c|}{$34-36$} & \multicolumn{2}{|c|}{$37+$} & \multicolumn{2}{|c|}{ Not stated } & \multicolumn{2}{|c|}{ TOTAL } \\
\hline & No. & $\%$ & No. & $\%$ & No. & $\%$ & No. & $\%$ & No. & $\%$ & No. & $\%$ \\
\hline Mona Vale & 1 & 0.3 & 1 & 0.3 & 14 & 4.4 & 300 & 94.9 & 0 & 0.0 & 316 & 100.0 \\
\hline Sutherland & 7 & 0.5 & 4 & 0.3 & 47 & 3.7 & 1224 & 95.5 & 0 & 0.0 & 1282 & 100.0 \\
\hline Auburn & 6 & 0.4 & 1 & 0.1 & 43 & 3.2 & 1294 & 96.3 & 0 & 0.0 & 1344 & 100.0 \\
\hline Fairfield & 10 & 0.5 & 3 & 0.2 & 75 & 4.0 & 1806 & 95.4 & 0 & 0.0 & 1894 & 100.0 \\
\hline Campbelltown & 18 & 0.7 & 10 & 0.4 & 126 & 4.6 & 2556 & 94.3 & 0 & 0.0 & 2710 & 100.0 \\
\hline Bankstown-Lidcombe & 19 & 0.9 & 5 & 0.2 & 88 & 4.0 & 2081 & 94.8 & 1 & 0.0 & 2194 & 100.0 \\
\hline Hawkesbury & 3 & 0.3 & 1 & 0.1 & 39 & 4.5 & 825 & 95.0 & 0 & 0.0 & 868 & 100.0 \\
\hline Coffs Harbour & 9 & 0.8 & 1 & 0.1 & 49 & 4.5 & 1027 & 94.6 & 0 & 0.0 & 1086 & 100.0 \\
\hline Grafton Base & 2 & 0.4 & 8 & 1.6 & 17 & 3.4 & 467 & 94.5 & 0 & 0.0 & 494 & 100.0 \\
\hline Tamworth Base & 12 & 1.4 & 6 & 0.7 & 55 & 6.5 & 771 & 91.4 & 0 & 0.0 & 844 & 100.0 \\
\hline Manning Base & 8 & 1.1 & 8 & 1.1 & 45 & 5.9 & 699 & 92.0 & 0 & 0.0 & 760 & 100.0 \\
\hline Dubbo Base & 6 & 0.5 & 5 & 0.4 & 72 & 5.9 & 1138 & 93.2 & 0 & 0.0 & 1221 & 100.0 \\
\hline Orange Base & 5 & 0.6 & 6 & 0.7 & 67 & 7.4 & 825 & 91.4 & 0 & 0.0 & 903 & 100.0 \\
\hline Goulburn Base & 1 & 0.3 & 1 & 0.3 & 11 & 3.6 & 289 & 95.7 & 0 & 0.0 & 302 & 100.0 \\
\hline Shoalhaven & 13 & 1.5 & 7 & 0.8 & 42 & 4.7 & 825 & 93.0 & 0 & 0.0 & 887 & 100.0 \\
\hline Griffith Base & 1 & 0.2 & 1 & 0.2 & 13 & 3.1 & 400 & 96.4 & 0 & 0.0 & 415 & 100.0 \\
\hline TOTAL & 136 & 0.6 & 72 & 0.3 & 943 & 4.4 & 20381 & 94.7 & 1 & 0.0 & 21533 & 100.0 \\
\hline \multicolumn{13}{|l|}{ Level 3} \\
\hline Blue Mountains & 0 & 0.0 & 1 & 0.4 & 11 & 4.5 & 233 & 94.7 & 1 & 0.4 & 246 & 100.0 \\
\hline Kempsey & 2 & 0.7 & 1 & 0.3 & 13 & 4.2 & 291 & 94.8 & 0 & 0.0 & 307 & 100.0 \\
\hline Murwillumbah & 0 & 0.0 & 0 & 0.0 & 7 & 3.3 & 202 & 96.7 & 0 & 0.0 & 209 & 100.0 \\
\hline Armidale & 4 & 0.9 & 2 & 0.4 & 30 & 6.7 & 411 & 91.9 & 0 & 0.0 & 447 & 100.0 \\
\hline Inverell & 3 & 1.2 & 2 & 0.8 & 13 & 5.1 & 239 & 93.0 & 0 & 0.0 & 257 & 100.0 \\
\hline Mudgee & 0 & 0.0 & 0 & 0.0 & 4 & 1.7 & 225 & 98.3 & 0 & 0.0 & 229 & 100.0 \\
\hline Bathurst Base & 4 & 0.7 & 2 & 0.4 & 19 & 3.4 & 541 & 95.6 & 0 & 0.0 & 566 & 100.0 \\
\hline Bega & 1 & 0.4 & 1 & 0.4 & 9 & 3.2 & 270 & 96.1 & 0 & 0.0 & 281 & 100.0 \\
\hline Moruya & 2 & 0.6 & 1 & 0.3 & 9 & 2.8 & 313 & 96.3 & 0 & 0.0 & 325 & 100.0 \\
\hline Queanbeyan & 0 & 0.0 & 0 & 0.0 & 10 & 3.2 & 299 & 96.8 & 0 & 0.0 & 309 & 100.0 \\
\hline Bowral & 0 & 0.0 & 0 & 0.0 & 11 & 1.9 & 558 & 98.1 & 0 & 0.0 & 569 & 100.0 \\
\hline Broken Hill Base & 0 & 0.0 & 0 & 0.0 & 21 & 8.7 & 221 & 91.3 & 0 & 0.0 & 242 & 100.0 \\
\hline Other hospitals & 6 & 0.2 & 6 & 0.2 & 62 & 2.4 & 2471 & 97.1 & 0 & 0.0 & 2545 & 100.0 \\
\hline TOTAL & 22 & 0.3 & 16 & 0.2 & 219 & 3.4 & 6274 & 96.1 & 1 & 0.0 & 6532 & 100.0 \\
\hline \multicolumn{13}{|l|}{ Level 2} \\
\hline Wyong & 0 & 0.0 & 0 & 0.0 & 0 & 0.0 & 234 & 100.0 & 0 & 0.0 & 234 & 100.0 \\
\hline Other hospitals & 3 & 0.4 & 3 & 0.4 & 13 & 1.5 & 835 & 97.8 & 0 & 0.0 & 854 & 100.0 \\
\hline TOTAL & 3 & 0.3 & 3 & 0.3 & 13 & 1.2 & 1069 & 98.3 & 0 & 0.0 & 1088 & 100.0 \\
\hline \multicolumn{13}{|l|}{ Private } \\
\hline Mater, North Sydney & 3 & 0.1 & 23 & 0.9 & 106 & 4.2 & 2403 & 94.8 & 1 & 0.0 & 2536 & 100.0 \\
\hline North Shore Private & 9 & 0.3 & 29 & 1.1 & 140 & 5.2 & 2493 & 93.3 & 0 & 0.0 & 2671 & 100.0 \\
\hline Sydney Adventist & 12 & 0.5 & 13 & 0.6 & 80 & 3.5 & 2181 & 95.4 & 0 & 0.0 & 2286 & 100.0 \\
\hline North Gosford Private & 2 & 0.2 & 1 & 0.1 & 41 & 4.8 & 804 & 94.8 & 0 & 0.0 & 848 & 100.0 \\
\hline Hurstville Private & 1 & 0.1 & 4 & 0.4 & 59 & 6.1 & 911 & 93.4 & 0 & 0.0 & 975 & 100.0 \\
\hline Kareena Private & 2 & 0.3 & 2 & 0.3 & 49 & 7.1 & 636 & 92.3 & 0 & 0.0 & 689 & 100.0 \\
\hline St. George Private & 2 & 0.1 & 8 & 0.4 & 84 & 4.6 & 1751 & 94.9 & 0 & 0.0 & 1845 & 100.0 \\
\hline Prince of Wales Private & 1 & 0.0 & 4 & 0.2 & 94 & 4.1 & 2191 & 95.6 & 1 & 0.0 & 2291 & 100.0 \\
\hline Norwest Private & 8 & 0.6 & 8 & 0.6 & 50 & 4.0 & 1175 & 94.7 & 0 & 0.0 & 1241 & 100.0 \\
\hline Sydney Southwest Private & 1 & 0.1 & 0 & 0.0 & 52 & 4.9 & 1016 & 95.0 & 0 & 0.0 & 1069 & 100.0 \\
\hline Nepean Private & 1 & 0.1 & 0 & 0.0 & 40 & 4.3 & 899 & 95.6 & 0 & 0.0 & 940 & 100.0 \\
\hline Westmead Private & 9 & 0.4 & 4 & 0.2 & 120 & 5.0 & 2260 & 94.4 & 0 & 0.0 & 2393 & 100.0 \\
\hline Figtree Private & 6 & 0.6 & 0 & 0.0 & 42 & 4.1 & 985 & 95.4 & 0 & 0.0 & 1033 & 100.0 \\
\hline Newcastle Private & 6 & 0.3 & 1 & 0.1 & 82 & 4.3 & 1814 & 95.3 & 0 & 0.0 & 1903 & 100.0 \\
\hline Calvary, Wagga Wagga & 3 & 0.5 & 5 & 0.8 & 39 & 6.1 & 592 & 92.6 & 0 & 0.0 & 639 & 100.0 \\
\hline Other hospitals & 0 & 0.0 & 0 & 0.0 & 7 & 2.6 & 264 & 97.4 & 0 & 0.0 & 271 & 100.0 \\
\hline TOTAL & 66 & 0.3 & 102 & 0.4 & 1085 & 4.6 & 22375 & 94.7 & 2 & 0.0 & 23630 & 100.0 \\
\hline TOTAL NSW* & 1365 & 1.4 & 826 & 0.9 & 4871 & 5.1 & 89369 & 92.7 & 8 & 0.0 & 96439 & 100.0 \\
\hline
\end{tabular}




\section{Neonatal resuscitation}

Table 45 shows the type of neonatal resuscitation for Local Health Districts, and Table 46 for individual hospitals by maternity service level where at least 200 mothers gave birth in 2009 .

Table 45. Births by type of resuscitation and Local Health District of hospital, NSW 2009

\begin{tabular}{|c|c|c|c|c|c|c|c|c|c|c|c|c|c|c|c|c|}
\hline \multirow[t]{3}{*}{ Local Health District } & \multicolumn{16}{|c|}{ Resuscitation } \\
\hline & \multicolumn{2}{|c|}{ None } & \multicolumn{2}{|c|}{ Suction } & \multicolumn{2}{|c|}{$\begin{array}{l}\text { Oxygen } \\
\text { therapy }\end{array}$} & \multicolumn{2}{|c|}{$\begin{array}{l}\text { IPPR by bag } \\
\text { and mask }\end{array}$} & \multicolumn{2}{|c|}{$\begin{array}{l}\text { Intubation } \\
\text { and IPPR }\end{array}$} & \multicolumn{2}{|c|}{$\begin{array}{c}\text { External cardiac } \\
\text { massage and } \\
\text { ventilation }\end{array}$} & \multicolumn{2}{|c|}{ Not stated } & \multicolumn{2}{|c|}{ Total } \\
\hline & No. & $\%$ & No. & $\%$ & No. & $\%$ & No. & $\%$ & No. & $\%$ & No. & $\%$ & No. & $\%$ & No. & $\%$ \\
\hline Sydney & 5348 & 78.5 & 310 & 4.6 & 453 & 6.6 & 581 & 8.5 & 78 & 1.1 & 43 & 0.6 & 0 & 0.0 & 6813 & 100.0 \\
\hline South Western Sydney & 8868 & 84.4 & 498 & 4.7 & 585 & 5.6 & 465 & 4.4 & 44 & 0.4 & 48 & 0.5 & 1 & 0.0 & 10509 & 100.0 \\
\hline South Eastern Sydney & 6146 & 76.4 & 574 & 7.1 & 718 & 8.9 & 492 & 6.1 & 90 & 1.1 & 21 & 0.3 & 0 & 0.0 & 8041 & 100.0 \\
\hline Illawarra Shoalhaven & 2445 & 73.1 & 432 & 12.9 & 298 & 8.9 & 145 & 4.3 & 8 & 0.2 & 16 & 0.5 & 0 & 0.0 & 3344 & 100.0 \\
\hline Western Sydney & 6808 & 73.2 & 948 & 10.2 & 877 & 9.4 & 472 & 5.1 & 161 & 1.7 & 38 & 0.4 & 0 & 0.0 & 9304 & 100.0 \\
\hline Nepean Blue Mountains & 3415 & 68.9 & 690 & 13.9 & 527 & 10.6 & 242 & 4.9 & 60 & 1.2 & 23 & 0.5 & 0 & 0.0 & 4957 & 100.0 \\
\hline Northern Sydney & 3534 & 72.8 & 434 & 8.9 & 501 & 10.3 & 345 & 7.1 & 23 & 0.5 & 17 & 0.4 & 0 & 0.0 & 4854 & 100.0 \\
\hline Central Coast & 2080 & 73.6 & 167 & 5.9 & 313 & 11.1 & 239 & 8.5 & 12 & 0.4 & 17 & 0.6 & 0 & 0.0 & 2828 & 100.0 \\
\hline Hunter New England & 6457 & 72.7 & 289 & 3.3 & 1030 & 11.6 & 1010 & 11.4 & 57 & 0.6 & 43 & 0.5 & 0 & 0.0 & 8886 & 100.0 \\
\hline Northern NSW & 2755 & 73.3 & 211 & 5.6 & 502 & 13.4 & 270 & 7.2 & 12 & 0.3 & 7 & 0.2 & 0 & 0.0 & 3757 & 100.0 \\
\hline Mid North Coast & 1761 & 78.6 & 91 & 4.1 & 231 & 10.3 & 145 & 6.5 & 5 & 0.2 & 8 & 0.4 & 0 & 0.0 & 2241 & 100.0 \\
\hline Southern NSW & 1206 & 85.5 & 43 & 3.0 & 87 & 6.2 & 69 & 4.9 & 2 & 0.1 & 4 & 0.3 & 0 & 0.0 & 1411 & 100.0 \\
\hline Murrumbidgee & 1479 & 74.2 & 139 & 7.0 & 229 & 11.5 & 132 & 6.6 & 6 & 0.3 & 9 & 0.5 & 0 & 0.0 & 1994 & 100.0 \\
\hline Western NSW & 2693 & 77.8 & 120 & 3.5 & 415 & 12.0 & 211 & 6.1 & 12 & 0.3 & 11 & 0.3 & 0 & 0.0 & 3462 & 100.0 \\
\hline Far West & 177 & 73.1 & 25 & 10.3 & 27 & 11.2 & 12 & 5.0 & 0 & 0.0 & 1 & 0.4 & 0 & 0.0 & 242 & 100.0 \\
\hline Private Hospitals & 15931 & 67.4 & 3774 & 16.0 & 2898 & 12.3 & 978 & 4.1 & 27 & 0.1 & 16 & 0.1 & 6 & 0.0 & 23630 & 100.0 \\
\hline TOTAL* & 71257 & 73.9 & 8746 & 9.1 & 9698 & 10.1 & 5812 & 6.0 & 597 & 0.6 & 322 & 0.3 & 7 & 0.0 & 96439 & 100.0 \\
\hline
\end{tabular}

Table 46. Births by type of resuscitation, hospital and maternity service level, NSW 2009\#

\begin{tabular}{|c|c|c|c|c|c|c|c|c|c|c|c|c|c|c|c|c|}
\hline \multirow{3}{*}{$\begin{array}{l}\text { Maternity service level - } \\
\text { hospital }\end{array}$} & \multicolumn{16}{|c|}{ Resuscitation } \\
\hline & \multicolumn{2}{|c|}{ None } & \multicolumn{2}{|c|}{ Suction } & \multicolumn{2}{|c|}{$\begin{array}{l}\text { Oxygen } \\
\text { therapy }\end{array}$} & \multicolumn{2}{|c|}{$\begin{array}{l}\text { IPPR by bag } \\
\text { and mask }\end{array}$} & \multicolumn{2}{|c|}{$\begin{array}{l}\text { Intubation } \\
\text { and IPPR }\end{array}$} & \multicolumn{2}{|c|}{$\begin{array}{c}\text { External cardiac } \\
\text { massage and } \\
\text { ventilation }\end{array}$} & \multicolumn{2}{|c|}{ Not stated } & \multicolumn{2}{|c|}{ TOTAL } \\
\hline & No. & $\%$ & No. & $\%$ & No. & $\%$ & No. & $\%$ & No. & $\%$ & No. & $\%$ & No. & $\%$ & No. & $\%$ \\
\hline \multicolumn{17}{|l|}{ Level 6} \\
\hline Royal Prince Alfred & 3882 & 76.0 & 262 & 5.1 & 311 & 6.1 & 546 & 10.7 & 76 & 1.5 & 34 & 0.7 & 0 & 0.0 & 5111 & 100.0 \\
\hline Royal North Shore & 1460 & 69.3 & 268 & 12.7 & 167 & 7.9 & 179 & 8.5 & 19 & 0.9 & 13 & 0.6 & 0 & 0.0 & 2106 & 100.0 \\
\hline Royal Hospital for Women & 3077 & 73.2 & 383 & 9.1 & 368 & 8.8 & 277 & 6.6 & 85 & 2.0 & 13 & 0.3 & 0 & 0.0 & 4203 & 100.0 \\
\hline Liverpool & 2532 & 80.6 & 221 & 7.0 & 162 & 5.2 & 187 & 6.0 & 30 & 1.0 & 8 & 0.3 & 1 & 0.0 & 3141 & 100.0 \\
\hline Nepean & 2467 & 67.5 & 564 & 15.4 & 343 & 9.4 & 201 & 5.5 & 57 & 1.6 & 21 & 0.6 & 0 & 0.0 & 3653 & 100.0 \\
\hline Westmead & 3343 & 70.8 & 404 & 8.6 & 539 & 11.4 & 275 & 5.8 & 150 & 3.2 & 13 & 0.3 & 0 & 0.0 & 4724 & 100.0 \\
\hline John Hunter & 2691 & 71.0 & 124 & 3.3 & 312 & 8.2 & 592 & 15.6 & 41 & 1.1 & 32 & 0.8 & 0 & 0.0 & 3792 & 100.0 \\
\hline TOTAL & 19452 & 72.8 & 2226 & 8.3 & 2202 & 8.2 & 2257 & 8.4 & 458 & 1.7 & 134 & 0.5 & 1 & 0.0 & 26730 & 100.0 \\
\hline \multicolumn{17}{|l|}{ Level 5} \\
\hline Gosford & 1879 & 72.4 & 163 & 6.3 & 297 & 11.4 & 227 & 8.8 & 12 & 0.5 & 16 & 0.6 & 0 & 0.0 & 2594 & 100.0 \\
\hline St. George & 1992 & 77.9 & 101 & 4.0 & 282 & 11.0 & 173 & 6.8 & 3 & 0.1 & 5 & 0.2 & 0 & 0.0 & 2556 & 100.0 \\
\hline Blacktown & 2317 & 71.6 & 500 & 15.5 & 247 & 7.6 & 139 & 4.3 & 11 & 0.3 & 22 & 0.7 & 0 & 0.0 & 3236 & 100.0 \\
\hline Lismore Base & 1029 & 76.7 & 50 & 3.7 & 170 & 12.7 & 88 & 6.6 & 1 & 0.1 & 3 & 0.2 & 0 & 0.0 & 1341 & 100.0 \\
\hline Tweed Heads & 1090 & 71.9 & 30 & 2.0 & 249 & 16.4 & 135 & 8.9 & 7 & 0.5 & 4 & 0.3 & 0 & 0.0 & 1515 & 100.0 \\
\hline Port Macquarie Base & 617 & 77.4 & 17 & 2.1 & 105 & 13.2 & 51 & 6.4 & 3 & 0.4 & 4 & 0.5 & 0 & 0.0 & 797 & 100.0 \\
\hline Wollongong & 1659 & 70.1 & 359 & 15.2 & 219 & 9.3 & 107 & 4.5 & 8 & 0.3 & 14 & 0.6 & 0 & 0.0 & 2366 & 100.0 \\
\hline Maitland & 1245 & 80.5 & 13 & 0.8 & 145 & 9.4 & 132 & 8.5 & 3 & 0.2 & 8 & 0.5 & 0 & 0.0 & 1546 & 100.0 \\
\hline Wagga Wagga Base & 600 & 78.4 & 40 & 5.2 & 58 & 7.6 & 62 & 8.1 & 3 & 0.4 & 2 & 0.3 & 0 & 0.0 & 765 & 100.0 \\
\hline TOTAL & 12428 & 74.3 & 1273 & 7.6 & 1772 & 10.6 & 1114 & 6.7 & 51 & 0.3 & 78 & 0.5 & 0 & 0.0 & 16716 & 100.0 \\
\hline \multicolumn{17}{|l|}{ Level 4} \\
\hline Canterbury & 1466 & 86.1 & 48 & 2.8 & 142 & 8.3 & 35 & 2.1 & 2 & 0.1 & 9 & 0.5 & 0 & 0.0 & 1702 & 100.0 \\
\hline Hornsby & 909 & 77.9 & 41 & 3.5 & 134 & 11.5 & 78 & 6.7 & 2 & 0.2 & 3 & 0.3 & 0 & 0.0 & 1167 & 100.0 \\
\hline Manly & 872 & 76.2 & 37 & 3.2 & 161 & 14.1 & 71 & 6.2 & 2 & 0.2 & 1 & 0.1 & 0 & 0.0 & 1144 & 100.0 \\
\hline
\end{tabular}


Table 46. (Continued)

\begin{tabular}{|c|c|c|c|c|c|c|c|c|c|c|c|c|c|c|c|c|}
\hline \multirow{3}{*}{$\begin{array}{l}\text { Maternity service level - } \\
\text { hospital }\end{array}$} & \multicolumn{16}{|c|}{ Resuscitation } \\
\hline & \multicolumn{2}{|c|}{ None } & \multicolumn{2}{|c|}{ Suction } & \multicolumn{2}{|c|}{$\begin{array}{l}\text { Oxygen } \\
\text { therapy }\end{array}$} & \multicolumn{2}{|c|}{$\begin{array}{l}\text { IPPR by bag } \\
\text { and mask }\end{array}$} & \multicolumn{2}{|c|}{$\begin{array}{l}\text { Intubation } \\
\text { and IPPR }\end{array}$} & \multicolumn{2}{|c|}{$\begin{array}{c}\text { External cardiac } \\
\text { massage and } \\
\text { ventilation }\end{array}$} & \multicolumn{2}{|c|}{ Not stated } & \multicolumn{2}{|c|}{ TOTAL } \\
\hline & No. & $\%$ & No. & $\%$ & No. & $\%$ & No. & $\%$ & No. & $\%$ & No. & $\%$ & No. & $\%$ & No. & $\%$ \\
\hline Mona Vale & 180 & 57.0 & 87 & 27.5 & 36 & 11.4 & 13 & 4.1 & 0 & 0.0 & 0 & 0 & 0 & 0.0 & 316 & 100.0 \\
\hline Sutherland & 1077 & 84.0 & 90 & 7.0 & 68 & 5.3 & 42 & 3.3 & 2 & 0.2 & 3 & 0.2 & 0 & 0.0 & 1282 & 100.0 \\
\hline Auburn & 1148 & 85.4 & 44 & 3.3 & 91 & 6.8 & 58 & 4.3 & 0 & 0.0 & 3 & 0.2 & 0 & 0.0 & 1344 & 100.0 \\
\hline Fairfield & 1682 & 88.8 & 55 & 2.9 & 74 & 3.9 & 74 & 3.9 & 3 & 0.2 & 6 & 0.3 & 0 & 0.0 & 1894 & 100.0 \\
\hline Campbelltown & 2289 & 84.5 & 80 & 3.0 & 194 & 7.2 & 125 & 4.6 & 7 & 0.3 & 15 & 0.6 & 0 & 0.0 & 2710 & 100.0 \\
\hline Bankstown-Lidcombe & 1887 & 86.0 & 114 & 5.2 & 109 & 5.0 & 66 & 3.0 & 2 & 0.1 & 16 & 0.7 & 0 & 0.0 & 2194 & 100.0 \\
\hline Hawkesbury & 677 & 78.0 & 54 & 6.2 & 113 & 13.0 & 20 & 2.3 & 3 & 0.3 & 1 & 0.1 & 0 & 0.0 & 868 & 100.0 \\
\hline Coffs Harbour & 859 & 79.1 & 69 & 6.4 & 100 & 9.2 & 54 & 5.0 & 2 & 0.2 & 2 & 0.2 & 0 & 0.0 & 1086 & 100.0 \\
\hline Grafton Base & 326 & 66.0 & 101 & 20.4 & 32 & 6.5 & 31 & 6.3 & 4 & 0.8 & 0 & 0.0 & 0 & 0.0 & 494 & 100.0 \\
\hline Tamworth Base & 586 & 69.4 & 46 & 5.5 & 115 & 13.6 & 85 & 10.1 & 9 & 1.1 & 3 & 0.4 & 0 & 0.0 & 844 & 100.0 \\
\hline Manning Base & 617 & 81.2 & 14 & 1.8 & 79 & 10.4 & 49 & 6.4 & 1 & 0.1 & 0 & 0.0 & 0 & 0.0 & 760 & 100.0 \\
\hline Dubbo Base & 949 & 77.7 & 42 & 3.4 & 143 & 11.7 & 80 & 6.6 & 3 & 0.2 & 4 & 0.3 & 0 & 0.0 & 1221 & 100.0 \\
\hline Orange Base & 681 & 75.4 & 20 & 2.2 & 132 & 14.6 & 62 & 6.9 & 4 & 0.4 & 4 & 0.4 & 0 & 0.0 & 903 & 100.0 \\
\hline Goulburn Base & 225 & 74.5 & 18 & 6.0 & 32 & 10.6 & 22 & 7.3 & 1 & 0.3 & 4 & 1.3 & 0 & 0.0 & 302 & 100.0 \\
\hline Shoalhaven & 711 & 80.2 & 70 & 7.9 & 69 & 7.8 & 35 & 3.9 & 0 & 0.0 & 2 & 0.2 & 0 & 0.0 & 887 & 100.0 \\
\hline Griffith Base & 305 & 73.5 & 21 & 5.1 & 59 & 14.2 & 26 & 6.3 & 2 & 0.5 & 2 & 0.5 & 0 & 0.0 & 415 & 100.0 \\
\hline TOTAL & 17446 & 81.0 & 1051 & 4.9 & 1883 & 8.7 & 1026 & 4.8 & 49 & 0.2 & 78 & 0.4 & 0 & 0.0 & 21533 & 100.0 \\
\hline \multicolumn{17}{|l|}{ Level 3} \\
\hline Blue Mountains & 181 & 73.6 & 18 & 7.3 & 33 & 13.4 & 13 & 5.3 & 0 & 0.0 & 1 & 0.4 & 0 & 0.0 & 246 & 100.0 \\
\hline Kempsey & 243 & 79.2 & 3 & 1.0 & 23 & 7.5 & 36 & 11.7 & 0 & 0.0 & 2 & 0.7 & 0 & 0.0 & 307 & 100.0 \\
\hline Murwillumbah & 148 & 70.8 & 24 & 11.5 & 30 & 14.4 & 7 & 3.3 & 0 & 0.0 & 0 & 0.0 & 0 & 0.0 & 209 & 100.0 \\
\hline Armidale & 326 & 72.9 & 11 & 2.5 & 94 & 21.0 & 16 & 3.6 & 0 & 0.0 & 0 & 0.0 & 0 & 0.0 & 447 & 100.0 \\
\hline Inverell & 66 & 25.7 & 12 & 4.7 & 119 & 46.3 & 58 & 22.6 & 2 & 0.8 & 0 & 0.0 & 0 & 0.0 & 257 & 100.0 \\
\hline Mudgee & 188 & 82.1 & 9 & 3.9 & 14 & 6.1 & 17 & 7.4 & 1 & 0.4 & 0 & 0.0 & 0 & 0.0 & 229 & 100.0 \\
\hline Bathurst Base & 444 & 78.4 & 36 & 6.4 & 50 & 8.8 & 31 & 5.5 & 4 & 0.7 & 1 & 0.2 & 0 & 0.0 & 566 & 100.0 \\
\hline Bega & 243 & 86.5 & 4 & 1.4 & 16 & 5.7 & 18 & 6.4 & 0 & 0.0 & 0 & 0.0 & 0 & 0.0 & 281 & 100.0 \\
\hline Moruya & 282 & 86.8 & 10 & 3.1 & 18 & 5.5 & 15 & 4.6 & 0 & 0.0 & 0 & 0.0 & 0 & 0.0 & 325 & 100.0 \\
\hline Queanbeyan & 281 & 90.9 & 4 & 1.3 & 13 & 4.2 & 10 & 3.2 & 1 & 0.3 & 0 & 0.0 & 0 & 0.0 & 309 & 100.0 \\
\hline Bowral & 477 & 83.8 & 28 & 4.9 & 46 & 8.1 & 13 & 2.3 & 2 & 0.4 & 3 & 0.5 & 0 & 0.0 & 569 & 100.0 \\
\hline Broken Hill Base & 177 & 73.1 & 25 & 10.3 & 27 & 11.2 & 12 & 5.0 & 0 & 0.0 & 1 & 0.4 & 0 & 0.0 & 242 & 100.0 \\
\hline Other hospitals & 1833 & 72.0 & 210 & 8.3 & 380 & 14.9 & 113 & 4.4 & 2 & 0.1 & 7 & 0.3 & 0 & 0.0 & 2545 & 100.0 \\
\hline TOTAL & 4889 & 74.8 & 394 & 6.0 & 863 & 13.2 & 359 & 5.5 & 12 & 0.2 & 15 & 0.2 & 0 & 0.0 & 6532 & 100.0 \\
\hline \multicolumn{17}{|l|}{ Level 2} \\
\hline Wyong & 201 & 85.9 & 4 & 1.7 & 16 & 6.8 & 12 & 5.1 & 0 & 0.0 & 1 & 0.4 & 0 & 0.0 & 234 & 100.0 \\
\hline Other hospitals & 722 & 84.5 & 17 & 2.0 & 53 & 6.2 & 62 & 7.3 & 0 & 0.0 & 0 & 0.0 & 0 & 0.0 & 854 & 100.0 \\
\hline TOTAL & 923 & 84.8 & 21 & 1.9 & 69 & 6.3 & 74 & 6.8 & 0 & 0.0 & 1 & 0.1 & 0 & 0.0 & 1088 & 100.0 \\
\hline Private & & & & & & & & & & & & & & & & \\
\hline Mater, North Sydney & 1795 & 70.8 & 166 & 6.5 & 421 & 16.6 & 152 & 6.0 & 1 & 0.0 & 1 & 0.0 & 0 & 0.0 & 2536 & 100.0 \\
\hline North Shore Private & 2066 & 77.3 & 262 & 9.8 & 256 & 9.6 & 83 & 3.1 & 4 & 0.1 & 0 & 0.0 & 0 & 0.0 & 2671 & 100.0 \\
\hline Sydney Adventist & 1910 & 83.6 & 120 & 5.2 & 166 & 7.3 & 84 & 3.7 & 0 & 0.0 & 1 & 0.0 & 5 & 0.2 & 2286 & 100.0 \\
\hline North Gosford Private & 614 & 72.4 & 73 & 8.6 & 135 & 15.9 & 25 & 2.9 & 0 & 0.0 & 1 & 0.1 & 0 & 0.0 & 848 & 100.0 \\
\hline Hurstville Private & 275 & 28.2 & 440 & 45.1 & 196 & 20.1 & 62 & 6.4 & 2 & 0.2 & 0 & 0.0 & 0 & 0.0 & 975 & 100.0 \\
\hline Kareena Private & 383 & 55.6 & 204 & 29.6 & 74 & 10.7 & 26 & 3.8 & 2 & 0.3 & 0 & 0.0 & 0 & 0.0 & 689 & 100.0 \\
\hline St. George Private & 717 & 38.9 & 775 & 42.0 & 285 & 15.4 & 64 & 3.5 & 2 & 0.1 & 2 & 0.1 & 0 & 0.0 & 1845 & 100.0 \\
\hline Prince of Wales Private & 1261 & 55.0 & 469 & 20.5 & 468 & 20.4 & 90 & 3.9 & 0 & 0.0 & 3 & 0.1 & 0 & 0.0 & 2291 & 100.0 \\
\hline Norwest Private & 1077 & 86.8 & 44 & 3.5 & 83 & 6.7 & 35 & 2.8 & 1 & 0.1 & 1 & 0.1 & 0 & 0.0 & 1241 & 100.0 \\
\hline Sydney Southwest Private & 781 & 73.1 & 148 & 13.8 & 98 & 9.2 & 41 & 3.8 & 0 & 0.0 & 1 & 0.1 & 0 & 0.0 & 1069 & 100.0 \\
\hline Nepean Private & 618 & 65.7 & 87 & 9.3 & 184 & 19.6 & 41 & 4.4 & 8 & 0.9 & 2 & 0.2 & 0 & 0.0 & 940 & 100.0 \\
\hline Westmead Private & 1946 & 81.3 & 199 & 8.3 & 152 & 6.4 & 93 & 3.9 & 0 & 0.0 & 3 & 0.1 & 0 & 0.0 & 2393 & 100.0 \\
\hline Figtree Private & 318 & 30.8 & 591 & 57.2 & 105 & 10.2 & 17 & 1.6 & 1 & 0.1 & 0 & 0.0 & 1 & 0.1 & 1033 & 100.0 \\
\hline Newcastle Private & 1547 & 81.3 & 74 & 3.9 & 166 & 8.7 & 111 & 5.8 & 4 & 0.2 & 1 & 0.1 & 0 & 0.0 & 1903 & 100.0 \\
\hline Calvary, Wagga Wagga & 434 & 67.9 & 95 & 14.9 & 66 & 10.3 & 44 & 6.9 & 0 & 0.0 & 0 & 0.0 & 0 & 0.0 & 639 & 100.0 \\
\hline Other hospitals & 189 & 69.7 & 27 & 10.0 & 43 & 15.9 & 10 & 3.7 & 2 & 0.7 & 0 & 0.0 & 0 & 0.0 & 271 & 100.0 \\
\hline TOTAL & 15931 & 67.4 & 3774 & 16.0 & 2898 & 12.3 & 978 & 4.1 & 27 & 0.1 & 16 & 0.1 & 6 & 0.0 & 23630 & 100.0 \\
\hline TOTAL NSW* & 71257 & 73.9 & 8746 & 9.1 & 9698 & 10.1 & 5812 & 6.0 & 597 & 0.6 & 322 & 0.3 & 7 & 0.0 & 96439 & 100.0 \\
\hline
\end{tabular}




\section{Admission to special care}

\section{or neonatal intensive care}

Tables 47 shows admissions of live born babies to special care or neonatal intensive care for Local Health Districts, and Table

48 for individual hospitals by maternity service level where at least 200 mothers gave birth in 2009 .

\section{Table 47. Live births by admission to special care or neonatal intensive care by Local Health District of hospital, NSW 2009}

\begin{tabular}{|c|c|c|c|c|c|c|c|c|}
\hline \multirow[t]{3}{*}{ Local Health District } & \multicolumn{8}{|c|}{ Admission to special care or NICU } \\
\hline & \multicolumn{2}{|c|}{ No } & \multicolumn{2}{|c|}{ Yes } & \multicolumn{2}{|c|}{ Not stated } & \multicolumn{2}{|c|}{ Total } \\
\hline & No. & $\%$ & No. & $\%$ & No. & $\%$ & No. & $\%$ \\
\hline Sydney & 5924 & 87.7 & 831 & 12.3 & 0 & 0.0 & 6755 & 100.0 \\
\hline South Western Sydney & 9257 & 88.7 & 1175 & 11.3 & 0 & 0.0 & 10432 & 100.0 \\
\hline South Eastern Sydney & 6574 & 82.4 & 1402 & 17.6 & 0 & 0.0 & 7976 & 100.0 \\
\hline Illawarra Shoalhaven & 2737 & 82.3 & 588 & 17.7 & 0 & 0.0 & 3325 & 100.0 \\
\hline Western Sydney & 7363 & 79.8 & 1860 & 20.2 & 0 & 0.0 & 9223 & 100.0 \\
\hline Nepean Blue Mountains & 3741 & 75.9 & 1185 & 24.1 & 0 & 0.0 & 4926 & 100.0 \\
\hline Northern Sydney & 4013 & 83.2 & 813 & 16.8 & 0 & 0.0 & 4826 & 100.0 \\
\hline Central Coast & 2345 & 83.4 & 468 & 16.6 & 0 & 0.0 & 2813 & 100.0 \\
\hline Hunter New England & 7271 & 82.5 & 1545 & 17.5 & 0 & 0.0 & 8816 & 100.0 \\
\hline Northern NSW & 3146 & 84.2 & 587 & 15.7 & 2 & 0.1 & 3735 & 100.0 \\
\hline Mid North Coast & 1930 & 86.8 & 294 & 13.2 & 0 & 0.0 & 2224 & 100.0 \\
\hline Southern NSW & 1329 & 94.7 & 74 & 5.3 & 0 & 0.0 & 1403 & 100.0 \\
\hline Murrumbidgee & 1685 & 85.0 & 297 & 15.0 & 0 & 0.0 & 1982 & 100.0 \\
\hline Western NSW & 3030 & 87.9 & 419 & 12.1 & 0 & 0.0 & 3449 & 100.0 \\
\hline Far West & 223 & 92.5 & 18 & 7.5 & 0 & 0.0 & 241 & 100.0 \\
\hline Private Hospitals & 21456 & 91.1 & 2091 & 8.9 & 2 & 0.0 & 23549 & 100.0 \\
\hline TOTAL* & 82184 & 85.8 & 13649 & 14.2 & 4 & 0.0 & 95837 & 100.0 \\
\hline
\end{tabular}

Source: NSW Perinatal Data Collection (HOIST). Centre for Epidemiology and Research, NSW Ministry of Health.

* Total includes live births at home assisted by independent midwives.

NICU: neonatal intensive care unit.

Table 48. Live births by admission to special care or neonatal intensive care, hospital and maternity service level, NSW 2009\#

\begin{tabular}{|c|c|c|c|c|c|c|c|c|}
\hline \multirow[t]{3}{*}{ Maternity service level-hospital } & \multicolumn{8}{|c|}{ Admission to Special Care or NICU } \\
\hline & \multicolumn{2}{|c|}{ No } & \multicolumn{2}{|c|}{ Yes } & \multicolumn{2}{|c|}{ Not stated } & \multicolumn{2}{|c|}{ TOTAL } \\
\hline & No. & $\%$ & No. & $\%$ & No. & $\%$ & No. & $\%$ \\
\hline \multicolumn{9}{|l|}{ Level 6} \\
\hline Royal Prince Alfred & 4378 & 86.4 & 687 & 13.6 & 0 & 0.0 & 5065 & 100.0 \\
\hline Royal North Shore & 1814 & 87.0 & 271 & 13.0 & 0 & 0.0 & 2085 & 100.0 \\
\hline Royal Hospital for Women & 3393 & 81.7 & 761 & 18.3 & 0 & 0.0 & 4154 & 100.0 \\
\hline Liverpool & 2573 & 82.6 & 541 & 17.4 & 0 & 0.0 & 3114 & 100.0 \\
\hline Nepean & 2621 & 72.3 & 1002 & 27.7 & 0 & 0.0 & 3623 & 100.0 \\
\hline Westmead & 3664 & 78.5 & 1001 & 21.5 & 0 & 0.0 & 4665 & 100.0 \\
\hline John Hunter & 2994 & 79.7 & 762 & 20.3 & 0 & 0.0 & 3756 & 100.0 \\
\hline TOTAL & 21437 & 81.0 & 5025 & 19.0 & 0 & 0.0 & 26462 & 100.0 \\
\hline \multicolumn{9}{|l|}{ Level 5} \\
\hline Gosford & 2115 & 82.0 & 464 & 18.0 & 0 & 0.0 & 2579 & 100.0 \\
\hline St. George & 2100 & 82.5 & 444 & 17.5 & 0 & 0.0 & 2544 & 100.0 \\
\hline Blacktown & 2628 & 81.6 & 594 & 18.4 & 0 & 0.0 & 3222 & 100.0 \\
\hline Lismore Base & 1057 & 79.2 & 277 & 20.8 & 0 & 0.0 & 1334 & 100.0 \\
\hline Tweed Heads & 1261 & 84.0 & 241 & 16.0 & 0 & 0.0 & 1502 & 100.0 \\
\hline Port Macquarie Base & 697 & 88.2 & 93 & 11.8 & 0 & 0.0 & 790 & 100.0 \\
\hline Wollongong & 1855 & 78.8 & 499 & 21.2 & 0 & 0.0 & 2354 & 100.0 \\
\hline Maitland & 1281 & 83.7 & 249 & 16.3 & 0 & 0.0 & 1530 & 100.0 \\
\hline Wagga Wagga Base & 615 & 81.3 & 141 & 18.7 & 0 & 0.0 & 756 & 100.0 \\
\hline TOTAL & 13609 & 81.9 & 3002 & 18.1 & 0 & 0.0 & 16611 & 100.0 \\
\hline \multicolumn{9}{|l|}{ Level 4} \\
\hline Canterbury & 1546 & 91.5 & 144 & 8.5 & 0 & 0.0 & 1690 & 100.0 \\
\hline Hornsby & 894 & 76.8 & 270 & 23.2 & 0 & 0.0 & 1164 & 100.0 \\
\hline Manly & 906 & 79.4 & 235 & 20.6 & 0 & 0.0 & 1141 & 100.0 \\
\hline
\end{tabular}


Table 48. (Continued)

\begin{tabular}{|c|c|c|c|c|c|c|c|c|}
\hline \multirow[t]{3}{*}{ Maternity service level-hospital } & \multicolumn{8}{|c|}{ Admission to Special Care or NICU } \\
\hline & \multicolumn{2}{|c|}{ No } & \multicolumn{2}{|c|}{ Yes } & \multicolumn{2}{|c|}{ Not stated } & \multicolumn{2}{|c|}{ TOTAL } \\
\hline & No. & $\%$ & No. & $\%$ & No. & $\%$ & No. & $\%$ \\
\hline Mona Vale & 280 & 88.9 & 35 & 11.1 & 0 & 0.0 & 315 & 100.0 \\
\hline Sutherland & 1081 & 84.6 & 197 & 15.4 & 0 & 0.0 & 1278 & 100.0 \\
\hline Auburn & 1071 & 80.2 & 265 & 19.8 & 0 & 0.0 & 1336 & 100.0 \\
\hline Fairfield & 1670 & 88.8 & 211 & 11.2 & 0 & 0.0 & 1881 & 100.0 \\
\hline Campbelltown & 2552 & 94.8 & 139 & 5.2 & 0 & 0.0 & 2691 & 100.0 \\
\hline Bankstown-Lidcombe & 1922 & 88.3 & 255 & 11.7 & 0 & 0.0 & 2177 & 100.0 \\
\hline Hawkesbury & 717 & 82.7 & 150 & 17.3 & 0 & 0.0 & 867 & 100.0 \\
\hline Coffs Harbour & 903 & 83.6 & 177 & 16.4 & 0 & 0.0 & 1080 & 100.0 \\
\hline Grafton Base & 439 & 89.2 & 53 & 10.8 & 0 & 0.0 & 492 & 100.0 \\
\hline Tamworth Base & 554 & 66.0 & 285 & 34.0 & 0 & 0.0 & 839 & 100.0 \\
\hline Manning Base & 616 & 81.8 & 137 & 18.2 & 0 & 0.0 & 753 & 100.0 \\
\hline Dubbo Base & 1028 & 84.5 & 189 & 15.5 & 0 & 0.0 & 1217 & 100.0 \\
\hline Orange Base & 740 & 82.5 & 157 & 17.5 & 0 & 0.0 & 897 & 100.0 \\
\hline Goulburn Base & 272 & 90.7 & 28 & 9.3 & 0 & 0.0 & 300 & 100.0 \\
\hline Shoalhaven & 794 & 90.2 & 86 & 9.8 & 0 & 0.0 & 880 & 100.0 \\
\hline Griffith Base & 289 & 70.0 & 124 & 30.0 & 0 & 0.0 & 413 & 100.0 \\
\hline TOTAL & 18274 & 85.3 & 3137 & 14.7 & 0 & 0.0 & 21411 & 100.0 \\
\hline \multicolumn{9}{|l|}{ Level 3} \\
\hline Blue Mountains & 222 & 90.2 & 24 & 9.8 & 0 & 0.0 & 246 & 100.0 \\
\hline Kempsey & 281 & 92.4 & 23 & 7.6 & 0 & 0.0 & 304 & 100.0 \\
\hline Murwillumbah & 198 & 94.7 & 11 & 5.3 & 0 & 0.0 & 209 & 100.0 \\
\hline Armidale & 404 & 90.6 & 42 & 9.4 & 0 & 0.0 & 446 & 100.0 \\
\hline Inverell & 229 & 89.8 & 26 & 10.2 & 0 & 0.0 & 255 & 100.0 \\
\hline Mudgee & 222 & 97.8 & 5 & 2.2 & 0 & 0.0 & 227 & 100.0 \\
\hline Bathurst Base & 522 & 92.4 & 43 & 7.6 & 0 & 0.0 & 565 & 100.0 \\
\hline Bega & 258 & 92.1 & 22 & 7.9 & 0 & 0.0 & 280 & 100.0 \\
\hline Moruya & 305 & 95.0 & 16 & 5.0 & 0 & 0.0 & 321 & 100.0 \\
\hline Queanbeyan & 307 & 99.4 & 2 & 0.6 & 0 & 0.0 & 309 & 100.0 \\
\hline Bowral & 539 & 94.9 & 29 & 5.1 & 0 & 0.0 & 568 & 100.0 \\
\hline Broken Hill Base & 223 & 92.5 & 18 & 7.5 & 0 & 0.0 & 241 & 100.0 \\
\hline Other hospitals & 2439 & 96.0 & 101 & 4.0 & 1 & 0.0 & 2541 & 100.0 \\
\hline TOTAL & 6149 & 94.4 & 362 & 5.6 & 1 & 0.0 & 6512 & 100.0 \\
\hline \multicolumn{9}{|l|}{ Level 2} \\
\hline Wyong & 230 & 98.3 & 4 & 1.7 & 0 & 0.0 & 234 & 100.0 \\
\hline Other hospitals & 828 & 97.2 & 23 & 2.7 & 1 & 0.1 & 852 & 100.0 \\
\hline TOTAL & 1058 & 97.4 & 27 & 2.5 & 1 & 0.1 & 1086 & 100.0 \\
\hline \multicolumn{9}{|l|}{ Private } \\
\hline Mater, North Sydney & 2272 & 89.7 & 260 & 10.3 & 0 & 0.0 & 2532 & 100.0 \\
\hline North Shore Private & 2650 & 99.6 & 11 & 0.4 & 0 & 0.0 & 2661 & 100.0 \\
\hline Sydney Adventist & 1936 & 85.2 & 337 & 14.8 & 0 & 0.0 & 2273 & 100.0 \\
\hline North Gosford Private & 732 & 86.5 & 114 & 13.5 & 0 & 0.0 & 846 & 100.0 \\
\hline Hurstville Private & 825 & 84.7 & 149 & 15.3 & 0 & 0.0 & 974 & 100.0 \\
\hline Kareena Private & 684 & 99.6 & 3 & 0.4 & 0 & 0.0 & 687 & 100.0 \\
\hline St. George Private & 1836 & 99.7 & 5 & 0.3 & 0 & 0.0 & 1841 & 100.0 \\
\hline Prince of Wales Private & 2042 & 89.4 & 243 & 10.6 & 0 & 0.0 & 2285 & 100.0 \\
\hline Norwest Private & 1065 & 86.3 & 169 & 13.7 & 0 & 0.0 & 1234 & 100.0 \\
\hline Sydney Southwest Private & 825 & 77.2 & 243 & 22.8 & 0 & 0.0 & 1068 & 100.0 \\
\hline Nepean Private & 694 & 74.0 & 244 & 26.0 & 0 & 0.0 & 938 & 100.0 \\
\hline Westmead Private & 2368 & 99.5 & 12 & 0.5 & 0 & 0.0 & 2380 & 100.0 \\
\hline Figtree Private & 1025 & 99.9 & 1 & 0.1 & 0 & 0.0 & 1026 & 100.0 \\
\hline Newcastle Private & 1655 & 87.3 & 241 & 12.7 & 0 & 0.0 & 1896 & 100.0 \\
\hline Calvary, Wagga Wagga & 581 & 91.2 & 56 & 8.8 & 0 & 0.0 & 637 & 100.0 \\
\hline Other hospitals & 266 & 98.2 & 3 & 1.1 & 2 & 0.7 & 271 & 100.0 \\
\hline TOTAL & 21456 & 91.1 & 2091 & 8.9 & 2 & 0.0 & 23549 & 100.0 \\
\hline TOTAL* & 82184 & 85.8 & 13649 & 14.2 & 4 & 0.0 & 95837 & 100.0 \\
\hline
\end{tabular}




\section{Infant feeding}

Table 49 shows infant feeding at discharge from the hospital of birth for Local Health Districts, and Table 50 for individual hospitals by maternity service level where at least 200 mothers gave birth in 2009 .

Table 49. Live births by type of infant feeding and Local Health District of hospital, NSW 2009

\begin{tabular}{|c|c|c|c|c|c|c|c|c|c|c|}
\hline \multirow[t]{3}{*}{ Local Health District } & \multicolumn{10}{|c|}{ Infant feeding } \\
\hline & \multicolumn{2}{|c|}{ Full breast-feeding } & \multicolumn{2}{|c|}{$\begin{array}{l}\text { Any breast- } \\
\text { feeding }\end{array}$} & \multicolumn{2}{|c|}{$\begin{array}{c}\text { Infant formula } \\
\text { only }\end{array}$} & \multicolumn{2}{|c|}{ Not stated } & \multicolumn{2}{|c|}{ Total } \\
\hline & No. & $\%$ & No. & $\%$ & No. & $\%$ & No. & $\%$ & No. & $\%$ \\
\hline Sydney & 5524 & 81.8 & 765 & 11.3 & 410 & 6.1 & 56 & 0.8 & 6755 & 100.0 \\
\hline South Western Sydney & 7331 & 70.3 & 1121 & 10.7 & 1925 & 18.5 & 55 & 0.5 & 10432 & 100.0 \\
\hline South Eastern Sydney & 6674 & 83.7 & 665 & 8.3 & 566 & 7.1 & 71 & 0.9 & 7976 & 100.0 \\
\hline Illawarra Shoalhaven & 2561 & 77.0 & 77 & 2.3 & 645 & 19.4 & 42 & 1.3 & 3325 & 100.0 \\
\hline Western Sydney & 7346 & 79.6 & 680 & 7.4 & 1022 & 11.1 & 175 & 1.9 & 9223 & 100.0 \\
\hline Nepean Blue Mountains & 3612 & 73.3 & 271 & 5.5 & 1004 & 20.4 & 39 & 0.8 & 4926 & 100.0 \\
\hline Northern Sydney & 4286 & 88.8 & 290 & 6.0 & 217 & 4.5 & 33 & 0.7 & 4826 & 100.0 \\
\hline Central Coast & 2269 & 80.7 & 85 & 3.0 & 419 & 14.9 & 40 & 1.4 & 2813 & 100.0 \\
\hline Hunter New England & 6992 & 79.3 & 307 & 3.5 & 1445 & 16.4 & 72 & 0.8 & 8816 & 100.0 \\
\hline Northern NSW & 3322 & 88.9 & 115 & 3.1 & 286 & 7.7 & 12 & 0.3 & 3735 & 100.0 \\
\hline Mid North Coast & 1942 & 87.3 & 63 & 2.8 & 212 & 9.5 & 7 & 0.3 & 2224 & 100.0 \\
\hline Southern NSW & 1214 & 86.5 & 31 & 2.2 & 154 & 11.0 & 4 & 0.3 & 1403 & 100.0 \\
\hline Murrumbidgee & 1506 & 76.0 & 45 & 2.3 & 425 & 21.4 & 6 & 0.3 & 1982 & 100.0 \\
\hline Western NSW & 2770 & 80.3 & 64 & 1.9 & 606 & 17.6 & 9 & 0.3 & 3449 & 100.0 \\
\hline Far West & 183 & 75.9 & 12 & 5.0 & 46 & 19.1 & 0 & 0.0 & 241 & 100.0 \\
\hline Private Hospitals & 19499 & 82.8 & 1831 & 7.8 & 2177 & 9.2 & 42 & 0.2 & 23549 & 100.0 \\
\hline TOTAL* & 77155 & 80.5 & 6423 & 6.7 & 11559 & 12.1 & 700 & 0.7 & 95837 & 100.0 \\
\hline
\end{tabular}

Table 50. Live births by type of infant feeding, hospital and maternity service level, NSW 2009\#

\begin{tabular}{|c|c|c|c|c|c|c|c|c|c|c|}
\hline \multirow[t]{3}{*}{ Maternity service level-hospital } & \multicolumn{10}{|c|}{ Infant feeding } \\
\hline & \multicolumn{2}{|c|}{ Full breast-feeding } & \multicolumn{2}{|c|}{$\begin{array}{l}\text { Any breast- } \\
\text { feeding }\end{array}$} & \multicolumn{2}{|c|}{$\begin{array}{c}\text { Infant formula } \\
\text { only }\end{array}$} & \multicolumn{2}{|c|}{ Not stated } & \multicolumn{2}{|c|}{ Total } \\
\hline & No. & $\%$ & No. & $\%$ & No. & $\%$ & No. & $\%$ & No. & $\%$ \\
\hline \multicolumn{11}{|l|}{ Level 6} \\
\hline Royal Prince Alfred & 4162 & 82.2 & 604 & 11.9 & 255 & 5.0 & 44 & 0.9 & 5065 & 100.0 \\
\hline Royal North Shore & 1795 & 86.1 & 163 & 7.8 & 116 & 5.6 & 11 & 0.5 & 2085 & 100.0 \\
\hline Royal Hospital for Women & 3578 & 86.1 & 309 & 7.4 & 226 & 5.4 & 41 & 1.0 & 4154 & 100.0 \\
\hline Liverpool & 2009 & 64.5 & 470 & 15.1 & 605 & 19.4 & 30 & 1.0 & 3114 & 100.0 \\
\hline Nepean & 2595 & 71.6 & 229 & 6.3 & 777 & 21.4 & 22 & 0.6 & 3623 & 100.0 \\
\hline Westmead & 3694 & 79.2 & 412 & 8.8 & 442 & 9.5 & 117 & 2.5 & 4665 & 100.0 \\
\hline John Hunter & 2934 & 78.1 & 140 & 3.7 & 640 & 17.0 & 42 & 1.1 & 3756 & 100.0 \\
\hline Total & 20767 & 78.5 & 2327 & 8.8 & 3061 & 11.6 & 307 & 1.2 & 26462 & 100.0 \\
\hline \multicolumn{11}{|l|}{ Level 5} \\
\hline Gosford & 2061 & 79.9 & 84 & 3.3 & 395 & 15.3 & 39 & 1.5 & 2579 & 100.0 \\
\hline St. George & 1952 & 76.7 & 315 & 12.4 & 254 & 10.0 & 23 & 0.9 & 2544 & 100.0 \\
\hline Blacktown & 2492 & 77.3 & 202 & 6.3 & 487 & 15.1 & 41 & 1.3 & 3222 & 100.0 \\
\hline Lismore Base & 1193 & 89.4 & 60 & 4.5 & 74 & 5.5 & 7 & 0.5 & 1334 & 100.0 \\
\hline Tweed Heads & 1372 & 91.3 & 22 & 1.5 & 104 & 6.9 & 4 & 0.3 & 1502 & 100.0 \\
\hline Port Macquarie Base & 689 & 87.2 & 13 & 1.6 & 83 & 10.5 & 5 & 0.6 & 790 & 100.0 \\
\hline Wollongong & 1763 & 74.9 & 53 & 2.3 & 507 & 21.5 & 31 & 1.3 & 2354 & 100.0 \\
\hline Maitland & 1143 & 74.7 & 72 & 4.7 & 309 & 20.2 & 6 & 0.4 & 1530 & 100.0 \\
\hline Wagga Wagga Base & 566 & 74.9 & 13 & 1.7 & 173 & 22.9 & 4 & 0.5 & 756 & 100.0 \\
\hline TOTAL & 13231 & 79.7 & 834 & 5.0 & 2386 & 14.4 & 160 & 1.0 & 16611 & 100.0 \\
\hline \multicolumn{11}{|l|}{ Level 4} \\
\hline Canterbury & 1362 & 80.6 & 161 & 9.5 & 155 & 9.2 & 12 & 0.7 & 1690 & 100.0 \\
\hline Hornsby & 1044 & 89.7 & 67 & 5.8 & 39 & 3.4 & 14 & 1.2 & 1164 & 100.0 \\
\hline Manly & 1044 & 91.5 & 43 & 3.8 & 46 & 4.0 & 8 & 0.7 & 1141 & 100.0 \\
\hline
\end{tabular}


Table 50. (Continued)

\begin{tabular}{|c|c|c|c|c|c|c|c|c|c|c|}
\hline \multirow[t]{3}{*}{ Maternity service level-hospital } & \multicolumn{10}{|c|}{ Infant feeding } \\
\hline & \multicolumn{2}{|c|}{ Full breast-feeding } & \multicolumn{2}{|c|}{$\begin{array}{l}\text { Any breast- } \\
\text { feeding }\end{array}$} & \multicolumn{2}{|c|}{$\begin{array}{c}\text { Infant formula } \\
\text { only }\end{array}$} & \multicolumn{2}{|c|}{ Not stated } & \multicolumn{2}{|c|}{ Total } \\
\hline & No. & $\%$ & No. & $\%$ & No. & $\%$ & No. & $\%$ & No. & $\%$ \\
\hline Mona Vale & 283 & 89.8 & 17 & 5.4 & 15 & 4.8 & 0 & 0.0 & 315 & 100.0 \\
\hline Sutherland & 1144 & 89.5 & 41 & 3.2 & 86 & 6.7 & 7 & 0.5 & 1278 & 100.0 \\
\hline Auburn & 1160 & 86.8 & 66 & 4.9 & 93 & 7.0 & 17 & 1.3 & 1336 & 100.0 \\
\hline Fairfield & 1174 & 62.4 & 347 & 18.4 & 357 & 19.0 & 3 & 0.2 & 1881 & 100.0 \\
\hline Campbelltown & 1932 & 71.8 & 139 & 5.2 & 610 & 22.7 & 10 & 0.4 & 2691 & 100.0 \\
\hline Bankstown-Lidcombe & 1742 & 80.0 & 142 & 6.5 & 284 & 13.0 & 9 & 0.4 & 2177 & 100.0 \\
\hline Hawkesbury & 668 & 77.0 & 24 & 2.8 & 168 & 19.4 & 7 & 0.8 & 867 & 100.0 \\
\hline Coffs Harbour & 942 & 87.2 & 44 & 4.1 & 92 & 8.5 & 2 & 0.2 & 1080 & 100.0 \\
\hline Grafton Base & 376 & 76.4 & 27 & 5.5 & 89 & 18.1 & 0 & 0.0 & 492 & 100.0 \\
\hline Tamworth Base & 668 & 79.6 & 27 & 3.2 & 135 & 16.1 & 9 & 1.1 & 839 & 100.0 \\
\hline Manning Base & 636 & 84.5 & 18 & 2.4 & 98 & 13.0 & 1 & 0.1 & 753 & 100.0 \\
\hline Dubbo Base & 912 & 74.9 & 22 & 1.8 & 282 & 23.2 & 1 & 0.1 & 1217 & 100.0 \\
\hline Orange Base & 747 & 83.3 & 18 & 2.0 & 132 & 14.7 & 0 & 0.0 & 897 & 100.0 \\
\hline Goulburn Base & 236 & 78.7 & 4 & 1.3 & 58 & 19.3 & 2 & 0.7 & 300 & 100.0 \\
\hline Shoalhaven & 715 & 81.3 & 24 & 2.7 & 131 & 14.9 & 10 & 1.1 & 880 & 100.0 \\
\hline Griffith Base & 318 & 77.0 & 13 & 3.1 & 81 & 19.6 & 1 & 0.2 & 413 & 100.0 \\
\hline TOTAL & 17103 & 79.9 & 1244 & 5.8 & 2951 & 13.8 & 113 & 0.5 & 21411 & 100.0 \\
\hline \multicolumn{11}{|l|}{ Level 3} \\
\hline Blue Mountains & 209 & 85.0 & 11 & 4.5 & 22 & 8.9 & 4 & 1.6 & 246 & 100.0 \\
\hline Kempsey & 265 & 87.2 & 5 & 1.6 & 34 & 11.2 & 0 & 0 & 304 & 100.0 \\
\hline Murwillumbah & 192 & 91.9 & 4 & 1.9 & 12 & 5.7 & 1 & 0.5 & 209 & 100.0 \\
\hline Armidale & 387 & 86.8 & 10 & 2.2 & 45 & 10.1 & 4 & 0.9 & 446 & 100.0 \\
\hline Inverell & 194 & 76.1 & 13 & 5.1 & 45 & 17.6 & 3 & 1.2 & 255 & 100.0 \\
\hline Mudgee & 178 & 78.4 & 1 & 0.4 & 48 & 21.1 & 0 & 0.0 & 227 & 100.0 \\
\hline Bathurst Base & 490 & 86.7 & 8 & 1.4 & 62 & 11.0 & 5 & 0.9 & 565 & 100.0 \\
\hline Bega & 245 & 87.5 & 10 & 3.6 & 25 & 8.9 & 0 & 0.0 & 280 & 100.0 \\
\hline Moruya & 285 & 88.8 & 6 & 1.9 & 29 & 9.0 & 1 & 0.3 & 321 & 100.0 \\
\hline Queanbeyan & 273 & 88.3 & 7 & 2.3 & 28 & 9.1 & 1 & 0.3 & 309 & 100.0 \\
\hline Bowral & 473 & 83.3 & 23 & 4.0 & 69 & 12.1 & 3 & 0.5 & 568 & 100.0 \\
\hline Broken Hill Base & 183 & 75.9 & 12 & 5.0 & 46 & 19.1 & 0 & 0.0 & 241 & 100.0 \\
\hline Other hospitals & 2030 & 79.9 & 65 & 2.6 & 432 & 17.0 & 14 & 0.6 & 2541 & 100.0 \\
\hline TOTAL & 5404 & 83.0 & 175 & 2.7 & 897 & 13.8 & 36 & 0.6 & 6512 & 100.0 \\
\hline \multicolumn{11}{|l|}{ Level 2} \\
\hline Wyong & 208 & 88.9 & 1 & 0.4 & 24 & 10.3 & 1 & 0.4 & 234 & 100.0 \\
\hline Other hospitals & 788 & 92.5 & 9 & 1.1 & 52 & 6.1 & 3 & 0.4 & 852 & 100.0 \\
\hline TOTAL & 996 & 91.7 & 10 & 0.9 & 76 & 7.0 & 4 & 0.4 & 1086 & 100.0 \\
\hline \multicolumn{11}{|l|}{ Private } \\
\hline Mater, North Sydney & 2186 & 86.3 & 246 & 9.7 & 99 & 3.9 & 1 & 0.0 & 2532 & 100.0 \\
\hline North Shore Private & 2259 & 84.9 & 238 & 8.9 & 155 & 5.8 & 9 & 0.3 & 2661 & 100.0 \\
\hline Sydney Adventist & 2005 & 88.2 & 0 & 0.0 & 262 & 11.5 & 6 & 0.3 & 2273 & 100.0 \\
\hline North Gosford Private & 753 & 89.0 & 30 & 3.5 & 63 & 7.4 & 0 & 0.0 & 846 & 100.0 \\
\hline Hurstville Private & 724 & 74.3 & 162 & 16.6 & 88 & 9.0 & 0 & 0.0 & 974 & 100.0 \\
\hline Kareena Private & 592 & 86.2 & 26 & 3.8 & 69 & 10.0 & 0 & 0.0 & 687 & 100.0 \\
\hline St. George Private & 1413 & 76.8 & 243 & 13.2 & 181 & 9.8 & 4 & 0.2 & 1841 & 100.0 \\
\hline Prince of Wales Private & 2028 & 88.8 & 146 & 6.4 & 110 & 4.8 & 1 & 0.0 & 2285 & 100.0 \\
\hline Norwest Private & 1013 & 82.1 & 65 & 5.3 & 154 & 12.5 & 2 & 0.2 & 1234 & 100.0 \\
\hline Sydney Southwest Private & 848 & 79.4 & 61 & 5.7 & 158 & 14.8 & 1 & 0.1 & 1068 & 100.0 \\
\hline Nepean Private & 698 & 74.4 & 105 & 11.2 & 133 & 14.2 & 2 & 0.2 & 938 & 100.0 \\
\hline Westmead Private & 1669 & 70.1 & 442 & 18.6 & 255 & 10.7 & 14 & 0.6 & 2380 & 100.0 \\
\hline Figtree Private & 816 & 79.5 & 29 & 2.8 & 181 & 17.6 & 0 & 0.0 & 1026 & 100.0 \\
\hline Newcastle Private & 1732 & 91.4 & 0 & 0.0 & 162 & 8.5 & 2 & 0.1 & 1896 & 100.0 \\
\hline Calvary, Wagga Wagga & 528 & 82.9 & 28 & 4.4 & 81 & 12.7 & 0 & 0.0 & 637 & 100.0 \\
\hline Other hospitals & 235 & 86.7 & 10 & 3.7 & 26 & 9.6 & 0 & 0.0 & 271 & 100.0 \\
\hline TOTAL & 19499 & 82.8 & 1831 & 7.8 & 2177 & 9.2 & 42 & 0.2 & 23549 & 100.0 \\
\hline TOTAL NSW* & 77155 & 80.5 & 6423 & 6.7 & 11559 & 12.1 & 700 & 0.7 & 95837 & 100.0 \\
\hline
\end{tabular}




\section{Baby discharge status}

Table 51 shows the discharge status of babies for Local Health Districts, and Table 52 for individual hospitals by maternity service level where at least 200 mothers gave birth in 2009

Table 51. Baby discharge status by Local Health District of hospital, NSW 2009

\begin{tabular}{|c|c|c|c|c|c|c|c|c|c|c|c|c|c|c|}
\hline \multirow[t]{3}{*}{ Local Health District } & \multicolumn{14}{|c|}{ Baby discharge status } \\
\hline & \multicolumn{2}{|c|}{ Discharged } & \multicolumn{2}{|c|}{ Transferred } & \multicolumn{2}{|c|}{ Stillborn } & \multicolumn{2}{|c|}{ Died } & \multicolumn{2}{|c|}{$\begin{array}{l}\text { Transferred } \\
\text { and died }\end{array}$} & \multicolumn{2}{|c|}{ Not stated } & \multicolumn{2}{|c|}{ Total } \\
\hline & No. & $\%$ & No. & $\%$ & No. & $\%$ & No. & $\%$ & No. & $\%$ & No. & $\%$ & No. & $\%$ \\
\hline Sydney & 6604 & 96.9 & 120 & 1.8 & 58 & 0.9 & 31 & 0.5 & 0 & 0.0 & 0 & 0.0 & 6813 & 100.0 \\
\hline South Western Sydney & 10116 & 96.3 & 279 & 2.7 & 77 & 0.7 & 37 & 0.4 & 0 & 0.0 & 0 & 0.0 & 10509 & 100.0 \\
\hline South Eastern Sydney & 7764 & 96.6 & 176 & 2.2 & 65 & 0.8 & 36 & 0.4 & 0 & 0.0 & 0 & 0.0 & 8041 & 100.0 \\
\hline Illawarra Shoalhaven & 3238 & 96.8 & 75 & 2.2 & 19 & 0.6 & 12 & 0.4 & 0 & 0.0 & 0 & 0.0 & 3344 & 100.0 \\
\hline Western Sydney & 8845 & 95.1 & 349 & 3.8 & 81 & 0.9 & 29 & 0.3 & 0 & 0.0 & 0 & 0.0 & 9304 & 100.0 \\
\hline Nepean Blue Mountains & 4710 & 95.0 & 200 & 4.0 & 31 & 0.6 & 16 & 0.3 & 0 & 0.0 & 0 & 0.0 & 4957 & 100.0 \\
\hline Northern Sydney & 4672 & 96.3 & 142 & 2.9 & 28 & 0.6 & 12 & 0.2 & 0 & 0.0 & 0 & 0.0 & 4854 & 100.0 \\
\hline Central Coast & 2740 & 96.9 & 66 & 2.3 & 15 & 0.5 & 7 & 0.2 & 0 & 0.0 & 0 & 0.0 & 2828 & 100.0 \\
\hline Hunter New England & 8176 & 92.0 & 600 & 6.8 & 70 & 0.8 & 40 & 0.5 & 0 & 0.0 & 0 & 0.0 & 8886 & 100.0 \\
\hline Northern NSW & 3553 & 94.6 & 174 & 4.6 & 22 & 0.6 & 8 & 0.2 & 0 & 0.0 & 0 & 0.0 & 3757 & 100.0 \\
\hline Mid North Coast & 2099 & 93.7 & 120 & 5.4 & 17 & 0.8 & 5 & 0.2 & 0 & 0.0 & 0 & 0.0 & 2241 & 100.0 \\
\hline Southern NSW & 1371 & 97.2 & 32 & 2.3 & 8 & 0.6 & 0 & 0.0 & 0 & 0.0 & 0 & 0.0 & 1411 & 100.0 \\
\hline Murrumbidgee & 1865 & 93.5 & 116 & 5.8 & 12 & 0.6 & 1 & 0.1 & 0 & 0.0 & 0 & 0.0 & 1994 & 100.0 \\
\hline Western NSW & 3023 & 87.3 & 423 & 12.2 & 13 & 0.4 & 3 & 0.1 & 0 & 0.0 & 0 & 0.0 & 3462 & 100.0 \\
\hline Far West & 237 & 97.9 & 4 & 1.7 & 1 & 0.4 & 0 & 0.0 & 0 & 0.0 & 0 & 0.0 & 242 & 100.0 \\
\hline Private Hospitals & 23348 & 98.8 & 189 & 0.8 & 81 & 0.3 & 10 & 0.0 & 2 & 0.0 & 0 & 0.0 & 23630 & 100.0 \\
\hline TOTAL* & 92521 & 95.9 & 3067 & 3.2 & 598 & 0.6 & 247 & 0.3 & 2 & 0.0 & 4 & 0.0 & 96439 & 100.0 \\
\hline
\end{tabular}

Source: NSW Perinatal Data Collection (HOIST). Centre for Epidemiology and Research, NSW Ministry of Health.

*Total includes births at home assisted by independent midwives.

Table 52. Baby discharge status by hospital and maternity service level, NSW 2009\#

\begin{tabular}{|c|c|c|c|c|c|c|c|c|c|c|c|c|c|c|}
\hline \multirow[t]{3}{*}{ Maternity service level-hospital } & \multicolumn{14}{|c|}{ Baby discharge status } \\
\hline & \multicolumn{2}{|c|}{ Discharged } & \multicolumn{2}{|c|}{ Transferred } & \multicolumn{2}{|c|}{ Stillborn } & \multicolumn{2}{|c|}{ Died } & \multicolumn{2}{|c|}{$\begin{array}{l}\text { Transferred } \\
\text { and died }\end{array}$} & \multicolumn{2}{|c|}{ Not stated } & \multicolumn{2}{|c|}{ TOTAL } \\
\hline & No. & $\%$ & No. & $\%$ & No. & $\%$ & No. & $\%$ & No. & $\%$ & No. & $\%$ & No. & $\%$ \\
\hline \multicolumn{15}{|l|}{ Level 6} \\
\hline Royal Prince Alfred & 4931 & 96.5 & 104 & 2.0 & 46 & 0.9 & 30 & 0.6 & 0 & 0 & 0 & 0 & 5111 & 100.0 \\
\hline Royal North Shore & 1972 & 93.6 & 104 & 4.9 & 21 & 1.0 & 9 & 0.4 & 0 & 0 & 0 & 0 & 2106 & 100.0 \\
\hline Royal Hospital for Women & 4020 & 95.6 & 112 & 2.7 & 49 & 1.2 & 22 & 0.5 & 0 & 0 & 0 & 0 & 4203 & 100.0 \\
\hline Liverpool & 2888 & 91.9 & 203 & 6.5 & 27 & 0.9 & 23 & 0.7 & 0 & 0 & 0 & 0 & 3141 & 100.0 \\
\hline Nepean & 3445 & 94.3 & 163 & 4.5 & 30 & 0.8 & 15 & 0.4 & 0 & 0 & 0 & 0 & 3653 & 100.0 \\
\hline Westmead & 4370 & 92.5 & 274 & 5.8 & 59 & 1.2 & 21 & 0.4 & 0 & 0 & 0 & 0 & 4724 & 100.0 \\
\hline John Hunter & 3535 & 93.2 & 184 & 4.9 & 36 & 0.9 & 37 & 1.0 & 0 & 0 & 0 & 0 & 3792 & 100.0 \\
\hline TOTAL & 25161 & 94.1 & 1144 & 4.3 & 268 & 1.0 & 157 & 0.6 & 0 & 0 & 0 & 0 & 26730 & 100.0 \\
\hline \multicolumn{15}{|l|}{ Level 5} \\
\hline Gosford & 2526 & 97.4 & 46 & 1.8 & 15 & 0.6 & 7 & 0.3 & 0 & 0 & 0 & 0 & 2594 & 100.0 \\
\hline St. George & 2485 & 97.2 & 48 & 1.9 & 12 & 0.5 & 11 & 0.4 & 0 & 0 & 0 & 0 & 2556 & 100.0 \\
\hline Blacktown & 3161 & 97.7 & 55 & 1.7 & 14 & 0.4 & 6 & 0.2 & 0 & 0 & 0 & 0 & 3236 & 100.0 \\
\hline Lismore Base & 1307 & 97.5 & 23 & 1.7 & 7 & 0.5 & 4 & 0.3 & 0 & 0 & 0 & 0 & 1341 & 100.0 \\
\hline Tweed Heads & 1365 & 90.1 & 133 & 8.8 & 13 & 0.9 & 4 & 0.3 & 0 & 0 & 0 & 0 & 1515 & 100.0 \\
\hline Port Macquarie Base & 741 & 93.0 & 45 & 5.6 & 7 & 0.9 & 4 & 0.5 & 0 & 0 & 0 & 0 & 797 & 100.0 \\
\hline Wollongong & 2292 & 96.9 & 57 & 2.4 & 12 & 0.5 & 5 & 0.2 & 0 & 0 & 0 & 0 & 2366 & 100.0 \\
\hline Maitland & 1341 & 86.7 & 188 & 12.2 & 16 & 1.0 & 1 & 0.1 & 0 & 0 & 0 & 0 & 1546 & 100.0 \\
\hline Wagga Wagga Base & 677 & 88.5 & 78 & 10.2 & 9 & 1.2 & 1 & 0.1 & 0 & 0 & 0 & 0 & 765 & 100.0 \\
\hline TOTAL & 15895 & 95.1 & 673 & 4.0 & 105 & 0.6 & 43 & 0.3 & 0 & 0 & 0 & 0 & 16716 & 100.0 \\
\hline \multicolumn{15}{|l|}{ Level 4} \\
\hline Canterbury & 1673 & 98.3 & 16 & 0.9 & 12 & 0.7 & 1 & 0.1 & 0 & 0 & 0 & 0 & 1702 & 100.0 \\
\hline Hornsby & 1146 & 98.2 & 15 & 1.3 & 3 & 0.3 & 3 & 0.3 & 0 & 0 & 0 & 0 & 1167 & 100.0 \\
\hline Manly & 1127 & 98.5 & 14 & 1.2 & 3 & 0.3 & 0 & 0 & 0 & 0 & 0 & 0 & 1144 & 100.0 \\
\hline
\end{tabular}


Table 52. (Continued)

\begin{tabular}{|c|c|c|c|c|c|c|c|c|c|c|c|c|c|c|}
\hline \multirow[t]{3}{*}{ Maternity service level-hospital } & \multicolumn{14}{|c|}{ Baby discharge status } \\
\hline & \multicolumn{2}{|c|}{ Discharged } & \multicolumn{2}{|c|}{ Transferred } & \multicolumn{2}{|c|}{ Stillborn } & \multicolumn{2}{|c|}{ Died } & \multicolumn{2}{|c|}{$\begin{array}{l}\text { Transferred } \\
\text { and died }\end{array}$} & \multicolumn{2}{|c|}{ Not stated } & \multicolumn{2}{|c|}{ TOTAL } \\
\hline & No. & $\%$ & No. & $\%$ & No. & $\%$ & No. & $\%$ & No. & $\%$ & No. & $\%$ & No. & $\%$ \\
\hline Mona Vale & 311 & 98.4 & 4 & 1.3 & 1 & 0.3 & 0 & 0 & 0 & 0 & 0 & 0 & 316 & 100.0 \\
\hline Sutherland & 1259 & 98.2 & 16 & 1.2 & 4 & 0.3 & 3 & 0.2 & 0 & 0 & 0 & 0 & 1282 & 100.0 \\
\hline Auburn & 1314 & 97.8 & 20 & 1.5 & 8 & 0.6 & 2 & 0.1 & 0 & 0 & 0 & 0 & 1344 & 100.0 \\
\hline Fairfield & 1861 & 98.3 & 18 & 1.0 & 13 & 0.7 & 2 & 0.1 & 0 & 0 & 0 & 0 & 1894 & 100.0 \\
\hline Campbelltown & 2655 & 98.0 & 30 & 1.1 & 19 & 0.7 & 6 & 0.2 & 0 & 0 & 0 & 0 & 2710 & 100.0 \\
\hline Bankstown-Lidcombe & 2149 & 97.9 & 22 & 1.0 & 17 & 0.8 & 6 & 0.3 & 0 & 0 & 0 & 0 & 2194 & 100.0 \\
\hline Hawkesbury & 857 & 98.7 & 9 & 1.0 & 1 & 0.1 & 1 & 0.1 & 0 & 0 & 0 & 0 & 868 & 100.0 \\
\hline Coffs Harbour & 1021 & 94.0 & 58 & 5.3 & 6 & 0.6 & 1 & 0.1 & 0 & 0 & 0 & 0 & 1086 & 100.0 \\
\hline Grafton Base & 486 & 98.4 & 6 & 1.2 & 2 & 0.4 & 0 & 0 & 0 & 0 & 0 & 0 & 494 & 100.0 \\
\hline Tamworth Base & 701 & 83.1 & 138 & 16.4 & 5 & 0.6 & 0 & 0 & 0 & 0 & 0 & 0 & 844 & 100.0 \\
\hline Manning Base & 739 & 97.2 & 14 & 1.8 & 7 & 0.9 & 0 & 0 & 0 & 0 & 0 & 0 & 760 & 100.0 \\
\hline Dubbo Base & 980 & 80.3 & 236 & 19.3 & 4 & 0.3 & 1 & 0.1 & 0 & 0 & 0 & 0 & 1221 & 100.0 \\
\hline Orange Base & 825 & 91.4 & 72 & 8.0 & 6 & 0.7 & 0 & 0 & 0 & 0 & 0 & 0 & 903 & 100.0 \\
\hline Goulburn Base & 290 & 96.0 & 10 & 3.3 & 2 & 0.7 & 0 & 0 & 0 & 0 & 0 & 0 & 302 & 100.0 \\
\hline Shoalhaven & 860 & 97.0 & 14 & 1.6 & 7 & 0.8 & 6 & 0.7 & 0 & 0 & 0 & 0 & 887 & 100.0 \\
\hline Griffith Base & 403 & 97.1 & 10 & 2.4 & 2 & 0.5 & 0 & 0 & 0 & 0 & 0 & 0 & 415 & 100.0 \\
\hline TOTAL & 20657 & 95.9 & 722 & 3.4 & 122 & 0.6 & 32 & 0.1 & 0 & 0 & 0 & 0 & 21533 & 100.0 \\
\hline \multicolumn{15}{|l|}{ Level 3} \\
\hline Blue Mountains & 236 & 95.9 & 10 & 4.1 & 0 & 0 & 0 & 0 & 0 & 0 & 0 & 0 & 246 & 100.0 \\
\hline Kempsey & 291 & 94.8 & 13 & 4.2 & 3 & 1.0 & 0 & 0 & 0 & 0 & 0 & 0 & 307 & 100.0 \\
\hline Murwillumbah & 205 & 98.1 & 4 & 1.9 & 0 & 0 & 0 & 0 & 0 & 0 & 0 & 0 & 209 & 100.0 \\
\hline Armidale & 428 & 95.7 & 17 & 3.8 & 1 & 0.2 & 1 & 0.2 & 0 & 0 & 0 & 0 & 447 & 100.0 \\
\hline Inverell & 247 & 96.1 & 7 & 2.7 & 2 & 0.8 & 1 & 0.4 & 0 & 0 & 0 & 0 & 257 & 100.0 \\
\hline Mudgee & 223 & 97.4 & 4 & 1.7 & 2 & 0.9 & 0 & 0 & 0 & 0 & 0 & 0 & 229 & 100.0 \\
\hline Bathurst Base & 495 & 87.5 & 68 & 12.0 & 1 & 0.2 & 2 & 0.4 & 0 & 0 & 0 & 0 & 566 & 100.0 \\
\hline Bega & 276 & 98.2 & 4 & 1.4 & 1 & 0.4 & 0 & 0 & 0 & 0 & 0 & 0 & 281 & 100.0 \\
\hline Moruya & 309 & 95.1 & 12 & 3.7 & 4 & 1.2 & 0 & 0 & 0 & 0 & 0 & 0 & 325 & 100.0 \\
\hline Queanbeyan & 305 & 98.7 & 4 & 1.3 & 0 & 0 & 0 & 0 & 0 & 0 & 0 & 0 & 309 & 100.0 \\
\hline Bowral & 562 & 98.8 & 6 & 1.1 & 1 & 0.2 & 0 & 0 & 0 & 0 & 0 & 0 & 569 & 100.0 \\
\hline Broken Hill Base & 237 & 97.9 & 4 & 1.7 & 1 & 0.4 & 0 & 0 & 0 & 0 & 0 & 0 & 242 & 100.0 \\
\hline Other hospitals & 2431 & 95.5 & 110 & 4.3 & 4 & 0.2 & 0 & 0 & 0 & 0 & 0 & 0 & 2545 & 100.0 \\
\hline TOTAL & 6245 & 95.6 & 263 & 4.0 & 20 & 0.3 & 4 & 0.1 & 0 & 0 & 0 & 0 & 6532 & 100.0 \\
\hline \multicolumn{15}{|l|}{ Level 2} \\
\hline Wyong & 214 & 91.5 & 20 & 8.5 & 0 & 0 & 0 & 0 & 0 & 0 & 0 & 0 & 234 & 100.0 \\
\hline Other hospitals & 819 & 95.9 & 32 & 3.7 & 2 & 0.2 & 1 & 0.1 & 0 & 0 & 0 & 0 & 854 & 100.0 \\
\hline TOTAL & 1033 & 94.9 & 52 & 4.8 & 2 & 0.2 & 1 & 0.1 & 0 & 0 & 0 & 0 & 1088 & 100.0 \\
\hline \multicolumn{15}{|l|}{ Private } \\
\hline Mater, North Sydney & 2518 & 99.3 & 14 & 0.6 & 4 & 0.2 & 0 & 0 & 0 & 0 & 0 & 0 & 2536 & 100.0 \\
\hline North Shore Private & 2638 & 98.8 & 18 & 0.7 & 10 & 0.4 & 5 & 0.2 & 0 & 0 & 0 & 0 & 2671 & 100.0 \\
\hline Sydney Adventist & 2268 & 99.2 & 4 & 0.2 & 13 & 0.6 & 1 & 0.0 & 0 & 0 & 0 & 0 & 2286 & 100.0 \\
\hline North Gosford Private & 840 & 99.1 & 6 & 0.7 & 2 & 0.2 & 0 & 0 & 0 & 0 & 0 & 0 & 848 & 100.0 \\
\hline Hurstville Private & 957 & 98.2 & 17 & 1.7 & 1 & 0.1 & 0 & 0 & 0 & 0 & 0 & 0 & 975 & 100.0 \\
\hline Kareena Private & 683 & 99.1 & 4 & 0.6 & 2 & 0.3 & 0 & 0 & 0 & 0 & 0 & 0 & 689 & 100.0 \\
\hline St. George Private & 1827 & 99.0 & 13 & 0.7 & 4 & 0.2 & 1 & 0.1 & 0 & 0 & 0 & 0 & 1845 & 100.0 \\
\hline Prince of Wales Private & 2277 & 99.4 & 8 & 0.3 & 6 & 0.3 & 0 & 0 & 0 & 0 & 0 & 0 & 2291 & 100.0 \\
\hline Norwest Private & 1218 & 98.1 & 14 & 1.1 & 7 & 0.6 & 2 & 0.2 & 0 & 0 & 0 & 0 & 1241 & 100.0 \\
\hline Sydney Southwest Private & 1062 & 99.3 & 6 & 0.6 & 1 & 0.1 & 0 & 0 & 0 & 0 & 0 & 0 & 1069 & 100.0 \\
\hline Nepean Private & 921 & 98.0 & 16 & 1.7 & 2 & 0.2 & 0 & 0 & 1 & 0.1 & 0 & 0 & 940 & 100.0 \\
\hline Westmead Private & 2355 & 98.4 & 25 & 1.0 & 13 & 0.5 & 0 & 0 & 0 & 0 & 0 & 0 & 2393 & 100.0 \\
\hline Figtree Private & 1017 & 98.5 & 9 & 0.9 & 7 & 0.7 & 0 & 0 & 0 & 0 & 0 & 0 & 1033 & 100.0 \\
\hline Newcastle Private & 1871 & 98.3 & 23 & 1.2 & 7 & 0.4 & 1 & 0.1 & 1 & 0.1 & 0 & 0 & 1903 & 100.0 \\
\hline Calvary, Wagga Wagga & 632 & 98.9 & 5 & 0.8 & 2 & 0.3 & 0 & 0 & 0 & 0 & 0 & 0 & 639 & 100.0 \\
\hline Other hospitals & 264 & 97.4 & 7 & 2.6 & 0 & 0 & 0 & 0 & 0 & 0 & 0 & 0 & 271 & 100.0 \\
\hline TOTAL & 23348 & 98.8 & 189 & 0.8 & 81 & 0.3 & 10 & 0.0 & 2 & 0.0 & 0 & 0 & 23630 & 100.0 \\
\hline TOTAL NSW* & 92521 & 95.9 & 3067 & 3.2 & 598 & 0.6 & 247 & 0.3 & 2 & 0.0 & 4 & 0.0 & 96439 & 100.0 \\
\hline
\end{tabular}

Source: NSW Perinatal Data Collection (HOIST). Centre for Epidemiology and Research, NSW Ministry of Health

\#Hospitals with more than 200 births are identified individually. Maternity service levels are described on page 13 of the Methods chapter.

*Total NSW includes births at home assisted by independent midwives and births at level 1 hospitals. 


\section{Postnatal length of stay}

Table 53 shows the mother's postnatal length of stay in the hospital of birth for hospitals where at least 200 mothers gave birth in 2009.

\section{Table 53. Average maternal postnatal length of stay in hospital of birth, NSW 2007-2009\#}

\begin{tabular}{|c|c|c|c|c|c|c|c|}
\hline \multirow[t]{2}{*}{$\begin{array}{l}\text { Local Health District- } \\
\text { hospital }\end{array}$} & \multicolumn{3}{|c|}{$\begin{array}{c}\text { Average postnatal length of stay } \\
\text { (days) }\end{array}$} & \multirow[t]{2}{*}{$\begin{array}{l}\text { Local Health District- } \\
\text { hospital }\end{array}$} & \multicolumn{3}{|c|}{$\begin{array}{c}\text { Average postnatal length of stay } \\
\text { (days) }\end{array}$} \\
\hline & 2007 & 2008 & 2009 & & 2007 & 2008 & 2009 \\
\hline Sydney & & & & Northern NSW & & & \\
\hline Canterbury & 3.1 & 2.9 & 2.8 & Grafton Base & 3.7 & 3.5 & 3.6 \\
\hline Royal Prince Alfred & 3.6 & 3.5 & 3.4 & Lismore Base & 3.3 & 3.1 & 3.2 \\
\hline TOTAL & 3.4 & 3.4 & 3.2 & Murwillumbah & 3.5 & 3.2 & 3.2 \\
\hline South Western Sydney & & & & Tweed Heads & 2.7 & 2.7 & 2.7 \\
\hline Fairfield & 2.6 & 2.5 & 2.5 & Other hospitals & 3.0 & 1.0 & 0.0 \\
\hline Liverpool & 3.0 & 3.0 & 2.9 & TOTAL & 3.1 & 3.0 & 3.0 \\
\hline Campbelltown & 2.7 & 2.7 & 2.5 & Mid North Coast & & & \\
\hline Bankstown-Lidcombe & 2.9 & 2.6 & 2.5 & Coffs Harbour & 3.7 & 3.8 & 3.7 \\
\hline Bowral & 2.6 & 2.8 & 2.8 & Kempsey & 3.1 & 2.9 & 2.8 \\
\hline Other hospitals & 0.0 & 7.0 & 0.0 & Port Macquarie Base & 3.4 & 3.2 & 3.3 \\
\hline TOTAL & 2.8 & 2.7 & 2.7 & Other hospitals & 4.4 & 2.3 & 2.4 \\
\hline South Eastern Sydney & & & & TOTAL & 3.5 & 3.4 & 3.4 \\
\hline St. George & 3.5 & 3.1 & 2.7 & Southern NSW & & & \\
\hline Sutherland & 2.9 & 2.8 & 2.7 & Bega & 2.4 & 2.3 & 2.1 \\
\hline Royal Hospital for Women & 3.5 & 3.9 & 3.7 & Goulburn Base & 3.3 & 3.3 & 3.3 \\
\hline TOTAL & 3.4 & 3.5 & 3.2 & Moruya & 2.6 & 2.6 & 2.7 \\
\hline Illawarra Shoalhaven & & & & Queanbeyan & 3.3 & 3.3 & 3.7 \\
\hline Shoalhaven & 2.8 & 2.6 & 2.5 & Other hospitals & 0.0 & 0.0 & 0.0 \\
\hline Wollongong & 2.8 & 2.8 & 2.7 & TOTAL & 3.1 & 3.0 & 3.0 \\
\hline Other hospitals & 2.0 & 0.0 & 0.0 & Murrumbidgee & & & \\
\hline TOTAL & 2.8 & 2.8 & 2.6 & Griffith Base & 3.3 & 3.1 & 2.9 \\
\hline Western Sydney & & & & Wagga Wagga Base & 2.8 & 2.8 & 2.4 \\
\hline Auburn & 2.6 & 2.0 & 2.0 & Other hospitals & 1.7 & 0.0 & 0.0 \\
\hline Blacktown & 2.9 & 2.8 & 2.7 & TOTAL & 3.3 & 3.1 & 2.9 \\
\hline Westmead & 2.7 & 2.6 & 2.4 & Western NSW & & & \\
\hline TOTAL & 2.8 & 2.6 & 2.4 & Dubbo Base & 2.9 & 2.8 & 2.6 \\
\hline Nepean Blue Mountains & & & & Mudgee & 2.9 & 3.1 & 2.5 \\
\hline Blue Mountains & 3.3 & 3.4 & 3.4 & Bathurst Base & 3.2 & 2.9 & 2.7 \\
\hline Nepean & 2.9 & 2.6 & 2.4 & Orange Base & 3.3 & 3.4 & 2.9 \\
\hline Hawkesbury & 3.2 & 3.2 & 3.2 & Other hospitals & 3.7 & 2.0 & 0.9 \\
\hline Lithgow & 3.0 & 2.9 & 3.2 & TOTAL & 3.2 & 3.0 & 2.7 \\
\hline TOTAL & 2.9 & 2.8 & 2.6 & Far West & & & \\
\hline Northern Sydney & & & & Broken Hill Base & 3.5 & 3.3 & 3.5 \\
\hline Hornsby & 2.5 & 2.5 & 2.4 & TOTAL & 3.5 & 3.3 & 3.5 \\
\hline Manly & 2.9 & 3.1 & 2.7 & Private Hospitals & & & \\
\hline Mona Vale & 2.6 & 2.7 & 2.5 & Mater, North Sydney & 4.6 & 4.5 & 4.5 \\
\hline Royal North Shore & 3.1 & 3.0 & 2.8 & North Shore Private & 4.4 & 4.5 & 4.4 \\
\hline Ryde & 0.5 & 0.9 & 0.4 & Sydney Adventist & 4.7 & 4.7 & 4.6 \\
\hline TOTAL & 2.8 & 2.8 & 2.6 & North Gosford Private & 4.6 & 4.8 & 4.6 \\
\hline Central Coast & & & & Hurstville Private & 4.7 & 4.5 & 4.5 \\
\hline Gosford & 2.2 & 2.2 & 2.2 & Kareena Private & 4.5 & 4.6 & 4.5 \\
\hline Wyong & 1.8 & 1.3 & 1.1 & St. George Private & 4.4 & 4.3 & 4.3 \\
\hline TOTAL & 2.2 & 2.2 & 2.1 & Prince of Wales Private & 4.8 & 4.4 & 4.5 \\
\hline Hunter New England & & & & Norwest Private & 4.7 & 4.3 & 4.3 \\
\hline Armidale & 3.4 & 3.3 & 3.1 & Sydney Southwest Private & 4.5 & 4.4 & 4.3 \\
\hline Inverell & 3.5 & 3.0 & 3.1 & Nepean Private & 5.2 & 4.8 & 4.8 \\
\hline Tamworth Base & 3.2 & 3.1 & 3.0 & Westmead Private & 4.0 & 3.9 & 3.9 \\
\hline Manning Base & 3.3 & 3.1 & 3.1 & Figtree Private & 5.3 & 5.3 & 5.3 \\
\hline Maitland & 2.5 & 2.4 & 2.5 & Newcastle Private & 4.1 & 4.1 & 3.9 \\
\hline John Hunter & 3.1 & 3.0 & 2.8 & Calvary, Wagga Wagga & 5.0 & 5.0 & 4.5 \\
\hline Other hospitals & 2.1 & 3.2 & 2.2 & Other hospitals & 4.9 & 4.5 & 4.5 \\
\hline \multirow[t]{2}{*}{ TOTAL } & 3.0 & 2.8 & 2.8 & TOTAL & 4.5 & 4.5 & 4.4 \\
\hline & & & & TOTAL NSW & 3.4 & 3.3 & 3.2 \\
\hline
\end{tabular}




\section{Clinical Indicators}

Selected clinical indicators for obstetrics are reported in this section. The definitions of the clinical indicators follow those described in Version 6 of the Australian Council on Healthcare Standards Clinical Indicator User's Manual 2008.1

\section{Reference}

1. Australian Council on Healthcare Standards. Obstetrics Indicators-Clinical Indicator Users' Manual, version 6. Sydney: ACHS, 2008.

\section{Outcome of selected primipara. Indicator 1.1}

Definition: The number of selected primipara who have a spontaneous vaginal birth as a percentage of the total number of selected primipara who give birth.

A selected primipara is defined as a woman who is 20-34 years of age at the time of giving birth; giving birth for the first time at greater than 20 weeks gestation; singleton pregnancy; cephalic presentation; and at $37^{\circ}$ to $41^{\circ}$ weeks gestation.

Table 54 shows aggregate information for hospitals where at least 200 mothers gave birth in 2009, totals for hospitals within each Local Health District, and the NSW total.

Table 54. 1.1 Selected primipara who have a spontaneous vaginal birth by Local Health District and hospital, NSW 2007-2009\#

\begin{tabular}{|c|c|c|c|c|c|c|c|}
\hline Local Health District-hospital & $\begin{array}{c}2007 \\
\%\end{array}$ & $\begin{array}{c}2008 \\
\%\end{array}$ & $\begin{array}{c}2009 \\
\%\end{array}$ & Local Health District-hospital & $\begin{array}{c}2007 \\
\%\end{array}$ & $\begin{array}{c}2008 \\
\%\end{array}$ & $\begin{array}{c}2009 \\
\%\end{array}$ \\
\hline Sydney & & & & Northern NSW & & & \\
\hline Canterbury & 58.0 & 57.2 & 55.2 & Grafton Base & 57.5 & 55.9 & 57.6 \\
\hline Royal Prince Alfred & 53.8 & 54.0 & 51.8 & Lismore Base & 60.3 & 60.0 & 59.2 \\
\hline TOTAL & 54.7 & 54.6 & 52.6 & Murwillumbah & 61.7 & 63.7 & 87.5 \\
\hline South Western Sydney & & & & Tweed Heads & 65.2 & 66.8 & 67.8 \\
\hline Fairfield & 73.5 & 75.8 & 71.2 & Other hospitals & 84.7 & 98.0 & 93.3 \\
\hline Liverpool & 62.6 & 65.0 & 67.6 & TOTAL & 63.5 & 64.6 & 65.8 \\
\hline Campbelltown & 67.6 & 66.9 & 64.8 & Mid North Coast & & & \\
\hline Bankstown-Lidcombe & 62.1 & 63.6 & 62.2 & Coffs Harbour & 60.2 & 56.1 & 51.1 \\
\hline Bowral & 48.9 & 55.6 & 52.9 & Kempsey & 73.5 & 73.8 & 71.2 \\
\hline Other hospitals & 0.0 & 0.0 & 100.0 & Port Macquarie Base & 62.4 & 57.7 & 58.4 \\
\hline TOTAL & 65.0 & 66.7 & 65.7 & Other hospitals & 100.0 & 100.0 & 75.0 \\
\hline South Eastern Sydney & & & & TOTAL & 63.3 & 59.2 & 56.0 \\
\hline St. George & 57.6 & 50.9 & 55.2 & Southern NSW & & & \\
\hline Sutherland & 66.2 & 70.3 & 57.4 & Bega & 61.5 & 56.3 & 68.4 \\
\hline Royal Hospital for Women & 49.5 & 49.4 & 51.4 & Goulburn Base & 52.0 & 55.6 & 38.0 \\
\hline TOTAL & 54.6 & 53.1 & 53.7 & Moruya & 58.2 & 53.5 & 54.5 \\
\hline Illawarra Shoalhaven & & & & Queanbeyan & 68.0 & 63.6 & 60.0 \\
\hline Shoalhaven & 57.2 & 55.8 & 57.1 & Other hospitals & 67.1 & 69.3 & 75.6 \\
\hline Wollongong & 55.9 & 59.8 & 59.0 & TOTAL & 61.4 & 60.1 & 57.6 \\
\hline Other hospitals & 58.1 & 60.0 & 41.7 & Murrumbidgee & & & \\
\hline TOTAL & 56.2 & 58.9 & 58.0 & Griffith Base & 64.2 & 58.6 & 59.4 \\
\hline Western Sydney & & & & Wagga Wagga Base & 54.3 & 49.1 & 51.1 \\
\hline Auburn & 69.2 & 70.8 & 63.7 & Other hospitals & 49.5 & 52.7 & 51.5 \\
\hline Blacktown & 54.8 & 54.4 & 50.7 & TOTAL & 54.8 & 52.7 & 53.2 \\
\hline Westmead & 57.2 & 50.9 & 50.6 & Western NSW & & & \\
\hline TOTAL & 58.4 & 54.8 & 52.4 & Dubbo Base & 54.8 & 57.1 & 62.2 \\
\hline Nepean Blue Mountains & & & & Mudgee & 56.7 & 57.9 & 64.3 \\
\hline Blue Mountains & 55.7 & 47.2 & 51.1 & Bathurst Base & 64.9 & 66.7 & 59.4 \\
\hline Nepean & 49.0 & 47.5 & 49.0 & Orange Base & 50.6 & 56.4 & 46.0 \\
\hline Hawkesbury & 50.0 & 45.9 & 44.8 & Other hospitals & 56.5 & 64.8 & 60.0 \\
\hline Other hospitals & 63.0 & 59.6 & 64.5 & TOTAL & 55.7 & 59.8 & 57.6 \\
\hline TOTAL & 50.2 & 47.8 & 48.6 & Far West & & & \\
\hline Northern Sydney & & & & Broken Hill Base & 53.8 & 47.9 & 52.9 \\
\hline Hornsby & 52.3 & 49.0 & 53.6 & TOTAL & 53.8 & 47.9 & 52.9 \\
\hline Manly & 63.4 & 55.0 & 49.6 & Private Hospitals & & & \\
\hline Mona Vale & 44.2 & 45.8 & 48.3 & Mater, North Sydney & 29.8 & 28.2 & 30.1 \\
\hline Royal North Shore & 54.5 & 54.8 & 46.7 & North Shore Private & 33.9 & 31.3 & 36.1 \\
\hline Other hospitals & 100.0 & 100.0 & 100.0 & Sydney Adventist & 44.0 & 42.8 & 47.2 \\
\hline TOTAL & 55.0 & 53.6 & 50.2 & North Gosford Private & 34.1 & 30.7 & 29.8 \\
\hline Central Coast & & & & Hurstville Private & 35.5 & 36.7 & 39.9 \\
\hline Gosford & 43.7 & 46.4 & 43.6 & Kareena Private & 21.9 & 20.9 & 35.3 \\
\hline Wyong & 83.8 & 95.8 & 100.0 & St. George Private & 35.1 & 31.3 & 25.6 \\
\hline TOTAL & 47.6 & 48.2 & 46.9 & Prince of Wales Private & 39.7 & 40.8 & 35.5 \\
\hline Hunter New England & & & & Norwest Private & 41.9 & 47.5 & 40.1 \\
\hline Armidale & 71.6 & 59.5 & 73.5 & Sydney Southwest Private & 55.2 & 51.8 & 48.9 \\
\hline Inverell & 43.1 & 39.2 & 42.3 & Nepean Private & 44.1 & 42.1 & 48.0 \\
\hline Tamworth Base & 46.4 & 45.3 & 53.7 & Westmead Private & 43.5 & 40.6 & 39.4 \\
\hline Manning Base & 59.7 & 63.0 & 57.3 & Figtree Private & 41.7 & 45.8 & 38.3 \\
\hline Maitland & 58.0 & 56.0 & 50.2 & Newcastle Private & 41.8 & 41.7 & 45.8 \\
\hline John Hunter & 60.4 & 59.9 & 59.5 & Calvary, Wagga Wagga & 36.8 & 37.1 & 39.2 \\
\hline Other hospitals & 69.5 & 68.2 & 71.3 & Other hospitals & 31.9 & 43.5 & 34.7 \\
\hline \multirow[t]{2}{*}{ TOTAL } & 59.8 & 58.9 & 58.6 & TOTAL & 38.9 & 38.0 & 38.1 \\
\hline & & & & TOTAL NSW* & 52.7 & 52.1 & 51.4 \\
\hline
\end{tabular}


Outcome of selected primipara.

\section{Indicator 1.2}

Definition: The number of selected primipara who undergo induction of labour as a percentage of the total number of selected primipara who give birth.

A selected primipara is defined as a woman who is 20-34 years of age at the time of giving birth; giving birth for the first time at greater than 20 weeks gestation; singleton pregnancy; cephalic presentation; and at $37^{\circ}$ to $41^{\circ}$ weeks gestation.

Table 55 shows aggregate information for hospitals where at least 200 mothers gave birth in 2009, totals for hospitals within each Local Health District, and the NSW total.

Table 55. 1.2 Selected primipara who undergo induction of labour by Local Health District and hospital, NSW 2007-2009\#

\begin{tabular}{|c|c|c|c|c|c|c|c|}
\hline Local Health District-hospital & $\begin{array}{c}2007 \\
\%\end{array}$ & $\begin{array}{c}2008 \\
\%\end{array}$ & $\begin{array}{c}2009 \\
\%\end{array}$ & Local Health District-hospital & $\begin{array}{c}2007 \\
\%\end{array}$ & $\begin{array}{c}2008 \\
\%\end{array}$ & $\begin{array}{c}2009 \\
\%\end{array}$ \\
\hline Sydney & & & & Northern NSW & & & \\
\hline Canterbury & 28.5 & 27.8 & 28.2 & Grafton Base & 32.5 & 40.5 & 31.4 \\
\hline Royal Prince Alfred & 25.7 & 25.1 & 27.6 & Lismore Base & 33.9 & 24.0 & 25.6 \\
\hline TOTAL & 26.3 & 25.6 & 27.8 & Murwillumbah & 20.2 & 21.6 & 10.0 \\
\hline South Western Sydney & & & & Tweed Heads & 27.8 & 26.6 & 30.6 \\
\hline Fairfield & 23.7 & 19.0 & 23.1 & Other hospitals & 0.0 & 0.0 & 0.0 \\
\hline Liverpool & 27.3 & 31.2 & 28.9 & TOTAL & 28.0 & 25.4 & 26.8 \\
\hline Campbelltown & 30.6 & 27.3 & 32.2 & Mid North Coast & & & \\
\hline Bankstown-Lidcombe & 33.5 & 28.3 & 33.8 & Coffs Harbour & 20.8 & 23.5 & 23.8 \\
\hline Bowral & 35.1 & 36.8 & 21.4 & Kempsey & 8.2 & 31.1 & 23.7 \\
\hline Other hospitals & 0.0 & 0.0 & 0.0 & Port Macquarie Base & 32.5 & 24.5 & 28.1 \\
\hline TOTAL & 29.1 & 27.5 & 29.4 & Other hospitals & 0.0 & 0.0 & 0.0 \\
\hline South Eastern Sydney & & & & TOTAL & 23.9 & 24.4 & 25.1 \\
\hline St. George & 35.1 & 39.7 & 35.9 & Southern NSW & & & \\
\hline Sutherland & 30.5 & 29.9 & 37.7 & Bega & 33.3 & 27.1 & 29.8 \\
\hline Royal Hospital for Women & 35.2 & 33.8 & 32.8 & Goulburn Base & 8.0 & 9.7 & 15.5 \\
\hline TOTAL & 34.5 & 35.1 & 34.7 & Moruya & 27.8 & 29.6 & 24.7 \\
\hline Illawarra Shoalhaven & & & & Queanbeyan & 26.7 & 29.9 & 15.6 \\
\hline Shoalhaven & 28.9 & 32.0 & 30.7 & Other hospitals & 22.0 & 18.7 & 13.3 \\
\hline Wollongong & 39.7 & 43.2 & 43.7 & TOTAL & 22.6 & 22.7 & 19.7 \\
\hline Other hospitals & 54.8 & 30.0 & 37.5 & Murrumbidgee & & & \\
\hline TOTAL & 38.1 & 40.5 & 40.5 & Griffith Base & 19.5 & 15.2 & 24.8 \\
\hline Western Sydney & & & & Wagga Wagga Base & 31.4 & 26.0 & 35.1 \\
\hline Auburn & 32.3 & 29.5 & 30.3 & Other hospitals & 32.4 & 27.3 & 30.7 \\
\hline Blacktown & 43.2 & 40.4 & 43.9 & TOTAL & 28.9 & 24.0 & 31.1 \\
\hline Westmead & 43.0 & 44.5 & 42.7 & Western NSW & & & \\
\hline TOTAL & 41.3 & 41.1 & 41.4 & Dubbo Base & 31.2 & 34.5 & 29.7 \\
\hline Nepean Blue Mountains & & & & Mudgee & 28.3 & 31.6 & 37.5 \\
\hline Blue Mountains & 35.4 & 26.4 & 51.1 & Bathurst Base & 23.9 & 16.7 & 16.9 \\
\hline Nepean & 37.9 & 41.3 & 40.7 & Orange Base & 35.9 & 33.6 & 38.6 \\
\hline Hawkesbury & 43.0 & 39.7 & 42.3 & Other hospitals & 35.5 & 27.0 & 26.0 \\
\hline Other hospitals & 41.3 & 22.8 & 35.5 & TOTAL & 31.8 & 29.7 & 29.3 \\
\hline TOTAL & 38.9 & 39.5 & 41.3 & Far West & & & \\
\hline Northern Sydney & & & & Broken Hill Base & 24.6 & 15.5 & 19.1 \\
\hline Hornsby & 28.8 & 37.6 & 34.5 & TOTAL & 24.6 & 15.5 & 19.1 \\
\hline Manly & 32.8 & 30.7 & 31.9 & Private Hospitals & & & \\
\hline Mona Vale & 46.5 & 43.2 & 42.7 & Mater, North Sydney & 29.7 & 30.7 & 28.2 \\
\hline Royal North Shore & 33.0 & 35.7 & 37.1 & North Shore Private & 27.2 & 30.3 & 30.6 \\
\hline Other hospitals & 0.0 & 0.0 & 0.0 & Sydney Adventist & 35.8 & 32.0 & 33.9 \\
\hline TOTAL & 33.2 & 35.1 & 34.8 & North Gosford Private & 31.8 & 29.9 & 30.3 \\
\hline Central Coast & & & & Hurstville Private & 36.2 & 36.3 & 30.5 \\
\hline Gosford & 36.4 & 38.2 & 39.8 & Kareena Private & 36.3 & 45.0 & 40.6 \\
\hline Wyong & 0.0 & 0.0 & 0.0 & St. George Private & 35.5 & 32.0 & 36.6 \\
\hline TOTAL & 32.9 & 36.8 & 37.5 & Prince of Wales Private & 28.1 & 25.2 & 28.9 \\
\hline Hunter New England & & & & Norwest Private & 46.1 & 47.2 & 45.5 \\
\hline Armidale & 33.3 & 35.5 & 33.7 & Sydney Southwest Private & 33.4 & 36.3 & 35.9 \\
\hline Inverell & 31.4 & 33.3 & 51.9 & Nepean Private & 34.0 & 40.7 & 40.6 \\
\hline Tamworth Base & 37.5 & 44.8 & 48.3 & Westmead Private & 37.2 & 34.3 & 34.4 \\
\hline Manning Base & 23.7 & 33.6 & 37.6 & Figtree Private & 51.0 & 49.8 & 52.3 \\
\hline Maitland & 39.1 & 40.3 & 38.5 & Newcastle Private & 30.1 & 33.3 & 30.3 \\
\hline John Hunter & 28.5 & 34.0 & 34.2 & Calvary, Wagga Wagga & 27.1 & 26.9 & 26.7 \\
\hline Other hospitals & 23.8 & 26.8 & 27.5 & Other hospitals & 33.3 & 37.1 & 36.1 \\
\hline \multirow{2}{*}{ TOTAL } & 30.8 & 35.0 & 36.2 & TOTAL & 33.9 & 33.9 & 34.0 \\
\hline & & & & TOTAL NSW* & 32.8 & 33.0 & 33.8 \\
\hline
\end{tabular}




\section{Outcome of selected primipara.}

\section{Indicator 1.3}

Definition: The number of selected primipara who undergo an instrumental vaginal birth as a percentage of the total number of selected primipara who give birth.

A selected primipara is defined as a woman who is 20-34 years of age at the time of giving birth; giving birth for the first time at greater than 20 weeks gestation; singleton pregnancy; cephalic presentation; and at $37^{\circ}$ to $41^{\circ}$ weeks gestation.

Table 56 shows aggregate information for hospitals where at least 200 mothers gave birth in 2009, totals for hospitals within each Local Health District, and the NSW total.

Table 56. 1.3 Selected primipara who undergo an instrumental vaginal birth by Local Health District and hospital, NSW 2007-2009\#

\begin{tabular}{|c|c|c|c|c|c|c|c|}
\hline Local Health District-hospital & $\begin{array}{c}2007 \\
\%\end{array}$ & $\begin{array}{c}2008 \\
\%\end{array}$ & $\begin{array}{c}2009 \\
\%\end{array}$ & Local Health District-hospital & $\begin{array}{c}2007 \\
\%\end{array}$ & $\begin{array}{c}2008 \\
\%\end{array}$ & $\begin{array}{c}2009 \\
\%\end{array}$ \\
\hline Sydney & & & & Northern NSW & & & \\
\hline Canterbury & 22.1 & 26.1 & 24.0 & Grafton Base & 17.5 & 17.1 & 17.8 \\
\hline Royal Prince Alfred & 22.7 & 20.5 & 22.7 & Lismore Base & 12.4 & 13.2 & 15.8 \\
\hline TOTAL & 22.6 & 21.7 & 23.0 & Murwillumbah & 11.7 & 17.6 & 7.5 \\
\hline South Western Sydney & & & & Tweed Heads & 15.8 & 15.6 & 13.4 \\
\hline Fairfield & 11.9 & 11.2 & 16.6 & Other hospitals & 15.3 & 2.0 & 6.7 \\
\hline Liverpool & 19.8 & 15.6 & 16.7 & TOTAL & 14.4 & 14.5 & 14.2 \\
\hline Campbelltown & 9.7 & 12.8 & 14.2 & Mid North Coast & & & \\
\hline Bankstown-Lidcombe & 21.4 & 20.9 & 21.3 & Coffs Harbour & 16.3 & 18.6 & 23.4 \\
\hline Bowral & 30.5 & 29.2 & 27.9 & Kempsey & 6.1 & 3.3 & 10.2 \\
\hline Other hospitals & 0.0 & 0.0 & 0.0 & Port Macquarie Base & 17.3 & 22.1 & 20.0 \\
\hline TOTAL & 17.0 & 16.0 & 17.5 & Other hospitals & 0.0 & 0.0 & 25.0 \\
\hline South Eastern Sydney & & & & TOTAL & 15.3 & 18.0 & 20.8 \\
\hline St. George & 23.6 & 25.6 & 24.4 & Southern NSW & & & \\
\hline Sutherland & 16.4 & 16.4 & 25.8 & Bega & 10.3 & 25.0 & 8.8 \\
\hline Royal Hospital for Women & 26.0 & 26.1 & 27.1 & Goulburn Base & 18.7 & 26.4 & 32.4 \\
\hline TOTAL & 23.8 & 24.5 & 26.0 & Moruya & 21.5 & 25.4 & 20.8 \\
\hline Illawarra Shoalhaven & & & & Queanbeyan & 18.7 & 23.4 & 26.7 \\
\hline Shoalhaven & 15.7 & 17.1 & 20.0 & Other hospitals & 13.4 & 12.0 & 11.1 \\
\hline Wollongong & 20.3 & 20.2 & 18.1 & TOTAL & 17.1 & 22.2 & 21.5 \\
\hline Other hospitals & 22.6 & 35.0 & 37.5 & Murrumbidgee & & & \\
\hline TOTAL & 19.5 & 19.9 & 19.1 & Griffith Base & 23.6 & 19.2 & 17.8 \\
\hline Western Sydney & & & & Wagga Wagga Base & 20.0 & 22.5 & 20.7 \\
\hline Auburn & 13.7 & 14.9 & 17.9 & Other hospitals & 23.5 & 20.6 & 19.0 \\
\hline Blacktown & 20.0 & 22.5 & 25.7 & TOTAL & 22.3 & 21.0 & 19.4 \\
\hline Westmead & 22.0 & 27.1 & 24.8 & Western NSW & & & \\
\hline TOTAL & 20.0 & 23.9 & 24.1 & Dubbo Base & 17.6 & 17.5 & 17.8 \\
\hline Nepean Blue Mountains & & & & Mudgee & 26.7 & 23.7 & 16.1 \\
\hline Blue Mountains & 22.8 & 18.9 & 17.8 & Bathurst Base & 7.5 & 14.6 & 13.8 \\
\hline Nepean & 22.1 & 22.8 & 21.9 & Orange Base & 21.2 & 20.0 & 23.8 \\
\hline Hawkesbury & 15.2 & 16.3 & 22.6 & Other hospitals & 16.9 & 11.5 & 6.0 \\
\hline Other hospitals & 10.9 & 10.5 & 9.7 & TOTAL & 17.5 & 17.3 & 16.8 \\
\hline TOTAL & 20.4 & 20.9 & 21.5 & Far West & & & \\
\hline Northern Sydney & & & & Broken Hill Base & 12.3 & 14.1 & 10.3 \\
\hline Hornsby & 24.0 & 20.4 & 23.3 & TOTAL & 12.3 & 14.1 & 10.3 \\
\hline Manly & 18.8 & 23.0 & 26.2 & Private Hospitals & & & \\
\hline Mona Vale & 35.0 & 28.1 & 28.1 & Mater, North Sydney & 35.9 & 35.9 & 30.9 \\
\hline Royal North Shore & 19.5 & 18.3 & 23.3 & North Shore Private & 28.1 & 29.2 & 25.9 \\
\hline Other hospitals & 0.0 & 0.0 & 0.0 & Sydney Adventist & 22.1 & 27.8 & 25.2 \\
\hline TOTAL & 22.1 & 20.4 & 23.7 & North Gosford Private & 22.4 & 21.2 & 24.1 \\
\hline Central Coast & & & & Hurstville Private & 29.1 & 34.2 & 24.0 \\
\hline Gosford & 21.0 & 22.1 & 22.6 & Kareena Private & 33.6 & 40.3 & 27.1 \\
\hline Wyong & 10.3 & 4.2 & 0.0 & St. George Private & 29.6 & 34.8 & 32.4 \\
\hline TOTAL & 20.0 & 21.4 & 21.3 & Prince of Wales Private & 24.6 & 23.0 & 26.2 \\
\hline Hunter New England & & & & Norwest Private & 35.6 & 30.2 & 29.4 \\
\hline Armidale & 11.8 & 15.7 & 8.4 & Sydney Southwest Private & 22.1 & 23.7 & 27.1 \\
\hline Inverell & 19.6 & 19.6 & 25.0 & Nepean Private & 26.5 & 27.5 & 22.1 \\
\hline Tamworth Base & 31.3 & 27.9 & 32.0 & Westmead Private & 28.7 & 27.0 & 28.6 \\
\hline Manning Base & 21.6 & 16.4 & 20.4 & Figtree Private & 31.1 & 31.6 & 27.7 \\
\hline Maitland & 19.4 & 16.8 & 18.1 & Newcastle Private & 24.5 & 28.8 & 25.9 \\
\hline John Hunter & 22.2 & 23.1 & 20.0 & Calvary, Wagga Wagga & 33.3 & 29.3 & 33.5 \\
\hline Other hospitals & 17.8 & 14.6 & 12.0 & Other hospitals & 34.8 & 29.0 & 40.3 \\
\hline \multirow[t]{2}{*}{ TOTAL } & 21.4 & 20.0 & 19.5 & TOTAL & 28.2 & 29.3 & 27.6 \\
\hline & & & & TOTAL NSW* & 22.3 & 22.7 & 22.8 \\
\hline
\end{tabular}

Source: NSW Perinatal Data Collection (HOIST). Centre for Epidemiology and Research, NSW Ministry of Health.

\# Hospitals with more than 200 births are identified individually.

* Total NSW includes births at home assisted by independent midwives. 
Outcome of selected primipara.

\section{Indicator 1.4}

Definition: The number of selected primipara undergoing caesarean section birth as a percentage of the total number of selected primipara who give birth.

A selected primipara is defined as a woman who is 20-34 years of age at the time of giving birth; giving birth for the first time at greater than 20 weeks gestation; singleton pregnancy; cephalic presentation; and at $37^{\circ}$ to $41^{\circ}$ weeks gestation.

Table 57 shows aggregate information for hospitals where at least 200 mothers gave birth in 2009, totals for hospitals within each Local Health District, and the NSW total.

Table 57. 1.4 Selected primipara undergoing caesarean section by Local Health District and hospital, NSW 2007-2009\#

\begin{tabular}{|c|c|c|c|c|c|c|c|}
\hline Local Health District-hospital & $\begin{array}{c}2007 \\
\%\end{array}$ & $\begin{array}{c}2008 \\
\%\end{array}$ & $\begin{array}{c}2009 \\
\%\end{array}$ & Local Health District-hospital & $\begin{array}{c}2007 \\
\%\end{array}$ & $\begin{array}{c}2008 \\
\%\end{array}$ & $\begin{array}{c}2009 \\
\%\end{array}$ \\
\hline Sydney & & & & Northern NSW & & & \\
\hline Canterbury & 19.9 & 16.7 & 20.8 & Grafton Base & 25.0 & 27.0 & 24.6 \\
\hline Royal Prince Alfred & 23.3 & 25.5 & 25.4 & Lismore Base & 27.0 & 26.8 & 25.0 \\
\hline TOTAL & 22.6 & 23.6 & 24.4 & Murwillumbah & 26.6 & 18.6 & 5.0 \\
\hline South Western Sydney & & & & Tweed Heads & 19.0 & 17.3 & 18.7 \\
\hline Fairfield & 14.7 & 13.0 & 12.2 & Other hospitals & 0.0 & 0.0 & 0.0 \\
\hline Liverpool & 17.6 & 19.4 & 15.7 & TOTAL & 22.0 & 20.9 & 20.0 \\
\hline Campbelltown & 22.8 & 20.3 & 21.0 & Mid North Coast & & & \\
\hline Bankstown-Lidcombe & 16.5 & 15.3 & 16.5 & Coffs Harbour & 23.5 & 25.3 & 25.5 \\
\hline Bowral & 20.1 & 15.2 & 19.3 & Kempsey & 20.4 & 21.3 & 18.6 \\
\hline Other hospitals & 0.0 & 0.0 & 0.0 & Port Macquarie Base & 20.3 & 20.2 & 21.6 \\
\hline TOTAL & 18.0 & 17.2 & 16.8 & Other hospitals & 0.0 & 0.0 & 0.0 \\
\hline South Eastern Sydney & & & & TOTAL & 21.4 & 22.6 & 23.2 \\
\hline St. George & 18.8 & 23.5 & 20.3 & Southern NSW & & & \\
\hline Sutherland & 17.4 & 13.3 & 16.7 & Bega & 28.2 & 18.8 & 22.8 \\
\hline Royal Hospital for Women & 24.5 & 24.5 & 21.5 & Goulburn Base & 29.3 & 18.1 & 29.6 \\
\hline TOTAL & 21.6 & 22.5 & 20.2 & Moruya & 20.3 & 21.1 & 24.7 \\
\hline Illawarra Shoalhaven & & & & Queanbeyan & 13.3 & 13.0 & 13.3 \\
\hline Shoalhaven & 27.1 & 27.1 & 22.9 & Other hospitals & 19.5 & 17.3 & 13.3 \\
\hline Wollongong & 23.8 & 20.0 & 23.0 & TOTAL & 21.4 & 17.5 & 20.9 \\
\hline Other hospitals & 19.4 & 5.0 & 20.8 & Murrumbidgee & & & \\
\hline TOTAL & 24.3 & 21.2 & 22.9 & Griffith Base & 12.2 & 22.2 & 22.8 \\
\hline Western Sydney & & & & Wagga Wagga Base & 25.7 & 28.4 & 28.2 \\
\hline Auburn & 17.1 & 14.3 & 18.4 & Other hospitals & 27.0 & 26.7 & 29.4 \\
\hline Blacktown & 25.2 & 23.1 & 23.7 & TOTAL & 22.9 & 26.3 & 27.4 \\
\hline Westmead & 20.8 & 22.1 & 24.6 & Western NSW & & & \\
\hline TOTAL & 21.6 & 21.3 & 23.4 & Dubbo Base & 25.1 & 25.0 & 19.9 \\
\hline Nepean Blue Mountains & & & & Mudgee & 16.7 & 18.4 & 19.6 \\
\hline Blue Mountains & 21.5 & 34.0 & 31.1 & Bathurst Base & 27.6 & 18.8 & 26.9 \\
\hline Nepean & 28.9 & 29.7 & 29.2 & Orange Base & 27.7 & 23.6 & 29.6 \\
\hline Hawkesbury & 34.8 & 37.8 & 32.6 & Other hospitals & 26.6 & 23.8 & 34.0 \\
\hline Other hospitals & 26.1 & 29.8 & 25.8 & TOTAL & 25.8 & 22.7 & 25.4 \\
\hline TOTAL & 29.4 & 31.3 & 29.9 & Far West & & & \\
\hline Northern Sydney & & & & Broken Hill Base & 33.8 & 36.6 & 36.8 \\
\hline Hornsby & 23.8 & 30.7 & 23.1 & TOTAL & 33.8 & 36.6 & 36.8 \\
\hline Manly & 17.8 & 22.0 & 24.3 & Private Hospitals & & & \\
\hline Mona Vale & 20.7 & 26.0 & 23.6 & Mater, North Sydney & 34.2 & 35.9 & 39.0 \\
\hline Royal North Shore & 26.0 & 27.0 & 30.1 & North Shore Private & 37.8 & 39.5 & 37.9 \\
\hline Other hospitals & 0.0 & 0.0 & 0.0 & Sydney Adventist & 33.9 & 29.4 & 27.7 \\
\hline TOTAL & 23.0 & 26.0 & 26.0 & North Gosford Private & 43.1 & 48.1 & 46.1 \\
\hline Central Coast & & & & Hurstville Private & 35.1 & 29.1 & 36.1 \\
\hline Gosford & 35.3 & 31.5 & 33.8 & Kareena Private & 44.5 & 38.7 & 37.2 \\
\hline Wyong & 5.9 & 0.0 & 0.0 & St. George Private & 34.8 & 33.9 & 42.0 \\
\hline TOTAL & 32.4 & 30.4 & 31.8 & Prince of Wales Private & 35.5 & 36.0 & 38.3 \\
\hline Hunter New England & & & & Norwest Private & 22.5 & 22.0 & 30.5 \\
\hline Armidale & 16.7 & 24.8 & 18.1 & Sydney Southwest Private & 22.4 & 23.7 & 24.0 \\
\hline Inverell & 37.3 & 41.2 & 32.7 & Nepean Private & 29.4 & 30.4 & 29.9 \\
\hline Tamworth Base & 22.3 & 26.7 & 14.3 & Westmead Private & 27.6 & 32.3 & 32.1 \\
\hline Manning Base & 18.0 & 20.5 & 22.3 & Figtree Private & 26.8 & 22.5 & 34.0 \\
\hline Maitland & 22.5 & 27.3 & 31.6 & Newcastle Private & 33.8 & 29.5 & 28.3 \\
\hline John Hunter & 17.4 & 17.0 & 20.5 & Calvary, Wagga Wagga & 29.9 & 33.5 & 27.3 \\
\hline Other hospitals & 12.8 & 17.2 & 16.7 & Other hospitals & 31.9 & 27.4 & 25.0 \\
\hline TOTAL & 18.7 & 21.1 & 21.9 & TOTAL & 32.7 & 32.6 & 34.2 \\
\hline & & & & TOTAL NSW* & 24.9 & 25.1 & 25.7 \\
\hline
\end{tabular}


Vaginal birth following caesarean section.

\section{Indicator 2.1}

Definition: Total number of women delivering vaginally following a previous primary caesarean section as a percentage of the total number of women delivering who have had a previous primary caesarean section and no intervening pregnancies greater than 20 weeks gestation.
Table 58 shows aggregate information for hospitals where at least 200 mothers gave birth in 2009, totals for hospitals within each Local Health District, and the NSW total.

Table 58. 2.1 Vaginal delivery after previous primary caesarean section by Local Health District and hospital, NSW 2007-2009\#

\begin{tabular}{|c|c|c|c|c|c|c|c|}
\hline Local Health District-hospital & $\begin{array}{c}2007 \\
\%\end{array}$ & $\begin{array}{c}2008 \\
\%\end{array}$ & $\begin{array}{c}2009 \\
\%\end{array}$ & Local Health District-hospital & $\begin{array}{c}2007 \\
\%\end{array}$ & $\begin{array}{c}2008 \\
\%\end{array}$ & $\begin{array}{c}2009 \\
\%\end{array}$ \\
\hline Sydney & & & & Northern NSW & & & \\
\hline Canterbury & 16.9 & 12.0 & 15.6 & Grafton Base & 12.2 & 7.7 & 17.6 \\
\hline Royal Prince Alfred & 13.5 & 11.0 & 14.3 & Lismore Base & 20.1 & 18.4 & 23.0 \\
\hline TOTAL & 14.3 & 11.3 & 14.7 & Murwillumbah & 23.3 & 12.8 & 6.9 \\
\hline South Western Sydney & & & & Tweed Heads & 19.1 & 17.2 & 16.6 \\
\hline Fairfield & 5.4 & 0.0 & 2.8 & Other hospitals & 100.0 & 0.0 & 100.0 \\
\hline Liverpool & 20.5 & 27.7 & 17.1 & TOTAL & 19.7 & 16.2 & 18.9 \\
\hline Campbelltown & 15.6 & 22.6 & 12.5 & Mid North Coast & & & \\
\hline Bankstown-Lidcombe & 11.0 & 22.0 & 12.2 & Coffs Harbour & 10.0 & 14.6 & 14.7 \\
\hline Bowral & 20.7 & 11.9 & 14.3 & Kempsey & 30.4 & 16.7 & 8.7 \\
\hline Other hospitals & 15.3 & 20.2 & 12.9 & Port Macquarie Base & 21.3 & 14.8 & 9.7 \\
\hline TOTAL & 18.0 & 17.2 & 16.8 & Other hospitals & 17.0 & 15.0 & 12.6 \\
\hline South Eastern Sydney & & & & TOTAL & 21.4 & 22.6 & 23.2 \\
\hline St. George & 23.0 & 16.6 & 19.3 & Southern NSW & & & \\
\hline Sutherland & 6.7 & 6.1 & 1.3 & Bega & 9.1 & 18.2 & 38.1 \\
\hline Royal Hospital for Women & 8.3 & 9.8 & 10.7 & Goulburn Base & 26.5 & 30.3 & 22.6 \\
\hline TOTAL & 13.1 & 11.5 & 12.6 & Moruya & 12.5 & 22.5 & 7.4 \\
\hline Illawarra Shoalhaven & & & & Queanbeyan & 5.3 & 16.7 & 14.3 \\
\hline Shoalhaven & 6.3 & 3.4 & 11.0 & Other hospitals & 16.7 & 25.0 & 31.8 \\
\hline Wollongong & 25.3 & 23.4 & 20.9 & TOTAL & 16.1 & 23.7 & 22.1 \\
\hline Other hospitals & 9.1 & 8.3 & 50.0 & Murrumbidgee & & & \\
\hline TOTAL & 18.3 & 16.7 & 18.0 & Griffith Base & 12.8 & 17.6 & 20.0 \\
\hline Western Sydney & & & & Wagga Wagga Base & 8.7 & 13.3 & 11.0 \\
\hline Auburn & 4.0 & 1.6 & 3.1 & Other hospitals & 5.3 & 10.7 & 9.0 \\
\hline Blacktown & 18.4 & 22.5 & 18.0 & TOTAL & 8.3 & 12.9 & 12.4 \\
\hline Westmead & 20.8 & 16.9 & 17.4 & Western NSW & & & \\
\hline TOTAL & 18.7 & 17.6 & 16.6 & Dubbo Base & 25.3 & 21.9 & 12.9 \\
\hline Nepean Blue Mountains & & & & Mudgee & 0.0 & 0.0 & 15.8 \\
\hline Blue Mountains & 0.0 & 0.0 & 0.0 & Bathurst Base & 24.4 & 13.4 & 14.3 \\
\hline Nepean & 14.8 & 16.5 & 17.8 & Orange Base & 8.9 & 12.4 & 12.3 \\
\hline Hawkesbury & 14.1 & 14.7 & 14.6 & Other hospitals & 16.7 & 6.4 & 17.2 \\
\hline Other hospitals & 15.6 & 16.7 & 13.3 & TOTAL & 17.4 & 13.8 & 13.8 \\
\hline TOTAL & 14.3 & 15.7 & 16.0 & Far West & & & \\
\hline Northern Sydney & & & & Broken Hill Base & 6.5 & 13.8 & 13.0 \\
\hline Hornsby & 9.4 & 11.1 & 9.6 & TOTAL & 6.5 & 13.8 & 13.0 \\
\hline Manly & 17.3 & 9.0 & 11.3 & Private Hospitals & & & \\
\hline Mona Vale & 4.2 & 13.0 & 16.0 & Mater, North Sydney & 3.6 & 3.9 & 4.3 \\
\hline Royal North Shore & 11.2 & 9.8 & 9.4 & North Shore Private & 3.8 & 4.2 & 6.2 \\
\hline Other hospitals & 10.4 & 10.6 & 10.3 & Sydney Adventist & 6.4 & 9.2 & 8.5 \\
\hline TOTAL & 23.0 & 26.0 & 26.0 & North Gosford Private & 6.8 & 6.5 & 3.9 \\
\hline Central Coast & & & & Hurstville Private & 2.9 & 2.8 & 6.1 \\
\hline Gosford & 5.7 & 8.6 & 7.0 & Kareena Private & 1.9 & 0.0 & 3.0 \\
\hline Wyong & 100.0 & 0.0 & 0.0 & St. George Private & 6.4 & 6.5 & 4.0 \\
\hline TOTAL & 6.3 & 8.6 & 7.0 & Prince of Wales Private & 4.8 & 4.3 & 3.4 \\
\hline Hunter New England & & & & Norwest Private & 11.7 & 10.8 & 11.4 \\
\hline Armidale & 16.3 & 27.8 & 15.4 & Sydney Southwest Private & 5.1 & 5.9 & 3.3 \\
\hline Inverell & 13.5 & 14.3 & 9.1 & Nepean Private & 10.4 & 11.5 & 5.8 \\
\hline Tamworth Base & 13.1 & 8.2 & 23.8 & Westmead Private & 8.3 & 5.1 & 8.3 \\
\hline Manning Base & 29.5 & 27.7 & 10.8 & Figtree Private & 6.4 & 7.1 & 5.9 \\
\hline Maitland & 16.4 & 17.1 & 19.4 & Newcastle Private & 9.3 & 11.4 & 12.4 \\
\hline John Hunter & 21.7 & 27.3 & 23.7 & Calvary, Wagga Wagga & 14.3 & 7.2 & 12.5 \\
\hline Other hospitals & 20.0 & 14.4 & 14.7 & Other hospitals & 2.0 & 7.8 & 8.5 \\
\hline \multirow[t]{2}{*}{ TOTAL } & 19.6 & 21.5 & 19.8 & TOTAL & 6.2 & 6.3 & 6.5 \\
\hline & & & & TOTAL NSW* & 12.3 & 12.5 & 12.0 \\
\hline
\end{tabular}


Major perineal tears and surgical repair of the perineum. Indicator 3.1

Definition: Total number of selected primipara with an intact perineum as a percentage of the total number of selected primipara delivering vaginally.

A selected primipara is defined as a woman who is 20-34 years of age at the time of giving birth; giving birth for the first time at greater than 20 weeks gestation; singleton pregnancy; cephalic presentation; and at $37^{\circ}$ to $41^{\circ}$ weeks gestation.

Table 59 shows aggregate information for hospitals where at least 200 mothers gave birth in 2009, totals for hospitals within each Local Health District, and the NSW total.

Table 59. 3.1 Selected primipara delivering vaginally with an intact perineum by Local Health District and hospital, NSW 2007-2009\#

\begin{tabular}{|c|c|c|c|c|c|c|c|}
\hline Local Health District-hospital & $\begin{array}{c}2007 \\
\%\end{array}$ & $\begin{array}{c}2008 \\
\%\end{array}$ & $\begin{array}{c}2009 \\
\%\end{array}$ & Local Health District-hospital & $\begin{array}{c}2007 \\
\%\end{array}$ & $\begin{array}{c}2008 \\
\%\end{array}$ & $\begin{array}{c}2009 \\
\%\end{array}$ \\
\hline Sydney & & & & Northern NSW & & & \\
\hline Canterbury & 5.0 & 3.2 & 5.0 & Grafton Base & 16.7 & 23.5 & 19.1 \\
\hline Royal Prince Alfred & 4.7 & 6.5 & 5.0 & Lismore Base & 11.7 & 11.8 & 8.9 \\
\hline TOTAL & 4.8 & 5.7 & 5.0 & Murwillumbah & 21.7 & 27.7 & 26.3 \\
\hline South Western Sydney & & & & Tweed Heads & 20.8 & 13.6 & 10.8 \\
\hline Fairfield & 10.0 & 14.5 & 11.6 & Other hospitals & 16.9 & 13.7 & 17.8 \\
\hline Liverpool & 7.5 & 9.4 & 8.4 & TOTAL & 17.2 & 15.6 & 12.3 \\
\hline Campbelltown & 8.1 & 12.9 & 16.5 & Mid North Coast & & & \\
\hline Bankstown-Lidcombe & 5.5 & 5.3 & 8.2 & Coffs Harbour & 18.9 & 17.8 & 11.0 \\
\hline Bowral & 15.9 & 14.5 & 15.0 & Kempsey & 25.6 & 17.0 & 16.7 \\
\hline Other hospitals & 0.0 & 0.0 & 100.0 & Port Macquarie Base & 20.4 & 19.9 & 20.0 \\
\hline TOTAL & 8.3 & 10.7 & 11.5 & Other hospitals & 30.0 & 28.6 & 0.0 \\
\hline South Eastern Sydney & & & & TOTAL & 20.5 & 18.7 & 14.7 \\
\hline St. George & 11.6 & 9.6 & 8.6 & Southern NSW & & & \\
\hline Sutherland & 16.1 & 10.9 & 10.9 & Bega & 17.9 & 23.1 & 27.3 \\
\hline Royal Hospital for Women & 9.1 & 7.1 & 6.2 & Goulburn Base & 13.2 & 3.4 & 8.0 \\
\hline TOTAL & 11.0 & 8.5 & 7.9 & Moruya & 22.2 & 19.6 & 22.4 \\
\hline Illawarra Shoalhaven & & & & Queanbeyan & 15.4 & 17.9 & 21.8 \\
\hline Shoalhaven & 9.9 & 15.2 & 8.9 & Other hospitals & 25.8 & 32.8 & 38.5 \\
\hline Wollongong & 6.7 & 12.5 & 13.9 & TOTAL & 19.3 & 19.1 & 22.7 \\
\hline Other hospitals & 28.0 & 10.5 & 15.8 & Murrumbidgee & & & \\
\hline TOTAL & 8.1 & 13.0 & 12.7 & Griffith Base & 13.9 & 18.2 & 11.5 \\
\hline Western Sydney & & & & Wagga Wagga Base & 18.5 & 19.0 & 14.4 \\
\hline Auburn & 13.6 & 7.7 & 11.1 & Other hospitals & 25.5 & 19.0 & 21.7 \\
\hline Blacktown & 6.6 & 5.5 & 5.7 & TOTAL & 19.9 & 18.8 & 16.4 \\
\hline Westmead & 5.2 & 6.5 & 5.2 & Western NSW & & & \\
\hline TOTAL & 7.1 & 6.4 & 6.2 & Dubbo Base & 13.4 & 13.3 & 21.4 \\
\hline Nepean Blue Mountains & & & & Mudgee & 6.0 & 16.1 & 28.9 \\
\hline Blue Mountains & 4.8 & 11.4 & 12.9 & Bathurst Base & 19.6 & 12.8 & 17.1 \\
\hline Nepean & 8.0 & 7.9 & 7.9 & Orange Base & 18.7 & 16.1 & 12.8 \\
\hline Hawkesbury & 13.3 & 10.0 & 5.6 & Other hospitals & 18.7 & 11.8 & 13.6 \\
\hline Other hospitals & 23.5 & 20.0 & 8.7 & TOTAL & 16.0 & 14.0 & 18.3 \\
\hline TOTAL & 9.3 & 8.9 & 7.7 & Far West & & & \\
\hline Northern Sydney & & & & Broken Hill Base & 23.3 & 22.2 & 25.6 \\
\hline Hornsby & 8.2 & 7.1 & 6.4 & TOTAL & 23.3 & 22.2 & 25.6 \\
\hline Manly & 8.1 & 6.6 & 8.3 & Private Hospitals & & & \\
\hline Mona Vale & 14.0 & 12.0 & 14.7 & Mater, North Sydney & 7.3 & 7.6 & 7.0 \\
\hline Royal North Shore & 9.2 & 7.5 & 5.3 & North Shore Private & 10.3 & 6.3 & 12.6 \\
\hline Other hospitals & 8.0 & 17.8 & 9.1 & Sydney Adventist & 7.1 & 6.6 & 5.4 \\
\hline TOTAL & 9.4 & 8.2 & 6.9 & North Gosford Private & 16.6 & 20.8 & 20.3 \\
\hline Central Coast & & & & Hurstville Private & 4.1 & 6.1 & 9.7 \\
\hline Gosford & 8.4 & 6.8 & 5.3 & Kareena Private & 4.9 & 14.5 & 10.0 \\
\hline Wyong & 7.8 & 0.0 & 9.3 & St. George Private & 12.9 & 6.2 & 7.5 \\
\hline TOTAL & 8.4 & 6.4 & 5.7 & Prince of Wales Private & 12.5 & 12.1 & 13.3 \\
\hline Hunter New England & & & & Norwest Private & 9.8 & 12.6 & 12.5 \\
\hline Armidale & 20.0 & 16.5 & 14.7 & Sydney Southwest Private & 12.0 & 12.0 & 6.8 \\
\hline Inverell & 15.6 & 20.0 & 14.3 & Nepean Private & 5.4 & 11.6 & 10.7 \\
\hline Tamworth Base & 13.8 & 4.8 & 7.5 & Westmead Private & 7.1 & 7.8 & 9.7 \\
\hline Manning Base & 19.3 & 11.2 & 13.1 & Figtree Private & 5.9 & 10.7 & 10.1 \\
\hline Maitland & 9.0 & 9.3 & 6.5 & Newcastle Private & 11.8 & 17.6 & 18.4 \\
\hline John Hunter & 6.9 & 5.0 & 6.0 & Calvary, Wagga Wagga & 10.9 & 11.7 & 4.7 \\
\hline Other hospitals & 16.2 & 13.8 & 8.8 & Other hospitals & 8.7 & 6.7 & 11.1 \\
\hline \multirow[t]{2}{*}{ TOTAL } & 11.1 & 8.5 & 7.7 & TOTAL & 9.4 & 10.0 & 10.4 \\
\hline & & & & TOTAL NSW* & 10.0 & 9.8 & 9.6 \\
\hline
\end{tabular}

Source: NSW Perinatal Data Collection (HOIST). Centre for Epidemiology and Research, NSW Ministry of Health.

\# Hospitals with more than 200 births are identified individually.

* Total NSW includes births at home assisted by independent midwives. 
Major perineal tears and surgical repair of the perineum.

\section{Indicator 3.2}

Definition: Total number of selected primipara undergoing episiotomy and no perineal tear while giving birth vaginally as a percentage of the total number of selected primipara delivering vaginally.

A selected primipara is defined as a woman who is 20-34 years of age at the time of giving birth; giving birth for the first time at greater than 20 weeks gestation; singleton pregnancy; cephalic presentation; and at $37^{\circ}$ to $41^{\circ}$ weeks gestation.

Table 60 shows aggregate information for hospitals where at least 200 mothers gave birth in 2009, totals for hospitals within each Local Health District, and the NSW total.

Table 60. 3.2 Selected primipara undergoing episiotomy and no perineal tear by Local Health District and hospital, NSW 2007-2009\#

\begin{tabular}{|c|c|c|c|c|c|c|c|}
\hline Local Health District-hospital & $\begin{array}{c}2007 \\
\%\end{array}$ & $\begin{array}{c}2008 \\
\%\end{array}$ & $\begin{array}{c}2009 \\
\%\end{array}$ & Local Health District-hospital & $\begin{array}{c}2007 \\
\%\end{array}$ & $\begin{array}{c}2008 \\
\%\end{array}$ & $\begin{array}{c}2009 \\
\%\end{array}$ \\
\hline Sydney & & & & Northern NSW & & & \\
\hline Canterbury & 17.3 & 19.8 & 29.8 & Grafton Base & 18.9 & 21.0 & 22.5 \\
\hline Royal Prince Alfred & 16.6 & 17.4 & 16.6 & Lismore Base & 17.5 & 12.2 & 18.1 \\
\hline TOTAL & 16.8 & 17.9 & 19.7 & Murwillumbah & 8.7 & 16.9 & 10.5 \\
\hline South Western Sydney & & & & Tweed Heads & 18.5 & 14.0 & 14.9 \\
\hline Fairfield & 32.6 & 21.2 & 30.5 & Other hospitals & 11.9 & 7.8 & 15.6 \\
\hline Liverpool & 33.5 & 31.3 & 28.7 & TOTAL & 16.8 & 14.1 & 16.6 \\
\hline Campbelltown & 16.9 & 19.2 & 20.3 & Mid North Coast & & & \\
\hline Bankstown-Lidcombe & 29.2 & 30.2 & 32.3 & Coffs Harbour & 11.2 & 15.0 & 24.3 \\
\hline Bowral & 13.0 & 15.9 & 18.6 & Kempsey & 7.7 & 8.5 & 4.2 \\
\hline Other hospitals & 0.0 & 0.0 & 0.0 & Port Macquarie Base & 17.2 & 19.9 & 16.6 \\
\hline TOTAL & 27.7 & 25.1 & 27.2 & Other hospitals & 0.0 & 14.3 & 0.0 \\
\hline South Eastern Sydney & & & & TOTAL & 13.1 & 16.2 & 18.9 \\
\hline St. George & 14.1 & 14.5 & 19.4 & Southern NSW & & & \\
\hline Sutherland & 9.3 & 8.0 & 16.8 & Bega & 10.7 & 7.7 & 13.6 \\
\hline Royal Hospital for Women & 26.3 & 27.5 & 27.0 & Goulburn Base & 50.9 & 66.1 & 74.0 \\
\hline TOTAL & 19.6 & 20.0 & 22.6 & Moruya & 15.9 & 19.6 & 20.7 \\
\hline Illawarra Shoalhaven & & & & Queanbeyan & 10.8 & 7.5 & 10.3 \\
\hline Shoalhaven & 18.2 & 18.2 & 18.4 & Other hospitals & 7.6 & 11.5 & 2.6 \\
\hline Wollongong & 18.5 & 12.7 & 14.1 & TOTAL & 18.9 & 23.0 & 23.8 \\
\hline Other hospitals & 8.0 & 10.5 & 10.5 & Murrumbidgee & & & \\
\hline TOTAL & 18.1 & 13.8 & 15.0 & Griffith Base & 23.1 & 20.8 & 20.5 \\
\hline Western Sydney & & & & Wagga Wagga Base & 18.5 & 19.0 & 20.0 \\
\hline Auburn & 19.2 & 19.3 & 24.8 & Other hospitals & 22.1 & 21.5 & 27.0 \\
\hline Blacktown & 32.8 & 26.1 & 26.6 & TOTAL & 21.2 & 20.4 & 22.6 \\
\hline Westmead & 34.3 & 36.6 & 34.7 & Western NSW & & & \\
\hline TOTAL & 31.2 & 30.6 & 30.7 & Dubbo Base & 32.2 & 34.0 & 23.1 \\
\hline Nepean Blue Mountains & & & & Mudgee & 30.0 & 24.2 & 8.9 \\
\hline Blue Mountains & 8.1 & 11.4 & 9.7 & Bathurst Base & 8.2 & 17.1 & 20.5 \\
\hline Nepean & 25.0 & 24.9 & 21.3 & Orange Base & 14.5 & 14.3 & 15.0 \\
\hline Hawkesbury & 14.7 & 16.2 & 22.4 & Other hospitals & 17.6 & 12.9 & 7.6 \\
\hline Other hospitals & 32.4 & 25.0 & 30.4 & TOTAL & 21.1 & 21.5 & 18.0 \\
\hline TOTAL & 22.3 & 22.9 & 21.3 & Far West & & & \\
\hline Northern Sydney & & & & Broken Hill Base & 16.3 & 17.8 & 16.3 \\
\hline Hornsby & 22.3 & 18.6 & 21.5 & TOTAL & 16.3 & 17.8 & 16.3 \\
\hline Manly & 11.2 & 17.8 & 14.4 & Private Hospitals & & & \\
\hline Mona Vale & 10.5 & 9.9 & 8.8 & Mater, North Sydney & 40.5 & 45.2 & 43.6 \\
\hline Royal North Shore & 13.4 & 20.9 & 24.0 & North Shore Private & 42.9 & 46.8 & 38.7 \\
\hline Other hospitals & 0.0 & 2.2 & 0.0 & Sydney Adventist & 37.3 & 36.9 & 34.6 \\
\hline TOTAL & 14.4 & 17.7 & 19.6 & North Gosford Private & 31.7 & 25.8 & 25.2 \\
\hline Central Coast & & & & Hurstville Private & 43.9 & 44.2 & 36.1 \\
\hline Gosford & 11.4 & 9.8 & 13.2 & Kareena Private & 45.7 & 38.5 & 32.3 \\
\hline Wyong & 7.8 & 4.2 & 4.7 & St. George Private & 24.7 & 36.0 & 32.0 \\
\hline TOTAL & 10.9 & 9.5 & 12.5 & Prince of Wales Private & 34.7 & 32.9 & 34.4 \\
\hline Hunter New England & & & & Norwest Private & 39.2 & 33.3 & 27.5 \\
\hline Armidale & 20.0 & 17.6 & 19.1 & Sydney Southwest Private & 43.1 & 45.6 & 46.8 \\
\hline Inverell & 53.1 & 46.7 & 48.6 & Nepean Private & 52.4 & 48.9 & 47.7 \\
\hline Tamworth Base & 31.0 & 31.7 & 17.8 & Westmead Private & 51.2 & 50.9 & 49.8 \\
\hline Manning Base & 10.5 & 12.9 & 12.3 & Figtree Private & 48.9 & 51.5 & 53.5 \\
\hline Maitland & 14.4 & 10.3 & 12.2 & Newcastle Private & 32.2 & 26.0 & 22.4 \\
\hline John Hunter & 11.1 & 13.4 & 14.9 & Calvary, Wagga Wagga & 27.7 & 20.7 & 29.7 \\
\hline Other hospitals & 16.5 & 11.9 & 11.6 & Other hospitals & 30.4 & 48.9 & 44.4 \\
\hline TOTAL & 15.8 & 14.8 & 15.0 & TOTAL & 39.7 & 40.0 & 37.8 \\
\hline & & & & TOTAL NSW* & 24.9 & 25.0 & 25.4 \\
\hline
\end{tabular}


Major perineal tears and surgical repair of the perineum.

\section{Indicator 3.3}

Definition: Total number of selected primipara sustaining a perineal tear and no episiotomy as a percentage of the total number of selected primipara delivering vaginally.

A selected primipara is defined as a woman who is 20-34 years of age at the time of giving birth; giving birth for the first time at greater than 20 weeks gestation; singleton pregnancy; cephalic presentation; and at $37^{\circ}$ to $41^{\circ}$ weeks gestation.

Table 61 shows aggregate information for hospitals where at least 200 mothers gave birth in 2009, totals for hospitals within each Local Health District, and the NSW total.

Table 61. 3.3 Selected primipara sustaining a perineal tear and no episiotomy by Local Health District and hospital, NSW 2007-2009\#

\begin{tabular}{|c|c|c|c|c|c|c|c|}
\hline Local Health District-hospital & $\begin{array}{c}2007 \\
\%\end{array}$ & $\begin{array}{c}2008 \\
\%\end{array}$ & $\begin{array}{c}2009 \\
\%\end{array}$ & Local Health District-hospital & $\begin{array}{c}2007 \\
\%\end{array}$ & $\begin{array}{c}2008 \\
\%\end{array}$ & $\begin{array}{c}2009 \\
\%\end{array}$ \\
\hline Sydney & & & & Northern NSW & & & \\
\hline Canterbury & 65.7 & 63.7 & 54.9 & Grafton Base & 53.3 & 49.4 & 51.7 \\
\hline Royal Prince Alfred & 70.6 & 69.0 & 72.4 & Lismore Base & 64.6 & 66.8 & 68.4 \\
\hline TOTAL & 69.5 & 67.8 & 68.3 & Murwillumbah & 59.4 & 48.2 & 55.3 \\
\hline South Western Sydney & & & & Tweed Heads & 54.5 & 62.8 & 66.1 \\
\hline Fairfield & 52.3 & 60.2 & 52.7 & Other hospitals & 64.4 & 76.5 & 64.4 \\
\hline Liverpool & 51.1 & 49.0 & 51.5 & TOTAL & 58.7 & 61.9 & 64.5 \\
\hline Campbelltown & 68.2 & 60.0 & 56.4 & Mid North Coast & & & \\
\hline Bankstown-Lidcombe & 57.2 & 57.8 & 49.1 & Coffs Harbour & 53.8 & 57.7 & 51.9 \\
\hline Bowral & 67.4 & 62.1 & 63.7 & Kempsey & 66.7 & 70.2 & 70.8 \\
\hline Other hospitals & 0.0 & 0.0 & 0.0 & Port Macquarie Base & 54.8 & 54.8 & 57.2 \\
\hline TOTAL & 57.2 & 56.7 & 53.0 & Other hospitals & 70.0 & 57.1 & 100.0 \\
\hline South Eastern Sydney & & & & TOTAL & 56.0 & 58.0 & 56.5 \\
\hline St. George & 64.4 & 64.6 & 59.8 & Southern NSW & & & \\
\hline Sutherland & 70.5 & 73.7 & 66.2 & Bega & 67.9 & 64.1 & 47.7 \\
\hline Royal Hospital for Women & 57.4 & 58.2 & 58.4 & Goulburn Base & 28.3 & 22.0 & 14.0 \\
\hline TOTAL & 61.8 & 62.9 & 60.3 & Moruya & 58.7 & 51.8 & 50.0 \\
\hline Illawarra Shoalhaven & & & & Queanbeyan & 69.2 & 68.7 & 59.0 \\
\hline Shoalhaven & 66.1 & 59.1 & 62.0 & Other hospitals & 59.1 & 55.7 & 59.0 \\
\hline Wollongong & 70.6 & 68.4 & 64.9 & TOTAL & 56.4 & 52.1 & 46.8 \\
\hline Other hospitals & 44.0 & 57.9 & 63.2 & Murrumbidgee & & & \\
\hline TOTAL & 68.7 & 66.2 & 64.2 & Griffith Base & 58.3 & 50.6 & 62.8 \\
\hline Western Sydney & & & & Wagga Wagga Base & 52.3 & 52.9 & 57.6 \\
\hline Auburn & 55.5 & 63.3 & 53.7 & Other hospitals & 47.0 & 50.4 & 43.5 \\
\hline Blacktown & 49.2 & 57.7 & 56.5 & TOTAL & 51.9 & 51.4 & 53.8 \\
\hline Westmead & 47.8 & 45.4 & 46.4 & Western NSW & & & \\
\hline TOTAL & 49.5 & 52.0 & 50.7 & Dubbo Base & 45.0 & 43.1 & 45.0 \\
\hline Nepean Blue Mountains & & & & Mudgee & 56.0 & 53.2 & 51.1 \\
\hline Blue Mountains & 82.3 & 65.7 & 71.0 & Bathurst Base & 62.9 & 64.1 & 56.4 \\
\hline Nepean & 54.9 & 58.7 & 59.6 & Orange Base & 50.6 & 58.9 & 51.9 \\
\hline Hawkesbury & 63.3 & 64.6 & 57.8 & Other hospitals & 53.8 & 63.4 & 68.2 \\
\hline Other hospitals & 41.2 & 45.0 & 56.5 & TOTAL & 51.7 & 55.3 & 51.9 \\
\hline TOTAL & 57.8 & 59.3 & 59.6 & Far West & & & \\
\hline Northern Sydney & & & & Broken Hill Base & 58.1 & 57.8 & 53.5 \\
\hline Hornsby & 59.3 & 62.1 & 64.6 & TOTAL & 58.1 & 57.8 & 53.5 \\
\hline Manly & 72.9 & 67.2 & 63.7 & Private Hospitals & & & \\
\hline Mona Vale & 68.6 & 71.1 & 73.5 & Mater, North Sydney & 37.4 & 34.4 & 36.6 \\
\hline Royal North Shore & 65.0 & 58.8 & 53.3 & North Shore Private & 41.8 & 44.9 & 46.4 \\
\hline Other hospitals & 92.0 & 80.0 & 90.9 & Sydney Adventist & 47.7 & 52.4 & 56.8 \\
\hline TOTAL & 66.2 & 63.6 & 60.8 & North Gosford Private & 41.4 & 44.2 & 44.7 \\
\hline Central Coast & & & & Hurstville Private & 39.8 & 36.0 & 43.5 \\
\hline Gosford & 65.8 & 73.4 & 68.0 & Kareena Private & 45.7 & 43.6 & 54.6 \\
\hline Wyong & 79.7 & 87.5 & 79.1 & St. George Private & 56.7 & 53.9 & 56.2 \\
\hline TOTAL & 67.7 & 74.1 & 68.9 & Prince of Wales Private & 47.9 & 47.1 & 40.1 \\
\hline Hunter New England & & & & Norwest Private & 41.2 & 45.2 & 50.2 \\
\hline Armidale & 45.9 & 52.7 & 60.3 & Sydney Southwest Private & 38.2 & 35.1 & 36.8 \\
\hline Inverell & 25.0 & 30.0 & 28.6 & Nepean Private & 36.3 & 32.6 & 29.9 \\
\hline Tamworth Base & 47.1 & 54.0 & 67.2 & Westmead Private & 39.9 & 39.5 & 39.3 \\
\hline Manning Base & 59.6 & 69.8 & 67.2 & Figtree Private & 41.6 & 34.7 & 35.4 \\
\hline Maitland & 72.6 & 73.5 & 73.1 & Newcastle Private & 55.4 & 54.2 & 57.5 \\
\hline John Hunter & 69.4 & 69.9 & 69.1 & Calvary, Wagga Wagga & 47.5 & 48.6 & 50.8 \\
\hline Other hospitals & 59.2 & 64.6 & 73.5 & Other hospitals & 52.2 & 44.4 & 27.8 \\
\hline \multirow[t]{2}{*}{ TOTAL } & 63.4 & 66.8 & 68.8 & TOTAL & 44.3 & 44.0 & 45.2 \\
\hline & & & & TOTAL NSW* & 56.6 & 57.0 & 56.2 \\
\hline
\end{tabular}


Major perineal tears and surgical repair of the perineum.

\section{Indicator 3.4}

Definition: Total number of selected primipara undergoing episiotomy and sustaining a perineal tear while giving birth vaginally as a percentage of the total number of selected primipara delivering vaginally.

A selected primipara is defined as a woman who is 20-34 years of age at the time of giving birth; giving birth for the first time at greater than 20 weeks gestation; singleton pregnancy; cephalic presentation; and at $37^{\circ}$ to $41^{\circ}$ weeks gestation.

Table 62 shows aggregate information for hospitals where at least 200 mothers gave birth in 2009, totals for hospitals within each Local Health District, and the NSW total.

Table 62. 3.4 Selected primipara undergoing episiotomy and sustaining a perineal tear by Local Health District and hospital, NSW 2007-2009\#

\begin{tabular}{|c|c|c|c|c|c|c|c|}
\hline Local Health District-hospital & $\begin{array}{c}2007 \\
\%\end{array}$ & $\begin{array}{c}2008 \\
\%\end{array}$ & $\begin{array}{c}2009 \\
\%\end{array}$ & Local Health District-hospital & $\begin{array}{c}2007 \\
\%\end{array}$ & $\begin{array}{c}2008 \\
\%\end{array}$ & $\begin{array}{c}2009 \\
\%\end{array}$ \\
\hline Sydney & & & & Northern NSW & & & \\
\hline Canterbury & 7.77 & 7.41 & 5.51 & Grafton Base & 4.44 & 1.23 & 0.00 \\
\hline Royal Prince Alfred & 5.42 & 4.87 & 3.47 & Lismore Base & 5.00 & 5.88 & 3.38 \\
\hline TOTAL & 5.95 & 5.45 & 3.94 & Murwillumbah & 1.45 & 3.61 & 2.63 \\
\hline South Western Sydney & & & & Tweed Heads & 1.98 & 3.65 & 2.44 \\
\hline Fairfield & 3.73 & 1.87 & 3.05 & Other hospitals & 3.39 & 0.00 & 2.22 \\
\hline Liverpool & 4.07 & 5.58 & 5.49 & TOTAL & 3.29 & 3.85 & 2.44 \\
\hline Campbelltown & 2.32 & 2.62 & 2.08 & Mid North Coast & & & \\
\hline Bankstown-Lidcombe & 4.82 & 5.13 & 6.57 & Coffs Harbour & 5.92 & 2.82 & 7.14 \\
\hline Bowral & 0.00 & 2.07 & 1.77 & Kempsey & 0.00 & 4.26 & 6.25 \\
\hline Other hospitals & 0.00 & 0.00 & 0.00 & Port Macquarie Base & 2.55 & 3.61 & 6.21 \\
\hline TOTAL & 3.53 & 3.78 & 4.16 & Other hospitals & 0.00 & 0.00 & 0.00 \\
\hline South Eastern Sydney & & & & TOTAL & 3.73 & 3.23 & 6.63 \\
\hline St. George & 8.24 & 9.95 & 11.42 & Southern NSW & & & \\
\hline Sutherland & 2.80 & 3.24 & 4.83 & Bega & 0.00 & 2.56 & 6.82 \\
\hline Royal Hospital for Women & 6.12 & 6.18 & 6.94 & Goulburn Base & 1.89 & 0.00 & 4.00 \\
\hline TOTAL & 6.33 & 6.88 & 7.94 & Moruya & 3.17 & 5.36 & 0.00 \\
\hline Illawarra Shoalhaven & & & & Queanbeyan & 3.08 & 4.48 & 6.41 \\
\hline Shoalhaven & 3.31 & 5.30 & 6.96 & Other hospitals & 3.03 & 0.00 & 0.00 \\
\hline Wollongong & 2.92 & 5.37 & 5.71 & TOTAL & 2.55 & 2.48 & 3.72 \\
\hline Other hospitals & 12.00 & 15.79 & 10.53 & Murrumbidgee & & & \\
\hline TOTAL & 3.35 & 5.66 & 6.15 & Griffith Base & 0.93 & 2.60 & 3.85 \\
\hline Western Sydney & & & & Wagga Wagga Base & 4.62 & 4.96 & 8.00 \\
\hline Auburn & 10.62 & 9.32 & 8.25 & Other hospitals & 4.03 & 4.96 & 4.35 \\
\hline Blacktown & 10.82 & 10.17 & 10.73 & TOTAL & 3.36 & 4.39 & 5.66 \\
\hline Westmead & 11.10 & 10.44 & 11.63 & Western NSW & & & \\
\hline TOTAL & 10.94 & 10.18 & 10.85 & Dubbo Base & 6.93 & 4.26 & 6.11 \\
\hline Nepean Blue Mountains & & & & Mudgee & 2.00 & 4.84 & 11.11 \\
\hline Blue Mountains & 3.23 & 5.71 & 6.45 & Bathurst Base & 4.12 & 2.56 & 1.71 \\
\hline Nepean & 10.19 & 6.90 & 9.69 & Orange Base & 7.23 & 8.33 & 17.29 \\
\hline Hawkesbury & 8.00 & 6.92 & 13.04 & Other hospitals & 6.59 & 6.45 & 6.06 \\
\hline Other hospitals & 2.94 & 5.00 & 4.35 & TOTAL & 6.11 & 5.41 & 8.14 \\
\hline TOTAL & 9.04 & 6.76 & 10.07 & Far West & & & \\
\hline Northern Sydney & & & & Broken Hill Base & 2.33 & 0.00 & 0.00 \\
\hline Hornsby & 7.54 & 11.90 & 6.06 & TOTAL & 2.33 & 0.00 & 0.00 \\
\hline Manly & 5.81 & 6.64 & 12.59 & Private Hospitals & & & \\
\hline Mona Vale & 5.81 & 6.34 & 2.94 & Mater, North Sydney & 11.01 & 7.62 & 7.95 \\
\hline Royal North Shore & 11.32 & 11.32 & 16.44 & North Shore Private & 1.88 & 0.63 & 0.90 \\
\hline Other hospitals & 0.00 & 0.00 & 0.00 & Sydney Adventist & 7.84 & 2.73 & 2.59 \\
\hline TOTAL & 8.47 & 9.44 & 11.69 & North Gosford Private & 9.66 & 5.83 & 4.88 \\
\hline Central Coast & & & & Hurstville Private & 9.94 & 8.63 & 7.41 \\
\hline Gosford & 11.66 & 8.41 & 11.94 & Kareena Private & 1.23 & 1.71 & 0.77 \\
\hline Wyong & 4.69 & 0.00 & 4.65 & St. George Private & 3.87 & 1.04 & 0.86 \\
\hline TOTAL & 10.71 & 7.96 & 11.33 & Prince of Wales Private & 2.20 & 3.62 & 6.41 \\
\hline Hunter New England & & & & Norwest Private & 4.05 & 3.74 & 4.31 \\
\hline Armidale & 5.88 & 10.99 & 5.88 & Sydney Southwest Private & 2.62 & 3.86 & 5.20 \\
\hline Inverell & 6.25 & 3.33 & 8.57 & Nepean Private & 1.79 & 2.11 & 1.52 \\
\hline Tamworth Base & 6.32 & 9.52 & 7.47 & Westmead Private & 0.73 & 0.38 & 0.52 \\
\hline Manning Base & 3.51 & 4.31 & 4.92 & Figtree Private & 0.90 & 1.53 & 0.51 \\
\hline Maitland & 3.34 & 5.15 & 7.89 & Newcastle Private & 0.00 & 0.50 & 0.25 \\
\hline John Hunter & 11.08 & 10.54 & 9.14 & Calvary, Wagga Wagga & 7.92 & 9.91 & 13.28 \\
\hline Other hospitals & 5.77 & 5.77 & 3.72 & Other hospitals & 8.70 & 0.00 & 9.26 \\
\hline \multirow[t]{2}{*}{ TOTAL } & 7.51 & 8.22 & 7.57 & TOTAL & 4.13 & 2.92 & 3.40 \\
\hline & & & & TOTAL NSW* & 6.02 & 5.70 & 6.36 \\
\hline
\end{tabular}


Major perineal tears and surgical repair of the perineum.

\section{Indicator 3.5}

Definition: Total number of selected primipara undergoing surgical repair of the perineum for third degree tear as a percentage of the total number of selected primipara delivering vaginally.

A selected primipara is defined as a woman who is 20-34 years of age at the time of giving birth; giving birth for the first time at greater than 20 weeks gestation; singleton pregnancy; cephalic presentation; and at $37^{\circ}$ to $41^{\circ}$ weeks gestation.

Table 63 shows aggregate information for hospitals where at least 200 mothers gave birth in 2009, totals for hospitals within each Local Health District, and the NSW total.

Table 63. 3.5 Selected primipara undergoing surgical repair of the perineum for third degree tear by Local Health District and hospital, NSW 2007-2009\#

\begin{tabular}{|c|c|c|c|c|c|c|c|}
\hline Local Health District-hospital & $\begin{array}{c}2007 \\
\%\end{array}$ & $\begin{array}{c}2008 \\
\%\end{array}$ & $\begin{array}{c}2009 \\
\%\end{array}$ & Local Health District-hospital & $\begin{array}{c}2007 \\
\%\end{array}$ & $\begin{array}{c}2008 \\
\%\end{array}$ & $\begin{array}{c}2009 \\
\%\end{array}$ \\
\hline Sydney & & & & Northern NSW & & & \\
\hline Canterbury & 9.02 & 6.91 & 2.51 & Grafton Base & 3.33 & 1.23 & 4.49 \\
\hline Royal Prince Alfred & 7.84 & 5.24 & 6.04 & Lismore Base & 2.92 & 3.36 & 3.38 \\
\hline TOTAL & 8.11 & 5.62 & 5.22 & Murwillumbah & 1.45 & 3.61 & 2.63 \\
\hline South Western Sydney & & & & Tweed Heads & 4.29 & 5.65 & 7.05 \\
\hline Fairfield & 5.60 & 4.15 & 4.77 & Other hospitals & 0.00 & 1.96 & 0.00 \\
\hline Liverpool & 3.74 & 5.74 & 6.19 & TOTAL & 3.15 & 3.98 & 5.01 \\
\hline Campbelltown & 3.94 & 3.84 & 2.97 & Mid North Coast & & & \\
\hline Bankstown-Lidcombe & 2.19 & 2.85 & 4.20 & Coffs Harbour & 4.73 & 3.76 & 7.14 \\
\hline Bowral & 2.90 & 2.76 & 4.42 & Kempsey & 2.56 & 0.00 & 0.00 \\
\hline Other hospitals & 0.00 & 0.00 & 0.00 & Port Macquarie Base & 5.10 & 4.82 & 4.83 \\
\hline TOTAL & 3.82 & 4.12 & 4.55 & Other hospitals & 10.00 & 0.00 & 0.00 \\
\hline South Eastern Sydney & & & & TOTAL & 4.80 & 3.70 & 5.41 \\
\hline St. George & 6.56 & 8.21 & 7.98 & Southern NSW & & & \\
\hline Sutherland & 3.11 & 3.54 & 4.58 & Bega & 0.00 & 0.00 & 2.27 \\
\hline Royal Hospital for Women & 3.71 & 6.76 & 7.33 & Goulburn Base & 7.55 & 6.78 & 8.00 \\
\hline TOTAL & 4.58 & 6.68 & 7.01 & Moruya & 4.76 & 3.57 & 1.72 \\
\hline Illawarra Shoalhaven & & & & Queanbeyan & 1.54 & 2.99 & 3.85 \\
\hline Shoalhaven & 3.31 & 2.27 & 1.27 & Other hospitals & 3.03 & 1.64 & 5.13 \\
\hline Wollongong & 3.13 & 1.99 & 3.27 & TOTAL & 3.64 & 3.19 & 4.09 \\
\hline Other hospitals & 16.00 & 0.00 & 15.79 & Murrumbidgee & & & \\
\hline TOTAL & 3.67 & 1.99 & 3.15 & Griffith Base & 3.70 & 1.30 & 6.41 \\
\hline Western Sydney & & & & Wagga Wagga Base & 6.15 & 3.31 & 4.00 \\
\hline Auburn & 4.13 & 5.79 & 1.90 & Other hospitals & 6.04 & 4.13 & 0.87 \\
\hline Blacktown & 5.76 & 7.28 & 8.87 & TOTAL & 5.43 & 3.13 & 3.46 \\
\hline Westmead & 4.59 & 5.86 & 3.73 & Western NSW & & & \\
\hline TOTAL & 4.85 & 6.30 & 5.12 & Dubbo Base & 8.42 & 4.79 & 4.80 \\
\hline Nepean Blue Mountains & & & & Mudgee & 2.00 & 4.84 & 4.44 \\
\hline Blue Mountains & 6.45 & 5.71 & 3.23 & Bathurst Base & 0.00 & 6.84 & 5.98 \\
\hline Nepean & 4.78 & 3.69 & 5.33 & Orange Base & 7.23 & 7.74 & 8.27 \\
\hline Hawkesbury & 4.00 & 1.54 & 2.48 & Other hospitals & 6.59 & 9.68 & 6.06 \\
\hline Other hospitals & 2.94 & 2.50 & 0.00 & TOTAL & 5.94 & 6.69 & 5.93 \\
\hline TOTAL & 4.69 & 3.38 & 4.56 & Far West & & & \\
\hline Northern Sydney & & & & Broken Hill Base & 2.33 & 2.22 & 2.33 \\
\hline Hornsby & 6.56 & 8.92 & 5.39 & TOTAL & 2.33 & 2.22 & 2.33 \\
\hline Manly & 1.94 & 2.90 & 2.88 & Private Hospitals & & & \\
\hline Mona Vale & 4.65 & 4.93 & 5.88 & Mater, North Sydney & 3.30 & 3.93 & 4.09 \\
\hline Royal North Shore & 8.19 & 10.04 & 10.50 & North Shore Private & 1.46 & 0.84 & 2.25 \\
\hline Other hospitals & 0.00 & 0.00 & 3.03 & Sydney Adventist & 1.90 & 1.59 & 2.16 \\
\hline TOTAL & 6.00 & 7.30 & 6.94 & North Gosford Private & 2.76 & 0.00 & 2.44 \\
\hline Central Coast & & & & Hurstville Private & 1.17 & 3.05 & 4.17 \\
\hline Gosford & 7.94 & 6.31 & 7.04 & Kareena Private & 1.23 & 0.85 & 3.08 \\
\hline Wyong & 1.56 & 0.00 & 0.00 & St. George Private & 2.84 & 3.11 & 4.61 \\
\hline TOTAL & 7.07 & 5.97 & 6.45 & Prince of Wales Private & 1.47 & 1.93 & 1.19 \\
\hline Hunter New England & & & & Norwest Private & 4.05 & 3.74 & 3.53 \\
\hline Armidale & 0.00 & 3.30 & 0.00 & Sydney Southwest Private & 3.75 & 1.93 & 1.60 \\
\hline Inverell & 0.00 & 0.00 & 0.00 & Nepean Private & 1.79 & 2.63 & 1.52 \\
\hline Tamworth Base & 5.17 & 6.35 & 4.60 & Westmead Private & 0.91 & 0.57 & 0.17 \\
\hline Manning Base & 5.26 & 1.72 & 2.46 & Figtree Private & 0.45 & 1.02 & 0.51 \\
\hline Maitland & 4.35 & 10.31 & 10.04 & Newcastle Private & 2.20 & 2.72 & 1.97 \\
\hline John Hunter & 7.85 & 10.14 & 7.80 & Calvary, Wagga Wagga & 5.94 & 4.50 & 7.81 \\
\hline Other hospitals & 3.08 & 4.62 & 2.33 & Other hospitals & 0.00 & 0.00 & 3.70 \\
\hline TOTAL & 5.49 & 7.86 & 6.23 & TOTAL & 2.17 & 2.09 & 2.43 \\
\hline & & & & TOTAL NSW* & 4.48 & 4.73 & 4.75 \\
\hline
\end{tabular}


Major perineal tears and surgical repair of the perineum.

\section{Indicator 3.6}

Definition: Total number of selected primipara undergoing surgical repair of the perineum for fourth degree tear as a percentage of the total number of selected primipara delivering vaginally.

A selected primipara is defined as a woman who is 20-34 years of age at the time of giving birth; giving birth for the first time at greater than 20 weeks gestation; singleton pregnancy; cephalic presentation; and at $37^{\circ}$ to $41^{\circ}$ weeks gestation.

Table 64 shows aggregate information for hospitals where at least 200 mothers gave birth in 2009, totals for hospitals within each Local Health District, and the NSW total.

Table 64. 3.6 Selected primipara undergoing surgical repair of the perineum for fourth degree tear by Local Health District and hospital, NSW 2007-2009\#

\begin{tabular}{|c|c|c|c|c|c|c|c|}
\hline Local Health District-hospital & $\begin{array}{c}2007 \\
\%\end{array}$ & $\begin{array}{c}2008 \\
\%\end{array}$ & $\begin{array}{c}2009 \\
\%\end{array}$ & Local Health District-hospital & $\begin{array}{c}2007 \\
\%\end{array}$ & $\begin{array}{c}2008 \\
\%\end{array}$ & $\begin{array}{c}2009 \\
\%\end{array}$ \\
\hline Sydney & & & & Northern NSW & & & \\
\hline Canterbury & 0.75 & 1.23 & 0.00 & Grafton Base & 2.22 & 0.00 & 0.00 \\
\hline Royal Prince Alfred & 0.22 & 0.07 & 0.23 & Lismore Base & 0.00 & 0.42 & 0.42 \\
\hline TOTAL & 0.34 & 0.34 & 0.17 & Murwillumbah & 0.00 & 0.00 & 0.00 \\
\hline South Western Sydney & & & & Tweed Heads & 0.33 & 0.00 & 0.00 \\
\hline Fairfield & 0.21 & 0.00 & 0.19 & Other hospitals & 0.00 & 0.00 & 0.00 \\
\hline Liverpool & 0.33 & 0.48 & 0.84 & TOTAL & 0.39 & 0.13 & 0.13 \\
\hline Campbelltown & 0.70 & 0.35 & 0.00 & Mid North Coast & & & \\
\hline Bankstown-Lidcombe & 0.22 & 0.19 & 0.36 & Coffs Harbour & 0.00 & 0.00 & 0.48 \\
\hline Bowral & 0.00 & 0.69 & 0.00 & Kempsey & 0.00 & 0.00 & 0.00 \\
\hline Other hospitals & 0.00 & 0.00 & 0.00 & Port Macquarie Base & 0.00 & 0.00 & 0.69 \\
\hline TOTAL & 0.33 & 0.30 & 0.35 & Other hospitals & 0.00 & 0.00 & 0.00 \\
\hline South Eastern Sydney & & & & TOTAL & 0.00 & 0.00 & 0.49 \\
\hline St. George & 0.84 & 0.47 & 0.78 & Southern NSW & & & \\
\hline Sutherland & 0.31 & 0.29 & 0.00 & Bega & 0.00 & 0.00 & 0.00 \\
\hline Royal Hospital for Women & 0.28 & 0.39 & 0.20 & Goulburn Base & 0.00 & 0.00 & 0.00 \\
\hline TOTAL & 0.47 & 0.40 & 0.34 & Moruya & 0.00 & 0.00 & 0.00 \\
\hline Illawarra Shoalhaven & & & & Queanbeyan & 0.00 & 1.49 & 0.00 \\
\hline Shoalhaven & 0.83 & 0.00 & 0.63 & Other hospitals & 0.00 & 0.00 & 0.00 \\
\hline Wollongong & 0.42 & 0.20 & 0.41 & TOTAL & 0.00 & 0.35 & 0.00 \\
\hline Other hospitals & 4.00 & 5.26 & 0.00 & Murrumbidgee & & & \\
\hline TOTAL & 0.64 & 0.31 & 0.45 & Griffith Base & 0.93 & 0.00 & 0.00 \\
\hline Western Sydney & & & & Wagga Wagga Base & 0.00 & 0.83 & 0.00 \\
\hline Auburn & 0.29 & 0.00 & 1.27 & Other hospitals & 0.67 & 0.00 & 0.87 \\
\hline Blacktown & 0.35 & 0.15 & 0.14 & TOTAL & 0.52 & 0.31 & 0.31 \\
\hline Westmead & 0.29 & 0.18 & 0.69 & Western NSW & & & \\
\hline TOTAL & 0.31 & 0.15 & 0.60 & Dubbo Base & 0.00 & 0.00 & 0.00 \\
\hline Nepean Blue Mountains & & & & Mudgee & 0.00 & 1.61 & 0.00 \\
\hline Blue Mountains & 0.00 & 0.00 & 0.00 & Bathurst Base & 1.03 & 0.00 & 0.00 \\
\hline Nepean & 0.64 & 0.48 & 0.48 & Orange Base & 0.60 & 0.00 & 0.00 \\
\hline Hawkesbury & 0.00 & 0.00 & 0.00 & Other hospitals & 0.00 & 0.00 & 0.00 \\
\hline Other hospitals & 0.00 & 0.00 & 0.00 & TOTAL & 0.33 & 0.16 & 0.00 \\
\hline TOTAL & 0.46 & 0.36 & 0.36 & Far West & & & \\
\hline Northern Sydney & & & & Broken Hill Base & 0.00 & 0.00 & 0.00 \\
\hline Hornsby & 0.33 & 0.00 & 0.00 & TOTAL & 0.00 & 0.00 & 0.00 \\
\hline Manly & 0.00 & 0.41 & 0.72 & Private Hospitals & & & \\
\hline Mona Vale & 0.58 & 0.00 & 0.00 & Mater, North Sydney & 0.66 & 0.00 & 0.23 \\
\hline Royal North Shore & 0.87 & 0.64 & 0.20 & North Shore Private & 0.21 & 0.00 & 0.45 \\
\hline Other hospitals & 0.00 & 0.00 & 0.00 & Sydney Adventist & 0.24 & 0.23 & 0.22 \\
\hline TOTAL & 0.52 & 0.34 & 0.25 & North Gosford Private & 0.00 & 0.00 & 0.00 \\
\hline Central Coast & & & & Hurstville Private & 0.58 & 0.00 & 0.00 \\
\hline Gosford & 0.50 & 0.47 & 0.00 & Kareena Private & 0.00 & 0.00 & 0.00 \\
\hline Wyong & 1.56 & 0.00 & 0.00 & St. George Private & 1.29 & 0.78 & 0.58 \\
\hline TOTAL & 0.64 & 0.44 & 0.00 & Prince of Wales Private & 0.24 & 0.00 & 0.00 \\
\hline Hunter New England & & & & Norwest Private & 0.00 & 0.00 & 0.39 \\
\hline Armidale & 0.00 & 0.00 & 0.00 & Sydney Southwest Private & 0.00 & 0.77 & 0.00 \\
\hline Inverell & 0.00 & 0.00 & 0.00 & Nepean Private & 0.00 & 0.00 & 0.00 \\
\hline Tamworth Base & 0.00 & 0.00 & 1.15 & Westmead Private & 0.37 & 0.00 & 0.17 \\
\hline Manning Base & 0.00 & 0.00 & 0.00 & Figtree Private & 0.00 & 0.00 & 0.00 \\
\hline Maitland & 0.67 & 0.00 & 0.36 & Newcastle Private & 0.00 & 0.25 & 1.23 \\
\hline John Hunter & 0.70 & 0.14 & 0.67 & Calvary, Wagga Wagga & 0.00 & 0.90 & 3.13 \\
\hline Other hospitals & 0.00 & 0.00 & 0.00 & Other hospitals & 2.17 & 0.00 & 1.85 \\
\hline \multirow[t]{2}{*}{ TOTAL } & 0.42 & 0.06 & 0.49 & TOTAL & 0.33 & 0.17 & 0.39 \\
\hline & & & & TOTAL NSW* & 0.38 & 0.24 & 0.35 \\
\hline
\end{tabular}




\section{General anaesthesia for caesarean section.}

\section{Indicator 4.1}

Definition: Total number of women having a general anaesthetic for a caesarean section as a percentage of women having a caesarean section
Table 65 shows aggregate information for hospitals where at least 200 mothers gave birth in 2009, totals for hospitals within each Local Health District, and the NSW total.

Table 65. 4.1 General anaesthesia for caesarean section by Local Health District and hospital, NSW 2007-2009\#

\begin{tabular}{|c|c|c|c|c|c|c|c|}
\hline Local Health District-hospital & $\begin{array}{c}2007 \\
\%\end{array}$ & $\begin{array}{c}2008 \\
\%\end{array}$ & $\begin{array}{c}2009 \\
\%\end{array}$ & Local Health District-hospital & $\begin{array}{c}2007 \\
\%\end{array}$ & $\begin{array}{c}2008 \\
\%\end{array}$ & $\begin{array}{c}2009 \\
\%\end{array}$ \\
\hline Sydney & & & & Northern NSW & & & \\
\hline Canterbury & 13.28 & 14.09 & 14.06 & Grafton Base & 26.67 & 29.25 & 15.83 \\
\hline Royal Prince Alfred & 12.25 & 12.19 & 11.89 & Lismore Base & 4.86 & 6.49 & 6.43 \\
\hline TOTAL & 12.45 & 12.53 & 12.36 & Murwillumbah & 6.03 & 8.33 & 3.70 \\
\hline South Western Sydney & & & & Tweed Heads & 11.15 & 10.76 & 10.31 \\
\hline Fairfield & 50.26 & 43.49 & 36.19 & Other hospitals & 0.00 & 0.00 & 0.00 \\
\hline Liverpool & 21.65 & 20.22 & 18.22 & TOTAL & 10.12 & 11.51 & 9.19 \\
\hline Campbelltown & 11.73 & 12.82 & 16.10 & Mid North Coast & & & \\
\hline Bankstown-Lidcombe & 22.67 & 20.09 & 15.30 & Coffs Harbour & 11.67 & 14.92 & 9.12 \\
\hline Bowral & 15.67 & 14.71 & 9.35 & Kempsey & 8.77 & 10.45 & 16.33 \\
\hline Other hospitals & 23.51 & 21.72 & 19.27 & Port Macquarie Base & 10.82 & 8.00 & 10.20 \\
\hline TOTAL & 0.33 & 0.30 & 0.35 & Other hospitals & 0.00 & 0.0 & 0.0 \\
\hline South Eastern Sydney & & & & TOTAL & 10.98 & 11.75 & 10.14 \\
\hline St. George & 11.32 & 8.29 & 8.59 & Southern NSW & & & \\
\hline Sutherland & 6.37 & 8.33 & 10.11 & Bega & 22.86 & 8.33 & 15.09 \\
\hline Royal Hospital for Women & 4.05 & 4.61 & 4.73 & Goulburn Base & 29.27 & 37.04 & 24.36 \\
\hline TOTAL & 6.39 & 6.05 & 6.49 & Moruya & 23.17 & 18.68 & 17.20 \\
\hline Illawarra Shoalhaven & & & & Queanbeyan & 10.71 & 18.18 & 16.36 \\
\hline Shoalhaven & 11.22 & 6.93 & 8.21 & Other hospitals & 5.80 & 9.23 & 4.88 \\
\hline Wollongong & 12.57 & 9.75 & 10.59 & TOTAL & 18.83 & 19.76 & 16.88 \\
\hline Other hospitals & 12.50 & 8.70 & 8.33 & Murrumbidgee & & & \\
\hline TOTAL & 12.08 & 8.78 & 9.78 & Griffith Base & 18.63 & 18.42 & 12.15 \\
\hline Western Sydney & & & & Wagga Wagga Base & 12.74 & 13.40 & 5.68 \\
\hline Auburn & 27.34 & 19.48 & 15.21 & Other hospitals & 13.36 & 7.01 & 10.95 \\
\hline Blacktown & 17.57 & 18.88 & 13.03 & TOTAL & 14.12 & 11.92 & 8.94 \\
\hline Westmead & 19.04 & 18.31 & 14.69 & Western NSW & & & \\
\hline TOTAL & 19.47 & 18.62 & 14.19 & Dubbo Base & 25.89 & 24.08 & 20.77 \\
\hline Nepean Blue Mountains & & & & Mudgee & 25.42 & 15.00 & 10.42 \\
\hline Blue Mountains & 8.06 & 5.08 & 10.67 & Bathurst Base & 10.92 & 12.27 & 14.97 \\
\hline Nepean & 23.18 & 20.32 & 19.98 & Orange Base & 12.87 & 12.75 & 16.96 \\
\hline Hawkesbury & 10.64 & 11.59 & 14.39 & Other hospitals & 28.13 & 23.44 & 24.63 \\
\hline Other hospitals & 10.14 & 7.59 & 3.33 & TOTAL & 19.45 & 18.09 & 18.55 \\
\hline TOTAL & 20.05 & 17.69 & 17.81 & Far West & & & \\
\hline Northern Sydney & & & & Broken Hill Base & 18.67 & 11.49 & 13.10 \\
\hline Hornsby & 9.06 & 8.11 & 7.35 & TOTAL & 18.67 & 11.49 & 13.10 \\
\hline Manly & 8.65 & 7.78 & 6.13 & Private Hospitals & & & \\
\hline Mona Vale & 6.39 & 6.64 & 6.90 & Mater, North Sydney & 4.33 & 3.36 & 2.85 \\
\hline Royal North Shore & 7.82 & 8.43 & 8.90 & North Shore Private & 2.48 & 3.62 & 2.73 \\
\hline Other hospitals & 8.00 & 8.00 & 7.84 & Sydney Adventist & 5.98 & 6.77 & 3.77 \\
\hline TOTAL & 0.52 & 0.34 & 0.25 & North Gosford Private & 5.57 & 5.29 & 18.88 \\
\hline Central Coast & & & & Hurstville Private & 4.49 & 3.51 & 3.90 \\
\hline Gosford & 14.07 & 13.88 & 11.06 & Kareena Private & 4.85 & 1.77 & 2.60 \\
\hline Wyong & 55.56 & 100.00 & 100.00 & St. George Private & 4.33 & 5.11 & 4.87 \\
\hline TOTAL & 14.50 & 13.98 & 11.16 & Prince of Wales Private & 0.47 & 3.28 & 25.46 \\
\hline Hunter New England & & & & Norwest Private & 8.53 & 10.25 & 12.57 \\
\hline Armidale & 4.80 & 8.47 & 10.19 & Sydney Southwest Private & 10.72 & 5.62 & 4.53 \\
\hline Inverell & 4.40 & 1.96 & 1.25 & Nepean Private & 7.57 & 6.07 & 6.11 \\
\hline Tamworth Base & 13.91 & 17.67 & 16.04 & Westmead Private & 9.03 & 6.60 & 6.30 \\
\hline Manning Base & 23.57 & 19.32 & 22.11 & Figtree Private & 7.94 & 2.56 & 5.29 \\
\hline Maitland & 11.68 & 14.53 & 12.27 & Newcastle Private & 6.57 & 4.46 & 3.03 \\
\hline John Hunter & 20.59 & 17.83 & 19.27 & Calvary, Wagga Wagga & 2.55 & 3.53 & 5.02 \\
\hline Other hospitals & 20.21 & 21.88 & 26.96 & Other hospitals & 11.90 & 9.62 & 10.34 \\
\hline \multirow[t]{2}{*}{ TOTAL } & 16.61 & 16.43 & 17.41 & TOTAL & 5.25 & 4.76 & 7.49 \\
\hline & & & & TOTAL NSW* & 11.91 & 11.19 & 11.40 \\
\hline
\end{tabular}




\section{Postpartum haemorrhage and blood transfusion after vaginal birth. \\ Indicator 7.1}

Definition: Total number of women who give birth vaginally who require a blood transfusion during the same admission as a percentage of women who give birth vaginally.
Table 66 shows aggregate information for hospitals where at least 200 mothers gave birth in 2009, totals for hospitals within each Local Health District, and the NSW total.

Table 66. 7.1 Postpartum haemorrhage and blood transfusion by Local Health District and hospital, NSW 2007-2009\#

\begin{tabular}{|c|c|c|c|c|c|c|c|}
\hline Local Health District-hospital & $\begin{array}{c}2007 \\
\%\end{array}$ & $\begin{array}{c}2008 \\
\%\end{array}$ & $\begin{array}{c}2009 \\
\%\end{array}$ & Local Health District-hospital & $\begin{array}{c}2007 \\
\%\end{array}$ & $\begin{array}{c}2008 \\
\%\end{array}$ & $\begin{array}{c}2009 \\
\%\end{array}$ \\
\hline Sydney & & & & Northern NSW & & & \\
\hline Canterbury & 0.08 & 0.08 & 0.24 & Grafton Base & 2.40 & 2.45 & 3.68 \\
\hline Royal Prince Alfred & 0.11 & 0.11 & 0.17 & Lismore Base & 3.94 & 2.42 & 3.28 \\
\hline TOTAL & 0.10 & 0.10 & 0.19 & Murwillumbah & 1.90 & 0.73 & 0.65 \\
\hline South Western Sydney & & & & Tweed Heads & 4.70 & 2.65 & 3.43 \\
\hline Fairfield & 0.12 & 0.49 & 0.39 & Other hospitals & 4.80 & 3.41 & 3.03 \\
\hline Liverpool & 0.30 & 0.43 & 0.26 & TOTAL & 3.86 & 2.41 & 3.23 \\
\hline Campbelltown & 0.20 & 0.19 & 0.25 & Mid North Coast & & & \\
\hline Bankstown-Lidcombe & 0.17 & 0.91 & 0.58 & Coffs Harbour & 2.49 & 3.00 & 2.07 \\
\hline Bowral & 0.36 & 0.19 & 0.94 & Kempsey & 3.45 & 1.66 & 3.11 \\
\hline Other hospitals & 0.00 & 0.00 & 0.00 & Port Macquarie Base & 2.03 & 2.11 & 2.21 \\
\hline TOTAL & 0.22 & 0.47 & 0.39 & Other hospitals & 4.49 & 2.38 & 3.92 \\
\hline South Eastern Sydney & & & & TOTAL & 2.57 & 2.46 & 2.34 \\
\hline St. George & 0.15 & 0.33 & 0.32 & Southern NSW & & & \\
\hline Sutherland & 0.22 & 0.32 & 0.40 & Bega & 6.19 & 7.10 & 3.95 \\
\hline Royal Hospital for Women & 1.03 & 0.74 & 0.79 & Goulburn Base & 2.38 & 3.24 & 2.68 \\
\hline TOTAL & 0.59 & 0.54 & 0.56 & Moruya & 4.15 & 4.01 & 3.88 \\
\hline Illawarra Shoalhaven & & & & Queanbeyan & 6.38 & 3.51 & 5.91 \\
\hline Shoalhaven & 0.19 & 0.36 & 0.33 & Other hospitals & 2.30 & 3.57 & 4.58 \\
\hline Wollongong & 0.87 & 0.37 & 0.67 & TOTAL & 4.03 & 4.10 & 4.22 \\
\hline Other hospitals & 0.97 & 0.00 & 0.00 & Murrumbidgee & & & \\
\hline TOTAL & 0.73 & 0.35 & 0.57 & Griffith Base & 5.98 & 4.90 & 7.26 \\
\hline Western Sydney & & & & Wagga Wagga Base & 8.61 & 3.17 & 1.52 \\
\hline Auburn & 0.17 & 0.35 & 0.74 & Other hospitals & 6.38 & 2.32 & 3.93 \\
\hline Blacktown & 0.48 & 0.45 & 0.38 & TOTAL & 7.11 & 3.18 & 3.75 \\
\hline Westmead & 0.13 & 0.48 & 0.34 & Western NSW & & & \\
\hline TOTAL & 0.26 & 0.45 & 0.42 & Dubbo Base & 3.42 & 2.93 & 2.19 \\
\hline Nepean Blue Mountains & & & & Mudgee & 1.04 & 1.10 & 3.35 \\
\hline Blue Mountains & 1.79 & 0.00 & 1.76 & Bathurst Base & 5.68 & 4.45 & 4.58 \\
\hline Nepean & 0.43 & 0.44 & 0.44 & Orange Base & 2.19 & 3.92 & 1.84 \\
\hline Hawkesbury & 0.66 & 0.17 & 0.51 & Other hospitals & 4.43 & 3.34 & 3.42 \\
\hline Other hospitals & 2.47 & 1.96 & 3.88 & TOTAL & 3.45 & 3.38 & 2.77 \\
\hline TOTAL & 0.65 & 0.45 & 0.65 & Far West & & & \\
\hline Northern Sydney & & & & Broken Hill Base & 4.10 & 7.53 & 4.55 \\
\hline Hornsby & 0.56 & 0.11 & 1.30 & TOTAL & 4.10 & 7.53 & 4.55 \\
\hline Manly & 0.00 & 0.29 & 0.74 & Private Hospitals & & & \\
\hline Mona Vale & 0.56 & 0.43 & 0.00 & Mater, North Sydney & 2.72 & 2.74 & 1.92 \\
\hline Royal North Shore & 0.26 & 0.39 & 0.61 & North Shore Private & 0.74 & 0.14 & 0.21 \\
\hline Other hospitals & 0.00 & 0.83 & 0.00 & Sydney Adventist & 1.60 & 3.22 & 3.65 \\
\hline TOTAL & 0.32 & 0.32 & 0.75 & North Gosford Private & 3.73 & 1.85 & 2.70 \\
\hline Central Coast & & & & Hurstville Private & 1.43 & 1.49 & 1.63 \\
\hline Gosford & 0.48 & 0.45 & 0.49 & Kareena Private & 0.00 & 1.74 & 0.53 \\
\hline Wyong & 0.39 & 0.68 & 0.43 & St. George Private & 0.36 & 0.27 & 0.70 \\
\hline TOTAL & 0.47 & 0.47 & 0.48 & Prince of Wales Private & 2.68 & 2.52 & 2.12 \\
\hline Hunter New England & & & & Norwest Private & 1.41 & 1.10 & 1.36 \\
\hline Armidale & 2.13 & 1.77 & 0.30 & Sydney Southwest Private & 1.62 & 1.84 & 0.69 \\
\hline Inverell & 0.56 & 1.89 & 0.57 & Nepean Private & 3.64 & 3.28 & 1.40 \\
\hline Tamworth Base & 3.49 & 1.50 & 0.80 & Westmead Private & 0.36 & 0.43 & 0.46 \\
\hline Manning Base & 2.66 & 0.92 & 0.72 & Figtree Private & 1.81 & 1.28 & 0.16 \\
\hline Maitland & 0.34 & 0.34 & 0.09 & Newcastle Private & 0.53 & 0.25 & 0.33 \\
\hline John Hunter & 0.55 & 0.38 & 0.54 & Calvary, Wagga Wagga & 1.91 & 2.17 & 1.55 \\
\hline Other hospitals & 1.78 & 0.78 & 0.58 & Other hospitals & 2.40 & 2.00 & 0.00 \\
\hline \multirow[t]{2}{*}{ TOTAL } & 1.24 & 0.68 & 0.50 & TOTAL & 1.51 & 1.50 & 1.29 \\
\hline & & & & TOTAL NSW* & 1.23 & 1.05 & 1.04 \\
\hline
\end{tabular}




\section{Intrauterine growth restriction.}

\section{Indicator 8.1}

Definition: Total number of deliveries with birth weight less than 2750 grams at $40^{\circ}$ weeks gestation or beyond as a percentage of the total number of deliveries at $40^{\circ}$ weeks gestation or beyond.
Table 67 shows aggregate information for hospitals where at least 200 babies were born in 2009, totals for hospitals within each Local Health District, and the NSW total.

Table 67. 8.1 Intrauterine growth restriction at 40 weeks gestation or more by Local Health District and hospital, NSW 2007-2009\#

\begin{tabular}{|c|c|c|c|c|c|c|c|}
\hline Local Health District-hospital & $\begin{array}{c}2007 \\
\%\end{array}$ & $\begin{array}{c}2008 \\
\%\end{array}$ & $\begin{array}{c}2009 \\
\%\end{array}$ & Local Health District-hospital & $\begin{array}{c}2007 \\
\%\end{array}$ & $\begin{array}{c}2008 \\
\%\end{array}$ & $\begin{array}{c}2009 \\
\%\end{array}$ \\
\hline Sydney & & & & Northern NSW & & & \\
\hline Canterbury & 2.59 & 2.21 & 2.04 & Grafton Base & 1.08 & 1.97 & 1.19 \\
\hline Royal Prince Alfred & 1.13 & 1.75 & 1.38 & Lismore Base & 1.73 & 1.41 & 1.39 \\
\hline TOTAL & 1.49 & 1.86 & 1.55 & Murwillumbah & 1.79 & 1.81 & 4.95 \\
\hline South Western Sydney & & & & Tweed Heads & 0.77 & 1.38 & 1.51 \\
\hline Fairfield & 2.01 & 2.77 & 2.12 & Other hospitals & 3.01 & 2.56 & 1.32 \\
\hline Liverpool & 2.87 & 2.33 & 2.20 & TOTAL & 1.44 & 1.61 & 1.58 \\
\hline Campbelltown & 2.16 & 1.14 & 1.41 & Mid North Coast & & & \\
\hline Bankstown-Lidcombe & 1.69 & 1.11 & 2.71 & Coffs Harbour & 1.89 & 1.56 & 0.89 \\
\hline Bowral & 2.26 & 1.36 & 1.67 & Kempsey & 3.76 & 2.27 & 2.63 \\
\hline Other hospitals & 0.00 & 0.00 & 0.00 & Port Macquarie Base & 2.10 & 2.84 & 2.12 \\
\hline TOTAL & 2.23 & 1.77 & 2.04 & Other hospitals & 4.26 & 0.00 & 3.13 \\
\hline South Eastern Sydney & & & & TOTAL & 2.39 & 2.04 & 1.63 \\
\hline St. George & 2.10 & 2.07 & 2.01 & Southern NSW & & & \\
\hline Sutherland & 1.80 & 1.12 & 0.83 & Bega & 0.00 & 2.56 & 0.64 \\
\hline Royal Hospital for Women & 1.49 & 0.78 & 1.39 & Goulburn Base & 4.19 & 5.88 & 1.79 \\
\hline TOTAL & 1.74 & 1.24 & 1.48 & Moruya & 1.63 & 3.24 & 2.96 \\
\hline Illawarra Shoalhaven & & & & Queanbeyan & 2.58 & 2.61 & 2.66 \\
\hline Shoalhaven & 2.28 & 1.83 & 1.01 & Other hospitals & 2.44 & 2.82 & 2.73 \\
\hline Wollongong & 1.63 & 1.81 & 1.12 & TOTAL & 2.44 & 3.58 & 2.15 \\
\hline Other hospitals & 3.57 & 0.00 & 0.00 & Murrumbidgee & & & \\
\hline TOTAL & 1.87 & 1.76 & 1.04 & Griffith Base & 3.33 & 1.35 & 2.29 \\
\hline Western Sydney & & & & Wagga Wagga Base & 3.10 & 1.41 & 1.65 \\
\hline Auburn & 1.98 & 1.90 & 2.04 & Other hospitals & 2.06 & 2.39 & 1.71 \\
\hline Blacktown & 1.97 & 2.06 & 1.80 & TOTAL & 2.70 & 1.83 & 1.81 \\
\hline Westmead & 2.16 & 3.52 & 2.75 & Western NSW & & & \\
\hline TOTAL & 2.06 & 2.72 & 2.30 & Dubbo Base & 3.03 & 2.28 & 2.31 \\
\hline Nepean Blue Mountains & & & & Mudgee & 1.95 & 3.36 & 1.47 \\
\hline Blue Mountains & 0.62 & 1.85 & 0.00 & Bathurst Base & 3.34 & 4.82 & 2.73 \\
\hline Nepean & 1.53 & 1.55 & 1.34 & Orange Base & 2.40 & 1.09 & 2.10 \\
\hline Hawkesbury & 1.39 & 0.85 & 1.10 & Other hospitals & 1.69 & 1.55 & 3.90 \\
\hline Other hospitals & 3.30 & 2.35 & 5.80 & TOTAL & 2.59 & 2.47 & 2.56 \\
\hline TOTAL & 1.52 & 1.48 & 1.37 & Far West & & & \\
\hline Northern Sydney & & & & Broken Hill Base & 2.21 & 4.69 & 6.40 \\
\hline Hornsby & 1.81 & 0.67 & 0.80 & TOTAL & 2.21 & 4.69 & 6.40 \\
\hline Manly & 1.00 & 0.42 & 0.51 & Private Hospitals & & & \\
\hline Mona Vale & 2.02 & 0.65 & 0.68 & Mater, North Sydney & 1.59 & 1.29 & 0.85 \\
\hline Royal North Shore & 1.70 & 2.38 & 1.62 & North Shore Private & 1.32 & 1.64 & 2.30 \\
\hline Other hospitals & 2.22 & 1.45 & 0.00 & Sydney Adventist & 0.68 & 1.14 & 2.25 \\
\hline TOTAL & 1.64 & 1.25 & 1.01 & North Gosford Private & 2.45 & 0.36 & 0.76 \\
\hline Central Coast & & & & Hurstville Private & 1.52 & 0.95 & 1.38 \\
\hline Gosford & 1.51 & 0.85 & 1.00 & Kareena Private & 0.70 & 1.28 & 1.72 \\
\hline Wyong & 0.66 & 0.00 & 1.34 & St. George Private & 2.03 & 1.69 & 1.63 \\
\hline TOTAL & 1.41 & 0.79 & 1.04 & Prince of Wales Private & 2.28 & 1.22 & 1.30 \\
\hline Hunter New England & & & & Norwest Private & 1.21 & 1.96 & 2.37 \\
\hline Armidale & 5.22 & 0.97 & 2.35 & Sydney Southwest Private & 0.95 & 1.00 & 1.24 \\
\hline Inverell & 5.68 & 1.45 & 5.08 & Nepean Private & 0.64 & 1.27 & 1.67 \\
\hline Tamworth Base & 2.26 & 2.23 & 2.71 & Westmead Private & 2.46 & 2.26 & 1.60 \\
\hline Manning Base & 1.31 & 3.08 & 2.48 & Figtree Private & 1.18 & 1.17 & 0.41 \\
\hline Maitland & 0.68 & 2.37 & 1.62 & Newcastle Private & 0.81 & 1.47 & 1.63 \\
\hline John Hunter & 2.65 & 2.19 & 1.77 & Calvary, Wagga Wagga & 0.76 & 1.61 & 1.63 \\
\hline Other hospitals & 2.95 & 1.27 & 1.42 & Other hospitals & 1.55 & 0.00 & 0.99 \\
\hline \multirow{2}{*}{ TOTAL } & 2.42 & 2.06 & 1.90 & TOTAL & 1.45 & 1.41 & 1.60 \\
\hline & & & & TOTAL NSW* & 1.87 & 1.78 & 1.72 \\
\hline
\end{tabular}




\section{Apgar score.}

\section{Indicator 9.1}

Definition: Total number of term babies born with an Apgar score of less than 7 at 5 minutes post delivery as a percentage of the total number of term babies born.

Table 68 shows aggregate information for hospitals where at least 200 babies were born in 2009, totals for hospitals within each Local Health District, and the NSW total.

Table 68. 9.1 Apgar score of 7 or less in term babies by Local Health District and hospital, NSW 2007-2009\#

\begin{tabular}{|c|c|c|c|c|c|c|c|}
\hline Local Health District-hospital & $\begin{array}{c}2007 \\
\%\end{array}$ & $\begin{array}{c}2008 \\
\%\end{array}$ & $\begin{array}{c}2009 \\
\%\end{array}$ & Local Health District-hospital & $\begin{array}{c}2007 \\
\%\end{array}$ & $\begin{array}{c}2008 \\
\%\end{array}$ & $\begin{array}{c}2009 \\
\%\end{array}$ \\
\hline Sydney & & & & Northern NSW & & & \\
\hline Canterbury & 0.78 & 0.85 & 0.86 & Grafton Base & 1.25 & 2.24 & 0.43 \\
\hline Royal Prince Alfred & 1.09 & 1.57 & 1.64 & Lismore Base & 1.85 & 0.86 & 1.14 \\
\hline TOTAL & 1.02 & 1.40 & 1.43 & Murwillumbah & 0.82 & 0.83 & 0.00 \\
\hline South Western Sydney & & & & Tweed Heads & 0.43 & 0.42 & 1.28 \\
\hline Fairfield & 1.24 & 1.32 & 2.01 & Other hospitals & 0.88 & 0.99 & 0.00 \\
\hline Liverpool & 1.10 & 0.84 & 0.79 & TOTAL & 1.12 & 0.89 & 0.97 \\
\hline Campbelltown & 1.07 & 0.92 & 0.87 & Mid North Coast & & & \\
\hline Bankstown-Lidcombe & 0.84 & 0.82 & 0.68 & Coffs Harbour & 0.94 & 0.80 & 0.59 \\
\hline Bowral & 0.75 & 1.91 & 0.91 & Kempsey & 2.80 & 2.75 & 0.69 \\
\hline Other hospitals & 0.00 & 0.00 & 0.00 & Port Macquarie Base & 2.46 & 2.13 & 2.31 \\
\hline TOTAL & 1.04 & 1.02 & 1.02 & Other hospitals & 1.19 & 0.00 & 2.04 \\
\hline South Eastern Sydney & & & & TOTAL & 1.79 & 1.52 & 1.24 \\
\hline St. George & 1.31 & 1.32 & 0.60 & Southern NSW & & & \\
\hline Sutherland & 0.54 & 0.82 & 0.82 & Bega & 0.00 & 0.52 & 0.37 \\
\hline Royal Hospital for Women & 1.27 & 1.21 & 1.50 & Goulburn Base & 1.07 & 0.70 & 0.69 \\
\hline TOTAL & 1.17 & 1.18 & 1.09 & Moruya & 1.78 & 1.45 & 1.29 \\
\hline Illawarra Shoalhaven & & & & Queanbeyan & 1.06 & 0.75 & 0.33 \\
\hline Shoalhaven & 0.78 & 0.65 & 0.86 & Other hospitals & 0.00 & 0.35 & 0.00 \\
\hline Wollongong & 0.61 & 1.02 & 1.17 & TOTAL & 0.89 & 0.80 & 0.59 \\
\hline Other hospitals & 4.69 & 2.22 & 3.41 & Murrumbidgee & & & \\
\hline TOTAL & 0.83 & 0.96 & 1.15 & Griffith Base & 1.39 & 1.78 & 1.01 \\
\hline Western Sydney & & & & Wagga Wagga Base & 1.65 & 1.22 & 1.44 \\
\hline Auburn & 0.51 & 0.53 & 1.32 & Other hospitals & 0.96 & 0.88 & 1.14 \\
\hline Blacktown & 1.10 & 1.02 & 0.76 & TOTAL & 1.30 & 1.19 & 1.22 \\
\hline Westmead & 0.97 & 1.01 & 0.90 & Western NSW & & & \\
\hline TOTAL & 0.94 & 0.94 & 0.91 & Dubbo Base & 1.81 & 1.02 & 1.51 \\
\hline Nepean Blue Mountains & & & & Mudgee & 2.03 & 0.42 & 0.90 \\
\hline Blue Mountains & 0.35 & 1.06 & 3.02 & Bathurst Base & 1.16 & 0.71 & 1.30 \\
\hline Nepean & 0.53 & 0.75 & 0.53 & Orange Base & 0.95 & 1.08 & 1.35 \\
\hline Hawkesbury & 0.99 & 0.39 & 1.46 & Other hospitals & 1.37 & 0.72 & 0.94 \\
\hline Other hospitals & 1.38 & 0.47 & 0.00 & TOTAL & 1.43 & 0.89 & 1.30 \\
\hline TOTAL & 0.64 & 0.68 & 0.81 & Far West & & & \\
\hline Northern Sydney & & & & Broken Hill Base & 1.20 & 3.19 & 0.46 \\
\hline Hornsby & 0.51 & 0.57 & 1.90 & TOTAL & 1.20 & 3.19 & 0.46 \\
\hline Manly & 1.25 & 1.00 & 1.00 & Private Hospitals & & & \\
\hline Mona Vale & 2.76 & 0.92 & 1.33 & Mater, North Sydney & 0.51 & 0.36 & 0.38 \\
\hline Royal North Shore & 0.78 & 1.00 & 1.13 & North Shore Private & 0.51 & 0.39 & 0.61 \\
\hline Other hospitals & 0.00 & 1.67 & 0.00 & Sydney Adventist & 0.82 & 0.67 & 0.23 \\
\hline TOTAL & 1.08 & 0.89 & 1.27 & North Gosford Private & 0.60 & 0.50 & 0.13 \\
\hline Central Coast & & & & Hurstville Private & 0.73 & 0.58 & 0.77 \\
\hline Gosford & 1.28 & 1.06 & 2.60 & Kareena Private & 0.19 & 1.53 & 0.63 \\
\hline Wyong & 0.00 & 1.38 & 0.85 & St. George Private & 0.69 & 0.57 & 0.58 \\
\hline TOTAL & 1.16 & 1.08 & 2.44 & Prince of Wales Private & 0.95 & 0.86 & 0.46 \\
\hline Hunter New England & & & & Norwest Private & 0.32 & 0.33 & 0.17 \\
\hline Armidale & 1.41 & 0.24 & 1.23 & Sydney Southwest Private & 0.86 & 0.84 & 0.30 \\
\hline Inverell & 0.41 & 1.24 & 0.42 & Nepean Private & 0.51 & 0.47 & 0.34 \\
\hline Tamworth Base & 0.85 & 1.42 & 1.18 & Westmead Private & 0.81 & 0.86 & 0.62 \\
\hline Manning Base & 1.34 & 1.35 & 1.01 & Figtree Private & 0.11 & 0.55 & 0.31 \\
\hline Maitland & 1.83 & 2.34 & 1.91 & Newcastle Private & 0.46 & 0.74 & 0.33 \\
\hline John Hunter & 1.77 & 1.90 & 2.39 & Calvary, Wagga Wagga & 1.48 & 0.69 & 0.51 \\
\hline Other hospitals & 1.28 & 0.67 & 0.58 & Other hospitals & 0.70 & 0.40 & 0.76 \\
\hline \multirow{2}{*}{ TOTAL } & 1.51 & 1.58 & 1.68 & TOTAL & 0.64 & 0.63 & 0.44 \\
\hline & & & & TOTAL NSW* & 1.00 & 0.99 & 1.01 \\
\hline
\end{tabular}

